\title{
Zur Theorie der windschiefen Flächen.
}

Von A. Voss in Göttingen.

Seit dem Erscheinen ron Plücker's „Neuer Geometrie des Raumes" ist bekanntlich neben die bisher ansgebildeten analytisch geometrischen Untersuchungen, deren Schwerpunkt in der Dualität zwischen Punkt und Ebene liegt, eine neue Disciplin getreten, die Liniengeometrie. So interessant und reichhaltig diejenigen Untersuchungen sind, welche sich rein mit der Theorie der Liniencomplexe und Liniencongruenzen beschäftigen, so wird man doch immer geneigt sein, denjenigen Betrachtungen einen besonderen Werth beizumessen, durch welche anf Fragen, die wir im Sinne der gewöhnlichen geometrischen Anschauungsweise aufwerfen, vermöge der Liniengeometrie ein neues licht geworfen wird. Gerade nach dieser Richtung hin scheint sich für dieselbe noch ein weites Feld zu eröffnen *). Die volle Fruchtbarkeit der liniengeometrischen Untersuchungen wird aber erst dann hervortreten, wenn man gelernt hat dieselben ganz gleichmässig im Zusammenhang mit der Punkt- und Ebenengeometrie zu verwerthen. - In den bisherigen Bestrebungen aber, welche sich auf diese dreitheilige Ausbildung der geometrischen Speculation beziehen, in der Punkt, Ebene und Gerade völlig gleichwerthige Gebilde darstellen, haben sich erst zu wenig die analytischen Schwierigkeiten überwinden lassen, welche bei der gleichzeitigen Anwendung von Punkt-, Ebenen- und Liniencoordinaten auftreten. Es erscheint daher zweckmässig, die Liniengeometrie zunächst als eine besondere Disciplin zu behandeln, und, von der ihr eigenthümlichen Auffassungsweise ausgehend, zur Punkt- und Ebenengeometrie hinüberzuleiten. Erst von in diesem Sinne angestellten liniengeometrischen Untersuchungen dürfte jener weitere Fortschritt der modernen Geometrie, den wir bezeichneten, abhängen.

*) Wir erinnexn bier insbesondere an die Untersuchungen von Herrn Klein über die Kummer'sche Fläche, ferner an die Arbeit des Herrn Sturm "das Problem der räumlichen Projectivität" (diese Annalen Bd. VI, S. 543), sowie die neuerdings erschienene des Herro Lindemann, ,Projectivische Behandlung der Mechanik starrer Körper" (diese Annalen Bd. VII, S. 56). 
Es ist daher zunächst Zweck der vorliegenden Arbeit, zu zeigen, wie sich die geometrischen Untersuchungen, die sich auf Fragen der gewöhnlichen projectivischen Auffassung beziehen, vom rein liniengeometrischen Standpunkte aus gestalten. In dieser Absicht habe ich in dem ersten Paragraphen die hauptsächlichsten Gesichtspunkte, sowie einzelne Beispiele für die rein liniengeometrische Methode in der Kürze erläutert. Sodann gehe ich dazu über, die genannte Methode auf die Theorie derjenigen windschiefen Elächen anzuwenden, welche vollständiger Schnitt dreier allgemeiner Complexe von den Graden $m$, $n, p$ sind. Diese Flächen sind bereits von Herrn Lüroth $*$ nach einigen wesentlichen Richtungen hin behandelt worden, doch bietet die hier zu Grunde gelegte Auffassung wesentlich verschiedene, zum Theil für die Theorie der windschiefen Flächen überhaupt, wie ich glaube, neue Gesichtspunkte. Dahin rechne ich insbesondere die Betrachtung der vierpunktigen Tangenten der Limienflächen, die bisher nicht in den Kreis der Untersuchungen gezogen ist. Fin besonderes Gewicht ist dabei überall auf die vollständige algebraische Behandlung der Probleme gelegt worden. - Daran knüpfe ich eine Betrachtung der windschiefen Flächen vom Geschlechte Null, deren Erzeugende sich also als rationale Functionen eines Parameters auffassen lassen, welche schliesslich zur Untersuchung solcher Flächen rom zweiten, dritten und vierten Grade verwerthet wird.

In einer demnächstigen Arbeit werde ich die liniengeometrischen Nethoden aut die Untersuchung der Complexe, ihrer Singularitäten, sowie die der singulären Elemente von Congruenzen und ihren Brennflächer anwenden. Eine kurze Nittheilung einiger Resultate derselben wurde bereits in den Göttinger Nachrichten gegeben*).

\section{I.}

Allgemeines. Methode der liniengeometrischen Untersuchung.

Im Anschluss an die Arbeiten von Herrn Klein***) bediene ich mich zur analytischen Repräsentation der Geraden der von demselben eingeführten Liniencoordinaten. Es sind dies lineare Combinationen $x_{1} x_{2} x_{3} x_{4} x_{5} x_{6}$ der sechs homogenen Plü cker-Cayley'schen Coordinaten $p_{i k}$, für welche die zwischen den letzteren bestehende Identität S. 130.

*) Lüroth, Zur Theorie der windschiefen Flächen, Crelle's Journ. Bd. 67,

*) Gött. Nachr. 1873, Juni, Juli, S. 544,611.

***) Es sei hier namentlich auf die folgenden Aufä̈tze desselben hingewiesen, auf die im Folgenden häufig Bezug genommen werden wird: Zur Theorie der Liniencomplexe ersten and zweiten Grades (Math. Ann. Bd. II, S. 198), Allgemeine lineare Transformation der Liniencoordinaten (daselbst p. 366), Ueber gewisse in der Liniengeometrie auftretende Differentialgleiehungen (Math. Ann. Bd. V, S. 287). 
in

$$
p_{12} p_{34}+p_{13} p_{42}+p_{14} p_{23}=0
$$

$$
x_{1}^{2}+x_{2}^{2}+x_{3}^{2}+x_{4}^{2}+x_{5}^{2}+x_{6}^{2}=0 \text {, }
$$

oder, wie zur Abkïraung geschrieben werden soll,

$$
\left(x^{2}\right)=0
$$

ïbergeht. Die Voraussetzung derselben in dieser kanonischen Form ist übrigens für das Folgende durchaus unwesentlich. Es würde nur einer einfachen Modification der im Folgenden entwickelten Ausdrücke bedürfen, wenn jene Identität in Gestalt einer allgemeinen quadratischen Form

$$
\sum c_{i k} x_{i} x_{k}=0
$$

angenommen wird. Die Determinante der Form $\left(x^{2}\right)$ ist 1 , dieselbe ist im Folgenden hänfig durch $\Omega$ bezeichnet, $n$ m anzudenten, dass bei Anwendung der allgemeinen Form $\Sigma c_{i k} x_{i} x_{k}$ an Stelle von $\Omega$ einfach deren Determinante

einzuführen ist.

$$
\mathbf{\Sigma} \pm\left(c_{11} c_{22} c_{33} c_{44} c_{\mathrm{s} 5} c_{66}\right)
$$

Unter Zugrundelegung der Coordinaten $x$ ist es durchaus unzweckmässig, zu den Punkt- oder Ebenencoordinaten der Raumgeometrie, aus denen die $x$ gebildet sind, wirklich zurückzugehen, wie man dies bei Anwendung der Coordinaten $p_{2 k}=x_{i} y_{k}-x_{k} y_{i}$ oft gethan hat. Es enwächst damit die Forderung einer eigenen Liniengeometrie, als deren ausschliesslich in die analytisch-geometrischen Untersuchungen direct eintretendes Gebilde (Rammelement) die Gerade zu betrachten ist. Bei dieser Auffassung, die auch Herrn Klein's Arbeiten über Liniengeometrie zu Grunde liegt*), wird die Gerade vorgestellt durch einen speciellen Complex ersten Grades, eine Fläche ist der Inbegriff ihrer Tangenten, eine Curve der ihrer sämmtlichen Treffgeraden. Curven und Flächen werden durch eine Gleichung in Liniencoordinaten, d. h. durch Complexe vorgestellt, weil sie immer durch dreifach unendlich viele Linien erzeugt werden. Diese Gleichung muss aber im ersten Falle so beschaffen sein, dass jede von den in einer Ebene liegenden Geraden gebildete Complexcurve in so viel ebene Strahlbüschel zerfällt, als der Grad der darzustellenden Curve beträgt (der Complex die fragliche Curve zur Ausnahmecurve hat), während im zweiten. Falle das Bestehen einer partiellen Differentialgleichung für alle Complexlinien erforderlich ist**). Der Punkt erscheint als Inbegriff aller Geraden, die durch ihn gehen (Strahlenbündel), die Ebene als Gesammtheit derjenigen Geraden, die in ihr liegen (Strahlennetz). Beide Gebilde enthalten nur zweifach unendlich viele Gerade, erfordern also in ihrer 
Darstellung zwei Gleichungen in Liniencoordinaten. Analytisch können wir beide Gebilde gleichzeitig darstellen als Schnitt zweier specieller linearer Complexe, deren Axen sich schneiden, d. h. durch eine zerfallende Congruenz. Fs giebt aber unendlich viele specielle lineare Complexe, denen dieselbe zerfallende Congruenz angehört, ihre Axen bilden ein ebenes Strahlbüschel. Wir werden dadurch veranlasst, die Gesammtheit dieser Axen als Ausdruck für die zerfallende Congruenz anzusehen. - Mit dieser Auffassung kornmt es nun röllig überein, wenn wir im Folgenden Punkt und Ebene gleichzeitig durch ein ebenes Strahlbüschel repräsentiren. In der That bestimmt dasselbe auf eine völlig dualistische Weise sowohl einen Punkt, das Centrum des Büschels, als eine Ebene, die Ébene desselben. Das ebene Strahlbüschel ist daher das Mittel, durch welches die liniengeometrischen Vorstellongen in Verbindung mit denen der Punkt- und Ebenepgeometrie zu bringen sind, und spielt daher eine fundamentale Rolle in denselben*).

Eine Gerade, deren Coordinaten $x_{1} x_{2} x_{3} x_{1} x_{5} x_{0}$ sind, werden wir im Folgenden symbolisch durch $x$ bezeichnen. Zwei Gerade $x, y$ sehnejden sich, wenn die Bedingung

$$
\Sigma x_{i} y_{c}=0
$$

erfüllt ist, an deren Stelle wir abkürzend

$$
(x y)=0
$$

schreiben. Cekerhaupt werden wir analog gebildete Ausdrücke wie $\Sigma A_{i} B_{i}$ immer durch $(A B)$ bezeichnen. Der Ausdruck

$$
x+\lambda y
$$

wird nur daun wieder eine Gerade vorstellen, wenn $(x+\lambda y)^{2}=0$, d. h. $(x y)=0$, dann aber gleich unendlich viele, deren Gesammtheit das ebene Strahlbüschel bildet, welches durch die sich schneidenden Geraden $x y$ bestimnt ist. Punkt und Ebene desselben bezeichnen wir durch $(x y)$. Das Doppelverhältniss von vier Strahlen des Büschels $(x y)$ hat dann den Werth

$$
x+\lambda_{1} y, \quad x+\lambda_{2} y, \quad x+\lambda_{3} y, \quad x+\lambda_{4} y
$$

$$
\frac{\lambda_{1}-\lambda_{2}}{\lambda_{1}-\lambda_{3}}: \frac{\lambda_{4}-\lambda_{2}}{\lambda_{4}-\lambda_{3}}
$$

Drei Gerade $x y z$, für welche die Beziehungen

$$
(x y)=0, \quad(y z)=0, \quad(x z)=0
$$

stattfinden, werden sich gegenseitig schneiden. Sie liegen dann ent-

*) In den erwäbriten Mittheilungen (Gött. Nachr. a. a. O.) habe ich bereits gezeigt, wie die principielle Verwendung dieses Gebildes dazu dienen kann, die Singularitäten der Brenn- und singulären Flächen von Complexen (Rückkehr-, Doppel-, algebraische Haupttangenten-, vierpunktige Berührungscurven) nach Ordnung und Classe zu bestimmen. 
weder in einer Ebene oder gehen durch einen Punkt. Welches von beiden stattfinde, würde sich allerdings vermöge des von Herrn Klein*) für deñ einen oder anderen Fall aufgestellten Determinanten-Kenmzeichens entscheiden lassen. Es bietet aber gewisse Vortheile, beide Fälle gleichzeitig zu betrachten. Jede Untersuchung, in welcher wir drei sich gegenseitig schneidende Gerade ohwe weitere Nebenbedingung einführen, wird also einen dualistischen Charalter tragen, sich zum Theil auf drei Linien durch einen Punkt, zum Theil auf drei Linien in einer Ebene beziehen. Bei einem nicht in sich dualistischen Liniengebilde werden also Abzählungen immer nur die Summe der Anzahlen der Fälle liefern, die dem einen oder anderen Verhalten entsprechen. Ist aber das ganze Gebilde, auf welches sich die Untersuchung bezieht, ein in sich duales, so muss der eine Fall ebenso oft auftreten als der andere. Die Anvendung eines besonderen Kennzeichens wird dann ïberflüssig. Dieser Umstand gestattet die liniengeometrischen Untersuchungen oft überraschend einfach. Er findet insbesondere statt in den folgenden Untersuchungen, insofern jedes vollstündige Liniengebilde, welchies durch allgemeine Complexe erzengt wird, die geforderte Dualitït besitzen muss.

Wir behandeln nach diesen allgemeinen Erörterungen zunächst einige der einfachsten Aufgaben, welche sich in der Liniengeometrie darbieten, theils weil wir der Resultate derselben in der Folge ofters benöthigt sein werden, theils um zu zeigen, wie sich auf rein liniengeometrischem Wege die Behandlung derselben gestaltet.

1) Bestimmung des Doppelverhältnisses zweier linearen Complexe. Zwei lineare Complexe geben zu einem Büschel linearer Complexe Veranlassung. Bezeichnet man durch $a, b$ die Directricen desselben, so sind zwei beliebige Complexe desselben dargestellt durch

$$
\begin{aligned}
& a_{x}+\lambda b_{x}=0, \\
& a_{x}+\mu b_{x}=0 .
\end{aligned}
$$

Eine beliebige Ebene schneidet die Directricen in zwei Punkten $A, B$ und entbält zwei Centra $c_{1} c_{2}$ ron Strablbüscheln, die den Complexen (1), (2) angehören. Die vier Punkte $A B c_{1} c_{2}$ liegen in gerader Linie und haben ein unveränderliches Doppelverhältniss, welches nach Herrn Klein als Doppelverhältniss der beiden Complexe (1), (2) bezeichnet wird. Um den Werth desselben zu bestimmen, legen wir zwei Gerade $y, z$, welche bezüglich die Directricen $a, b$ und sich unter einander schneiden, so dass

$$
(a y)=0,(b z)=0, \quad(z y)=0 .
$$

Thre Ebene stellt die schneidende Ebene dar, $y z$ bilden mit den beiden Strahlen $y+\varrho_{1} z, y+\varrho_{2} z$, welche respective (1), (2) angehören,

*) Math. Ann. Bd. II, S. 200, 206. 
vier Strahlen, deren Doppelverhältniss $\frac{\rho_{t}}{\varrho_{z}}$ das gesuchte ist. Zur Bestimmung von $\frac{Q_{1}}{Q_{z}}$ hat man die beiden Gleichungen, welche ausdrücken, dass $y+\rho_{1} z, y+o_{2} z$ Strahlen der Complexe $(1),(2)$ sind, nämlich

$$
\begin{aligned}
& (a y)+\lambda(b y)+o_{1}((a z)+\lambda(b z))=0, \\
& (a y)+\mu(b y)+e_{2}((a z)+\mu(b z))=0,
\end{aligned}
$$

aus denen folgt:

$$
\frac{a_{1}}{a_{2}}=\frac{\lambda}{\mu}
$$

Wir stellen dazu noch die folgende Betrachtung. Es seien $z_{1} z_{2} z_{3}$ drei Gerade, welche die beiden Directricen schneiden. Jeder Complex des Büschels enthält zwei Strahlen $x, y$, welche $z_{1} z_{2} z_{3}$ gleichzeitig schneiden, d. h. Erzeugende des durch $z_{1} z_{2} z_{3}$ bestimmten Hyperbolaides sind. Die vier Geraden abxy bestimmen dann auf jeder der Geraden z harmonisch liegende Punkte.

Wir beweisen dies folgendermassen. Der Complex des Büschels sei

Eine Gerade \& schneide

$$
a_{x}+\lambda b_{x}=0 \text {. }
$$

so dass

$$
a, b, z_{1} \text {, }
$$

$$
\left\{\begin{array}{rll}
(\varepsilon a)=0, & (\varepsilon b)=0, & \left(\varepsilon z_{1}\right)=0, \\
\left(z_{1} a\right)=0, & \left(z_{2} a\right)=0, & \left(z_{3} a\right)=0, \\
\left(z_{1} b\right)=0, & \left(z_{2} b\right)=0, & \left(z_{3} b\right)=0,
\end{array}\right.
$$

Wir suchen nun die Strablen $a+\rho \varepsilon$ des Büschels $(a \varepsilon)$, welche die $z_{1}$ in den Punkten treffen, in welchen sie selbst von einer Complexgeraden $x$ geschnitten wird, welche $z_{1} z_{2} z_{3}$ gleichzeitig schneidet. Man hat dann:

$$
\left\{\begin{array}{c}
\left(x z_{1}\right)=0, \quad\left(x z_{2}\right)=0, \quad\left(x z_{3}\right)=0, \\
a_{x}+\lambda b_{x}=0 \\
(a x)+\varphi(\varepsilon x)=0 .
\end{array}\right.
$$

Eliminirt man aus den 5 letzten Gleichungen und der Identität $\left(x^{2}\right)=0$ die $x$, so erhält man nach einer leichten Unformung mit Berücksichtigung der Gleichungen (3), (4) die Determinante:

$$
\mid \begin{array}{ccccc}
0 & \left(z_{1} z_{2}\right) & \left(z_{1} z_{3}\right) & 0 & \varrho\left(\varepsilon z_{1}\right) \\
\left(z_{1} z_{2}\right) & 0 & \left(z_{2} z_{3}\right) & 0 & \varrho\left(\varepsilon z_{1}\right) \\
\left(z_{4} z_{3}\right) & \left(z_{2} z_{3}\right) & 0 & 0 & \varrho\left(\varepsilon z_{3}\right) \\
0 & 0 & 0 & 2 \lambda(a b) & \lambda(a b) \\
\rho\left(\varepsilon z_{1}\right) & \rho\left(\varepsilon z_{2}\right) & \varrho\left(\varepsilon z_{3}\right) & \lambda(a b) & 0
\end{array}=0 .
$$

Dieser Ausdruck jst von der Form

$$
\alpha-\beta \rho^{2}=0 \text {. }
$$


Wir erhalten daher zwei Strahlen mit den Parametern

$$
\varrho_{1}=+\sqrt{\frac{\alpha}{\beta}}, \quad \varrho_{2}=-\sqrt{\frac{\alpha}{\beta}},
$$

welche mit $a \varepsilon$ harmonisch liegen.

Da die Directricen $a b$ conjugirte Polaren in Bezug auf den Complex $a_{x}+\lambda b_{x}=0$ sind, so kann man den folgenden Satz aussprechen:

Jede Erzeugende zweiter Art eines Hyperboloids, won welchem zwei Erzeugende erster Art conjuginte Polaren in Beaug auf einen tinearen Complex sind, wird won den beiden Complexgeraden, welche selbst Erzeugende erster Art des Hyperboloides sind, in zwei Punlten getroffen, welche harmonisch liegen al ihren Schnittpunkten mit den beiden conjugirten Polaren.

2) Betrachtung von vier windschiefen Linien a bcd. Es giebt bekanntlich zwei Gerade, welche die vier gegebenen schneiden. Die Coordinaten derselben sind gegeben durch die Gleichungen

$$
\begin{gathered}
(a x)=0, \quad(b x)=0, \quad(c x)=0, \quad(d x)=0, \\
\left(x^{2}\right)=0 .
\end{gathered}
$$

Man erhält das Product der Gleichungen der beiden speciellen linearen Complexe, welche die beiden Geraden $x$ vertreten, wenn man denselben noch die Bedingung

$$
(z x)=0
$$

hinzufügt und dann die $x$ eliminirt in Gestalt der Determinante:

$$
\begin{aligned}
& \left|\begin{array}{ll}
0 & (a b)(a c)(a d)(a z)
\end{array}\right| \\
& \text { (ab) } 0(b c)(b d)(b z) \\
& (a c)(b c) \quad 0 \quad(c d)(c z)=0 \text {. } \\
& (a d)(b d)(d c) \quad 0 \quad(d z) \\
& (a z)(b z)(c z)(d z) 0
\end{aligned}
$$

Die beiden Geraden $x$ coincidiren, wenn der Kern dieser Determinante verschwindet, $d$. h. wenn

$$
\left|\begin{array}{cccc}
0 & (a b) & (a c) & (a d) \\
(a b) & 0 & (b c) & (b d) \\
(a c) & (b c) & 0 & (c d) \\
(a d) & (b d) & (c d) & 0
\end{array}\right|=0
$$

Wir bestimmen jetzt das Doppelverhältniss der vier Punkte, in denen die beiden Geraden $x$ von $a b c d$ geschnitten werden, auf folgende Weise. Es sei $y$ eine Gerade, welche $a d x$ schneidet. Wir suchen dann die Strahlen

$$
y+\lambda_{1} a, y+\lambda_{2} a
$$

des Büschels $(y a)$, welche respective $b, c$ schneiden. Für dieselben ist 


$$
\begin{aligned}
& (y b)+\lambda_{1}(a b)=0, \\
& (y c)+\lambda_{2}(a c)=0 .
\end{aligned}
$$

Das gesuchte Doppelverhältniss ist daher:

Aus den Gleichungen

$$
\frac{h_{i}}{h_{2}}=\frac{(y b)}{(y e)}: \frac{(a b)}{(a c)}
$$

$(y x)=0, \quad(a x)=0, \quad(b x)=0, \quad(c x)=0, \quad(a x)=0, \quad\left(x^{2}\right)=0$,

erhält man aber

$$
(d y)=0, \quad(a y)=0
$$

$$
\begin{array}{ccccc}
0 & 0 & (y b) & (y c) & 0 \\
0 & 0 & (a b) & (a c) & (a d) \\
(y b) & (b a) & 0 & (b c) & (b d)=0 \\
(y c) & (a c) & (b c) & 0 & (c d) \\
0 & (a d) & (b d) & (c d) & 0
\end{array}
$$

und damit die beiden Werthe $\frac{(y b)}{(y c)}$, welche den fraglichen Doppelverhältnissen auf den beiden Geraden $\infty$ entsprechen.

Für vier Gerade mit verschwindender Determinante $(5)$ hat $\left(\frac{y l_{i}}{y c}\right)$ einen sehr einfachen Werth. Es existiren darn vier Zahlen $\mu_{1} \mu_{2} \mu_{3} \mu_{1}$, welche den Gleichungen:

$$
\left\{\begin{aligned}
\mu_{2}(a b)+\mu_{3}(a c)+\mu_{1}(a d) & =0 \\
\mu_{1}(a b)+\mu_{3}(b c)+\mu_{4}(b d) & =0 \\
\mu_{1}(a c)+\mu_{2}(b c)+\mu_{4}(c d) & =0 \\
\mu_{1}(a d)+\mu_{2}(b d)+\mu_{3}(c d) & =0
\end{aligned}\right.
$$

genügen. Alsdanr ist

$$
\mu_{2}(y b)+\mu_{3}(y c)=0
$$

Bestimmt man $\mu_{z}$ und $\mu_{3}$, so ergiebt sich

$$
\frac{\lambda_{1}}{\lambda_{2}}=\frac{(b d)(a c)-(c d)(a b)+(a d)(b e)}{(b d)(a c)-(c d)(a b)-(a d)(b c)} .
$$

Das Quadrat dieses Ausdrucks hat aber, vermöge der verschwindenden Determinante der Gleichungen (6), den Werth

$$
\frac{(a c)}{(a b)}: \frac{(d c)}{(d b)}
$$

so dass der absolute Werth des Doppelverhältnisses

ist*).

$$
\frac{\lambda_{1}}{\vec{\lambda}_{2}}=\sqrt{\frac{(a c)}{(a b)}: \frac{(d c)}{(d b)}}
$$

*) Es ist dies eine Verallgemeinerung eines Theorems, welches nach einer Bemerkung des Herrn Lindemann (diese Annalen Bd. VII, S. 66, Anmerk.) für Gerade, die eirem Hyperboloid angehören, bereits von Herrn Stolz ausgesprochen worden ist. 
3) Gleichung des Complexes, dessen Gerade vier feste Ebenen, die ein Tetraeder mit den Kanten $a, b, c, d$ bilden, in constantem Doppelverhältniss $\delta$ schneiden. Wir setzen $a$ und $d$, $b$ und $e$ als Paare gegenüberliegender Kanten voraus. Es bestehen dann die Bedingungen:

$$
\begin{array}{lll}
\left(a^{2}\right)=0, & \left(b^{2}\right)=0, \quad\left(c^{2}\right)=0, & \left(a^{2}\right)=0, \\
(a b)=0, & (a c)=0, \quad(b d)=0, & (a c)=0 .
\end{array}
$$

Eine Gerade $x$ des Complexes möge die vier Seitenflächen des Tetraeders in Punkten schneiden, durch welche die Strahlen

$$
\begin{aligned}
& \text { 1) } a+\lambda c, \\
& \text { 2) } b+\mu d, \\
& \text { 3) } a+v b, \\
& \text { 4) } c+x d
\end{aligned}
$$

gehen. Da

$(a x)+\lambda(b x)=0,(b x)+\mu(d x)=0, \quad(a x)+\nu(b x)=0,(c x)+x(d x)=0$, so besteht zwischen den Parametern die Identität:

$$
\mu \nu=x \lambda \text {. }
$$

Um das Doppelverhältniss der vier Punkte auszudrücken, wäblen wir zwei sich schneidende Strahlen $y, z$, welche $x$ und beziehungsweise 1 ), 2) schneiden, so dass:

$$
\begin{gathered}
(y z)=0, \\
(a y)+\lambda(c y)=0, \\
(b z)+\mu(d z)=0 .
\end{gathered}
$$

Der Strahl $y+\rho_{1} z$ schneide 3$), y+\varrho_{2} z$ dagegen 4), so ist ferner:

und

$$
\begin{aligned}
& (a y)+\nu(b y)+e_{1}[(a z)+\nu(b z)]=0, \\
& (c y)+x(d y)+\varrho_{2}[(c z)+x(d z)]=0
\end{aligned}
$$

$$
\delta=\frac{\rho_{1}}{\rho_{2}}
$$

Nimmt man für $y$ den Strahl $a+\lambda c$, so ist: $(a y)=0,(b y)=0,(c y)=0, \quad(d y)=(a d), \quad(y x)=0,(b z)+\mu(d z)=0$. Vermöge dieser Ausdrücke ergiebt sich sofort:

$$
\frac{e_{1}}{\rho_{2}}=-\frac{\nu}{x} \frac{(b c)}{(a d)}
$$

Man erhält daher etwa aus 3), 4) die Gleichung des Complexes in der Form

$$
a_{x} d_{x}(b c)+\delta(a d) c_{x} b_{x}=0
$$

Insbesondere ist also die Gleichung des harmonischen Complexes

$$
a_{x} d_{x}(b c)-(a d) c_{x} d_{x}=0 \text {. }
$$

Der Complex $a_{x} d_{x}(b c)+(a d) c_{x} d_{x}=0$ ist dagegen ein specieller, alle 
seine Linien sind singulär. Er stellt alle Geraden vor, welche durch die beiden übrigen Kanten des Tetraeders hindurchgehen. Nennen wir dieselben $\gamma^{\prime} \gamma^{\prime \prime}$, so muss sich die letztere Gleichung auf

$$
\gamma_{x}^{\prime} \gamma_{x}^{\prime \prime}=0
$$

reduciren. Man kann dies in der 'That auf folgende Weise zeigen. Die beiden Kanten $\gamma^{\prime} \gamma^{\prime \prime}$ sind bestimmt durch die Gleichungen:

$$
(\gamma a)=0, \quad(\gamma b)=0, \quad(\gamma c)=0, \quad(\gamma d)=0, \quad\left(\gamma^{2}\right)=0 .
$$

Bezeichnet man, wie dies in Folgenden immer geschehen soll, die Determinante

$$
\Sigma \pm\left(A_{1} B_{2} C_{3} D_{4} E_{5} F_{6}\right)
$$

durch $(A B C D E F)$, und wird demgemäss

gesetzt, so ist

$$
\begin{aligned}
& \left(a b c a \gamma^{\prime} \gamma^{\prime \prime}\right)=\Delta, \\
& \left(a b c d x \gamma^{\prime}\right)=\Delta^{\prime}, \\
& \left(a b c d x \gamma^{\prime \prime}\right)=\Delta^{\prime \prime}
\end{aligned}
$$

$$
\begin{aligned}
\Delta^{2} & =\left(\gamma^{\prime} \gamma^{\prime \prime}\right)^{2}(a d)^{2}(b c)^{2}, \\
\Delta^{\prime} \Delta & =\left(\gamma^{\prime} \gamma^{\prime \prime}\right)\left(\gamma^{\prime} x\right)(a d)^{2}(b c)^{2}, \\
\Delta^{\prime \prime} \Delta & =\left(\gamma^{\prime} \gamma^{\prime \prime}\right)\left(\gamma^{\prime \prime} x\right)(a d)^{2}(b c)^{2},
\end{aligned}
$$

also

$$
\begin{aligned}
& \Delta=\left(\gamma^{\prime} \gamma^{\prime \prime}\right)(a d)(b c), \\
& \Delta^{\prime}=\left(y^{\prime} x\right)(a d)(b c), \\
& \Delta^{\prime \prime}=\left(\gamma^{\prime \prime} x\right)(a d)(b c) .
\end{aligned}
$$

Endlich hat man, durch Multiplication der Determinanten $\Delta^{\prime} \Delta^{\prime \prime}$ $\Delta^{\prime} \Delta^{\prime \prime}=-\left(\gamma^{\prime \prime} x\right)\left(\gamma^{\prime} x\right)((a d)(b c))^{2}-2(a d)(b c)\left[a_{x} d_{x}(b c)+(a d) b_{x} c_{x}\right]$ eine Gleichung, welche nach den zuvor bestimmten Werthen von $\Delta^{\prime}$ und $\Delta^{\prime \prime}$ die Identität aussagt*).

$$
a_{x} d_{x}(b c)+(a d) b_{x} c_{x}=-\gamma_{x}^{\prime} \gamma_{x}^{\prime \prime}(a d)(b c)
$$

Ueber die Umformung verschiedener homogener Differentialausdrücke in Liniencoordinaten.

Für unendlich nahe Gerade

$$
x, x+d x, x+2 d x+d^{2} x, x+3 d x+3 d^{2} x+d^{3} x
$$

gelten die folgenden Gleichungen, welche sich durch Differentiation der Identität $\left(x^{2}\right)=0$ ergeben:

๑In ähnlicher Weise lässt sich z. B. auch das Problem behandeln, denjenigen Comp ex au bestimmen, dessen Gerade von sechs festen Ebenen in einer Involution geschnitten werden. 


$$
\begin{aligned}
\left(x^{2}\right) & =0 \\
(x d x) & =0 \\
\left(x d^{2} x\right)+\left(d x^{2}\right) & =0 \\
\left(x d^{3} x\right)+3\left(d x d^{2} x\right) & =0 \text { u. s. w. }
\end{aligned}
$$

Die Gleichung $(x d x)=0$ sagt daber nicht aus, dass zwei unendlich nahe Gerade sich schneiden. Dies tritt in der That erst dann ein, wie Herr Klein bereits gezeigt hat ${ }^{*}$, wenn auch die Gleichung

erfüllt ist.

$$
-\left(x d^{2} x\right)=\left(d x^{2}\right)=0
$$

Wir betrachten nun die Geraden, welche drei Complexen $F \Phi \psi$ bez. von den Graden $m n p$ angehören. Dabei setzen wir wie üblich

$$
\begin{aligned}
& f_{i}=\frac{1}{m} \frac{\partial F}{\partial x_{i}}, \\
& f_{i k}=\frac{1}{m m-1} \frac{\partial^{2} F}{\partial x_{i} \partial x_{k}},
\end{aligned}
$$

so dass

$$
\begin{aligned}
& \Sigma x_{i} f_{i}=F, \\
& \Sigma x_{i} f_{2 k}=f_{k} .
\end{aligned}
$$

Dabei werden wir im Folgenden das Summenzeichen fortlassen, also statt der angeführten Ausdrücke einfach schreiben $x_{v} f_{i}=F, x_{i} f_{i k}=f_{k}$ u. s. w. Ferner sollen Ausdrücke, wie $\Sigma\left(f_{i}\right)^{2}, \Sigma f_{i} \varphi_{i}, \Sigma f_{i} \psi_{i}$ und ana$\log$ gebildete durch $f^{2}, f \varphi, f \psi$ bezeichnet werden.

Für eine den drei Complexen angehörige Gerade $x$ ist nun

$$
F=0, \quad \phi=0, \quad \psi=0 \text {. }
$$

Für eine unendlich nahe (consecutive) gelten die Gleichungen:

$$
\left\{\begin{array}{l}
(f d x)=0, \\
(\varphi d x)=0, \\
(\psi d x)=0, \\
(x d x)=0, \\
(k d x)=0 .
\end{array}\right.
$$

In ihnen bedeaten die $k_{i}$ beliebige Coefficienten, welche die homogenen Differentiale verbinden. Die Gleichungen (2) bestimmen die Verhältnisse der $d x_{i}$ als Unterdeterminanten, so dass, wenn

gesetzt wird

$$
\Pi=(f \varphi \psi x k \alpha)
$$

$$
d x_{1}: d x_{2}: d x_{3}: d x_{4}: d x_{5}: d x_{6}=\frac{\partial \pi}{\partial \alpha_{1}}: \frac{\partial \pi}{\partial \alpha_{2}}: \frac{\partial \pi}{\partial \alpha_{3}}: \frac{\partial \pi}{\partial \alpha_{4}}: \frac{\partial \pi}{\partial \alpha_{3}}: \frac{\partial \pi}{\partial \alpha_{8}} .
$$

Sie geben zu verschiedenen Formeln Veranlassung, von welchen

*) Klein, Math. Ann. Bd. V, S. 293. 
in der Liniengeometrie ein sehr vielfältiger Gebrauch zu machen ist und deren wichtigste wir im Folgenden darlegen werden.

Wir setzen:

$$
(d x f \varphi \psi x k)=\left(d x^{2}\right)=\Delta \text {. }
$$

Quadrirt man $\Delta$, so ergiebt sich mit Rücksicht auf die Gleichungen $(2),(1) \cdot$

wo zur Abkürzung

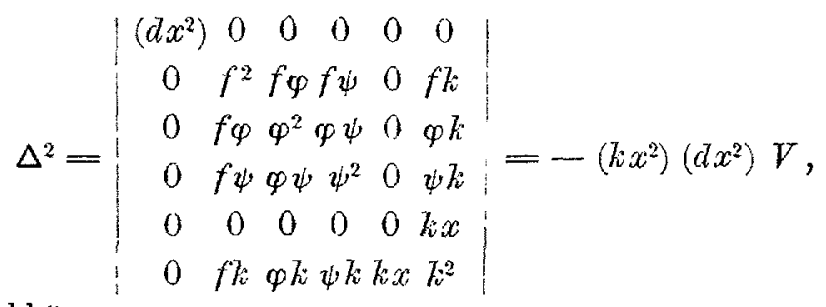

gesetzt ist. Es ist daher

$$
V=\left|\begin{array}{ccc}
f^{3} & f \varphi & f \psi \\
f \varphi & \varphi^{2} & \varphi \psi \\
f \psi & \varphi \psi & \psi^{2}
\end{array}\right|
$$

(5)

$$
\Delta=-\left(k x^{2}\right) V \text {. }
$$

Setat man ferner

so ergiebt sich sofort

$$
\Delta^{\prime}=\left(f \varphi \psi x d x d x^{2}\right)
$$

also

$$
\Delta \Delta^{\prime}=\left(d x^{2}\right)\left(d x^{2}\right)(k x) V
$$

$$
\Delta^{\prime}=-\left(k x^{3}\right) \nabla^{2}
$$

Bedeuten die $c_{i} b_{i}$ willkührliche Grössen, und wird die Determinante gesetzt, so ist

$$
(f \varphi \psi x c b)=\mathrm{K}
$$

so dass

$$
\mathrm{k} \Delta^{\prime}=-\left(a x^{2}\right) V: \begin{array}{lll}
c x & e_{d x} \\
b x & b_{a x}
\end{array} \mid
$$

$$
\left|\begin{array}{lll}
c x & c_{a x} \\
b x & b_{d x}
\end{array}\right|=-(k x)(f \varphi \psi x \in b)=-(k x) \mathrm{K} .
$$

Endlich hat man als Werth der Determinante, welcher entsteht, wenn man die Determinante der quadratischen Form $\left(x^{2}\right)$ mit $f_{i} \varphi_{i} \psi_{i} x_{i} e_{i}$ horizontal und mit $f_{i} \varphi_{\imath} \psi_{i} x_{i} b_{i}$ vertical rändert, eine Operation, die wir durch

bezeichnen,

$$
\left(\Omega: f_{i} \varphi_{i} \psi_{i} x_{i} \begin{array}{c}
e_{i} \\
b_{i}
\end{array}\right)
$$

$$
\left(\Omega: f_{i} \varphi_{i} \dot{\psi}_{i} x_{i}^{b_{i}} b_{i}\right)=-c_{z} b_{x} V \text {. }
$$

Die consecutive Gerade von $x+d x, x+2 d x+d^{2} x$ ist bestimmt durch die Gleichungen: 


$$
\left\{\begin{array}{l}
k_{i} d^{2} x_{i}=0 \\
x_{i} d^{2} x_{i}+\left(d x_{i}^{2}\right)=0 \\
f_{i} d^{2} x_{i}+(m-1) f_{i k} d x_{i} d x_{k}=0 \\
\varphi_{i} d^{2} x_{i}+(n-1) \varphi_{i k} d x_{i} d x_{k}=0 \\
\psi_{i} d^{2} x_{i}+(p-1) \psi_{i k} d x_{i} d x_{k}=0 .
\end{array}\right.
$$

Trägt man an Stelle der $d x_{i} d x_{k}$ ihre Werthe aus (2) ein, so entsteht:

$$
f_{i} d^{2} x_{i}=-(n-1)\left|\begin{array}{lllllllllll}
f_{11} & f_{12} & f_{13} & f_{14} & f_{15} & f_{16} & f_{1} & \varphi_{1} & \psi_{1} & x_{1} & k_{1} \\
f_{21} & f_{22} & f_{23} & f_{24} & f_{25} & f_{26} & f_{2} & \varphi_{2} & \psi_{2} & x_{2} & k_{2} \\
f_{31} & f_{32} & f_{33} & f_{34} & f_{35} & f_{36} & f_{3} & \varphi_{3} & \psi_{3} & x_{3} & k_{3} \\
f_{51} & f_{42} & f_{43} & f_{41} & f_{45} & f_{46} & f_{1} & \varphi_{4} & \psi_{4} & x_{4} & k_{4} \\
f_{51} & f_{52} & f_{53} & f_{54} & f_{55} & f_{56} & f_{5} & \varphi_{5} & \psi_{5} & x_{5} & k_{5} \\
f_{61} & f_{62} & f_{63} & f_{64} & f_{65} & f_{66} & f_{6} & \varphi_{6} & \psi_{6} & x_{6} & k_{6} \\
f_{1} & f_{2} & f_{3} & f_{4} & f_{5} & f_{6} & 0 & 0 & 0 & 0 & 0 \\
\varphi_{1} & \varphi_{2} & \varphi_{3} & \varphi_{4} & \varphi_{5} & \varphi_{6} & 0 & 0 & 0 & 0 & 0 \\
\psi_{1} & \psi_{2} & \psi_{3} & \psi_{4} & \psi_{5} & \psi_{6} & 0 & 0 & 0 & 0 & 0 \\
x_{1} & x_{2} & x_{3} & x_{4} & x_{5} & x_{6} & 0 & 0 & 0 & 0 & 0 \\
k_{1} & k_{2} & k_{3} & k_{4} & k_{5} & k_{6} & 0 & 0 & 0 & 0 & 0
\end{array}\right|
$$

Man reducirt diese Determinante, indem man die sechs exsten Horizontal- oder Vertikalreihen mit den $x$ multiplicirt and von der siebenten abzieht. Es ergiebt sich so:

$$
\left\{\begin{array}{l}
f_{i} d^{2} x_{i}=(m-1) U_{1}(k x)^{2}, \\
\varphi_{i} d^{2} x_{i}=(n-1) U_{2}(k x)^{2}, \\
\psi_{i} d^{2} x_{i}=(p-1) U_{3}(k x)^{2} .
\end{array}\right.
$$

Es bedeutet in den Gleichungen (10) $U_{1}$ - und dem analog $U_{2}, U_{3}$ - die mit $\varphi_{i} \psi_{i} x_{i}$ geränderte Determinante der Coefficienten der quadratischen Form

$$
\boldsymbol{X}_{i} \boldsymbol{X}_{k} f_{i k}
$$

welche letztere mit der Hesse'schen Determinante von $F$ identisch ist.

Die Darstellung des Ausdruckes $d x_{i} d^{2} x_{i}=-\frac{1}{3}\left(x_{i} d^{3} x_{i}\right)$ erhält man durch Differentiation der Gleichung (5), nämlich:

$$
-2\left(d x d^{2} x\right)=(k x)^{2}\left(\frac{\partial V}{\partial x_{i}} d x_{i}\right)
$$

Andererseits hat man:

$$
d x d^{2} x=\left(d^{2} x f \varphi \ddot{\psi} x k\right)=\Delta^{\prime \prime \prime}
$$

Darans ergiebt sich: 


$\left(\Delta^{\prime \prime \prime}\right)^{2}=\left(\begin{array}{cccccc}\left(d^{2} x^{2}\right) & (m-1)(k x)^{2} U_{1}(n-1)(k x)^{2} U_{2}(p-1)(k x)^{2} U_{3} & (k x)^{2} V & 0 \\ (m-1)(k x)^{2} U_{1} & f^{2} & f \varphi & f \psi & 0 & f k \\ (n-1)(k x)^{2} U_{2} & f \varphi & \varphi^{2} & \varphi \psi & 0 & \varphi k \\ (p-1)(k x)^{2} U_{3} & f \psi & \varphi \psi & \psi^{2} & 0 & \psi k \\ (k x)^{2} V & 0 & 0 & 0 & 0 & (k x \\ 0 & f k & \varphi k & \psi k & (k x)^{2} & h^{2}\end{array}\right.$

Aus diesem Ausdrucke lassen sich die willkührlichen Grössen $k$ ohne weiteres nicht entfernen. Wenn aber $V=0$, so erhält man:

$$
\left.\left(\Delta^{\prime \prime \prime}\right)^{-}=(k x)^{6}\right)\left|\begin{array}{cccc}
0 & (m-1) U_{1} & \left(n-1 U_{2}\right) & (p-1) U_{3} \\
(m-1) U_{1} & f^{2} & f \varphi & f \psi \\
(n-1) U_{2} & i f \varphi & \varphi^{2} & \varphi \psi \\
(p-1) U_{3} & f \psi & \varphi \psi & \psi^{2}
\end{array}\right|
$$

Der Ausdruch rechter Hand lässt sich, von der Gleichung (11) ausgehend, wenn man die Differentialquotienten von $V$ mit $v_{i}$ bezeichnet, auch in folgender Form

darstellen.

$$
\frac{1}{\frac{1}{4}(k x)^{b}}: \begin{array}{cccc}
0 f & f^{2} & f \varphi & f \psi \\
v \varphi & f \psi & \varphi^{2} & \varphi \psi \\
v \psi & f \psi & \varphi \psi & \psi^{2}
\end{array} \mid
$$

III.

Die Ordnung und der Rang der windschiefen Fläche, welche vollständiger Schnitt der drei Complexe $F \Phi \psi$ ist.

Die Ordnung $N$ der windschiefen Fläche ergiebt sich gleich $2 \mathrm{mnp}$. Eine willkürliche Gerade $y$ wird närulich von so viel Frrzeugenden $x$ der Fläche getroffen als das System der Gleichungen

$$
(y x)=0, \quad F=0, \Phi=0, \quad \Psi=0, \quad\left(x^{2}\right)=0
$$

lösungen für $x$ zulässt.

Die Gleichungen II. (2) zeigen ferner, dass die consecutive Erzeugende einer beliebigen $x$ vollkommen bestimmt ist. Eine Unbestimmtheit tritt nur dann ein, wenn Gleichungen von der Form

$$
\mu_{1} f_{i}+\mu_{2} \varphi_{i}+\mu_{3} \psi_{i}=x_{i} \quad i=1,2,3,4,5,6
$$

bestehen. Die consecutive Erzeugende ist dann aus den Gleichungen: 


$$
\left\{\begin{array}{c}
(x d x)=0 \\
(f d x)=0 \\
(\varphi d x)=0 \\
(\psi d x)=0 \\
(k d x)=0 \\
\left(\mu_{1}(m-1) f_{i k}+\mu_{2}(n-1) \varphi_{i k}+\mu_{3}(p-1) \psi_{i k}\right) d x_{i} d x_{k}=T_{i k} d x_{i} d x_{k}
\end{array}\right.
$$

zu bestimmen. Darnach ergeben sich zwei Werthsysteme für die Differentiale. Die Fläche hat also eine Doppelerzeugende, wenn die Gleichungen (1) exfüllt sind. Fügt man den Gleichungen (2) noch die folgenden

$$
(z x)=0, \quad(z d x)=0
$$

hinzu und eliminirt die Differentiale, so erhält man das Product der beiden -Gleichungen zweier linearer Complexe, denen die Doppelerzeugende angehört, und deren Ebenen längs derselben Tangentenebenen der Fläche sind. Dieses Product lässt sich, wenn durch $\Pi$ die Determinante der quadratischen Form $T_{i k} x_{i} x_{k}$ bezeichnet wird, darstellen durch

$$
\left(\pi: f_{i} \varphi_{i} z_{i}\right)=0
$$

Die Doppelerzeugende wird daher zur Rüekkehrerzeugenden, wenn der Kern dieser Determinante versehwindet, d. h. wenn

$$
\left(\Pi: f_{i} \varphi_{i}\right)=0 \text {. }
$$

Höhere Singu[aritäten der Fläche entstehen, wemn von den Gleichungen

$$
f_{i}=\varrho_{1} x_{i}, \quad \varphi_{i}=\varrho_{2} x_{i}, \quad \psi_{i}=\varrho_{3} x_{i}
$$

zwei oder drei für eine Erzeugende $x$ bestehen. Besteht nur die Gleichung $f_{i}=\varrho_{1} x_{i}$, so hat der erzeugende Complex $F$ selbst $x$ zur Doppellinie*), die dann Doppel- oder Rückkehrerzeugende der Fläehe wird. Finden dagegen awei oder drei zagleich statt, so hat die Fläche die Gerade $x$ zur vier-, respective achtfachen Generatrix.

Die Gleichungen (1) repräsentiren eine Bedingungsgleichung. zwischen den Coefficienten der drei Complexe. Für den Fall

$$
\begin{aligned}
& F=\Sigma a_{i k} x_{i} x_{k}, \\
& \Phi=\gamma^{\prime} x, \\
& \psi=\gamma^{\prime \prime} x
\end{aligned}
$$

kann man dieselbe dahin aussprechen, dass die Discriminante nach $\mu$ der Determinante:

*) Plücker, Neue Geometrie des Ráumes S. 296. 
Zur Theorie der windschiefen Flächen.

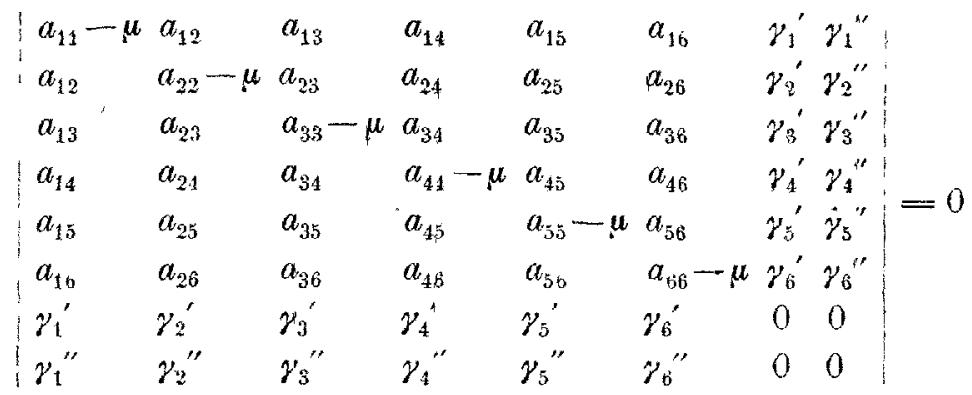

verschwinden muss.

Unter dem Range einer Fläche verstehen wir überbaupt den Grad desjenigen specieller Complexes, welcher die sämmtlichen Tangenten derselben repräsentirt, oder den Grad der Gleichung der Fläche in Liniencoordinaten. Der Rang ist daher auch gleich dem Grade ihres Tangentenkegels oder gleich der Classe ihrer ebenen Schnittcurve.

Die Gleichung der windschiefen Fläche erhält man auf folgende Weise ausgedrückt in Liniencoordinaten. Soll die Gerade $y$ Tangente der Fläche sein, so muss sie zwel consecutive Erzeugende derselben schneiden. Daher ist

$$
\left\{\begin{array}{l}
(y x)=0, \\
y d x)=0 .
\end{array}\right.
$$

Eliminirt man aus den Gleichungen II. (2) und der letzteren die Differentiale, so erhält man

$$
(f \varphi \psi x k y)=0 \text {. }
$$

Das Quadrat des letzteren Ausdruckes ist gleich $(k x)^{2}$, multiplicirt in die mit $f y \varphi y \psi y$ geränderte Determinante $V$. Es lässt sich also aus (4) der Factor $(k x)$ stets durch Division aussondern, wenngleich dies, wie es scheint, nicht so ausgeführt werden kann, dass die Determinantenform dabei beibehalten wird. Die Gleichung: (4) ist daher vom ersten Grade in $y$, vom $m+n+p-3^{\text {ten }}$ in $x$. Eliminirt man $x$ aus (4) und den Gleichungen

$$
(y x)=0, \quad\left(x^{2}\right)=0, \quad F=0, \quad \Phi=0, \quad \psi=0,
$$

so entsteht eine Gleichung vom Grade

$$
R=2 m n p(m+n+p-2)
$$

in den $y$, welche die Fläche in Liniencoordinaten $y$ darstellt. Für jede Doppelerzeugende $x_{0}$ der Fläche enthält dieselbe quadratisch den Factor $\left(x_{0} y\right)$.

Wir zeigen, wie eine solche Elimination in den einfacheren Fällen sich wirklich ausführen lässt. 
1) Linienfläche $(1,1,1)$ (nach Plüeker's Bezeichnung*), velche durch arei lineare Complexe $a_{x}=0, b_{x}=0, c_{x}=0$ erzengt wird.

Man hat hier ohne weiteres:

$$
(a b c x k y)^{2}=(k x)^{2}\left|\begin{array}{llll}
a^{2} & a b & a c & a y \\
a b & b^{2} & b c & b y \\
a c & b c & c^{2} & c y \\
a y & b y & c y & 0
\end{array}\right|=0 .
$$

Das Quadrat des Ausdruekes (4) von dem Factor (kx) befreit, stellt also einen von den $x$ völlig freien Ausdruck, d. h. das Eliminationsresultat oder die Gleichung des ron den drei linearen Complexen erzengten Hyperboloides in Liniencoordinaten vor.

2) Linienfläche $(2,1,1)$, welche durch den Schnitt areier Complexe $a_{x}=0, b_{x}=0 ; \psi=\Sigma a_{i k} x_{i} x_{k}=0$ erzengt wird. Die Gleichuag:

$$
\left|\begin{array}{cccc}
a^{2} & a b & a \psi & a y \\
a b & b^{2} & b \psi & b y \\
a \psi & b \psi & \psi^{2} & \psi y \\
a y & b y & \psi y & 0
\end{array}\right|=0
$$

ist homogen rom zweiten Grabe in $y$ und $x$. Multiplieirt man die Gleichungen $(y x)=0,(a x)=0,(b x)=0$ mit $x_{1} x_{2} x_{3} x_{4} x_{5} x_{6}$ und nimmt $\left(x^{2}\right)=0, \psi=0$ hinzu, so hat man 21 Gleichungen, in welchen die 21 unbekannten Grössen $x_{i} x_{\bar{k}}$ linear und homogen auftreten. Die Determinante dieser 21 Gleichungen, gleich Null gesetat, stellt die Gleichung der Fläche in Liniencoordinaten ror, da sie die $y$ nur im achten Grade enthäl. Man vermeidet das Auftreten einer so vielreihigen Determinante, wenn man zuvor die Gleichung (4) duxch Division von dem Factor $(k x)$ befreit. Man hat alsdann aus zwei Gleichungen zweiten und vier Gleichungen ersten Grades die $x$ zu eliminiren, was ohne Schwierigkeit geschehen kann**), weshalb wir nicht weiter darauf eingehen.

Wir wenden uns jetat wieder zu der allgemeinen Betrachtung zuräck. Bezeichnet man wit $d$ die Zabl der Doppelerzeugenden der Fläche, so ist wach (5) der Rang der Fläche gegeben durch die Gleichung

$$
R=2 m n p(m+n+p-2)-2 d \text {. }
$$

Man kann die Zahl $R$ auch durch directe Bestimmung der Classe der ebenen Schnittcurve oder der Ordnung des Tangentenkegels er-

*) Plü cker, Sur les surfaces reglées leur construction et classification, Ann. di Mat 1870.

**) VgL Clebsch, Crelle's Journ. Bd. 59, S. 1, oder auch. Ueber eine Classe von Eliminationsproblemen Bd. 58 desselben Journals. 
halten. Soll eine Gerade $a+\lambda b$ des ebenen Büschels $(a, b)$ Tangente der Fläche sein, so ist nach (3):

daher

$$
\begin{aligned}
a_{x}+\lambda b_{x} & =0, \\
\left(a_{d x}\right)+\lambda\left(b_{d x}\right) & =0,
\end{aligned}
$$

$$
a_{x}\left(b_{d x}\right)-b_{x}\left(a_{d x}\right)=0 \text {. }
$$

Werden jetzt die Differentiale eliminirt, so ist

$$
\left(f \varphi \psi x k_{i} \quad a_{x} b-b_{x} a\right)=0
$$

eine Gleichung, welche einen Complex $m+n+p-2^{\text {ten }}$ Grades vorstellt, welcher die erzeugenden Complexe in $2 m n p(m+n+p-2)$ Geraden schneidet. Derselbe geht ersichtlich durch jede Doppelerzeugende der Fläche einmal hindurch, da der Ausdruck (6) verschwindet, wenn die Gleichungen (1) gelten. Die Classe des ebenen Schnittes, sowie der Grad des Tangentenkegels ist daher durch die Zahl $R$ gegeben $*$ ).

Bezeichnet man den Grad der Doppelaurve der Fläehe durch $D$, so ist nach den Formeln von Plücker

$$
N(N-1)-2 D-2 d=R,
$$

also, wenn für $N 2 m n p$, für $R$ die Zahl $2 m n p(m+n+p-2)-2 d$ gesetzt wird:

$$
D=m n p[2 m n p-(m+n+p)+1] .
$$

Man erhält hieraus endlich das Geschlecht $P$ der windschiefen Fläche

$$
\begin{aligned}
P & =\frac{1}{2}(N-1)(N-2)-D-a \\
& =(2 m n p-1)(m n p-1)-D-d \\
& =m n p(m+n+p-4)+1-d .
\end{aligned}
$$

Das Auftreten von Doppelerzengenden modificirt daher den Grad der Doppelcurve nicht, sondern nur das Geschlecht der Fläche. Die Linienfiächen $(2,1,1)$ sind im Allgemeinen vierten Grades rom Geschlechte eins, mit einer Doppelcurve zweiten Grades, welche erhalten bleibt, wenn die Fläche eine Doppelerzeugende erhält, also das Geschlecht Null wird.

Wir verificiren die formeln $(7),(8)$ in einigen Fällen durch directe Betrachtung.

1) Linienfläche $(p, 1,1)$. Zwei lineare Complexe $a_{x}=0, b_{x}=0$ und $\psi=0$ erzeugen eine windschiefe Fläche $(p, 1,1)$ vom Grade $2 p$, deren Rang $2 p^{2}$ ist. Man kann die Classe des ebenen Schnittes aber auch auf folgende Weise finden. Die Fläche enthält nämlich zwei

*) Auf eine hiervon nicht wesentlich verschiedene Weise hat bereits Herr Lüroth die Zahl $R$ bestimmt. Crelle's Journ. Bd. 67, S. 135 . 
$p$-fache Leitlinien, die Directricen des den beiden Complexen $a_{x}, b_{x}$ zugehörigen Büschels and ausser ihnen offenbar keine Doppelcurve. Daher ist der ebene Schnitt eine Curve $2 p^{\text {ter }}$ Ordnung mit zwei $p$-fachen Punkten, seine Classe also wie vorhin:

$$
2 p(2 p-1)-2 p(p-1)=2 p^{2} \text {. }
$$

2) Linienfläche $(p, 2,1)$, welche von einem speciellen linearen, einem Complexe zweiten Grades, dem die Axe a des speciellen angehört, und $\psi=0$ erzeugt wird. Die Singularitäten der Fläche sind folgende: Die Axe $a$ ist $2 p$-fache Leitlinie derselben. Durch dieselbe gehen ferner nach den Untersuchungen Plücker's *) vier ansgezeichnete Ebenen $e_{1} e_{2} e_{3} e_{4}$, für welche die in ihnen liegende Complexcurve des Complexes zweiter Ordnung in zwei Strablbüschel degenerirt, deren Centra beziehungsweise $a_{1} \omega_{1} a_{2} \omega_{2} o_{3} \omega_{3} \sigma_{4} \omega_{4}$ seien. Von denselben liegen $O_{1} O_{2} O_{3} O_{4}$ auf der Axe a selbst. Die Doppelcurve hat daher diese Punkte zu vielfachen Punkten, welche, wie man leicht sieht, als $\frac{p(p-1)}{2}$ fache zu zählen sind, weil ron ihnen so viel Zweige der Curve anslaufen. In einer beliebig durch die Axe gelegten Ebene liegen ferner $2 p$ Erzeugende der Kläche - die gemeinsamen Tangenten des Complexkegelschnittes and der Complexcurve von $\psi=0$ - also $(2 p-1) p$ Punkte der Doppelcurve. Daher ist der Grad der letzteren

$$
2 p(p-1)+p(2 p-1)=4 p^{2}-3 p
$$

der Totalgrad der Doppelcurven ist also, wenn man die $2 p$-fache Leitlinie noch hinzurechnet:

$$
6 p^{2}-4 p
$$

eine Zahl, welche sich übereinstimmend auch aus (7) ergiebt. Die Doppeleurve hat übrigens auch die vier Punkte $\omega_{1} \omega_{2} \omega_{3} \omega_{4}$ za $\frac{p(p-1)}{2}-$ fachen Punkten, während die $\frac{p(p-1)}{2} Z$ weige derselben, welche von einem der Punkte $o_{i}$ auslaufen, von der zugehörigen Ebene $e_{i}$ in demselben berührt werden. Beispielsweise ist für $p=3$ die krumme Doppeleurve der Fläche zwölfter Ordnung von der $27^{\text {ten }}$ Ordnung mit 8 wirklichen dreifachen Punkten.

Die Formel (7) nimmt eine etwas andere Gestalt an, wenn die Doppelerzeugende zugleich einen Theil der Doppelcurve der Fläche ausmacht. Um diesen Fall zu erläutern, betrachten wir die windschiefe Fläche, welche ron einem speciellen linearen Complexe $\gamma_{x}$, dessen Axe $\gamma$ den beiden Complexen $\Phi, \Psi$ angehört, gebildet wird. Die Gleichangen III. (2), welche die consecutive Erzeugende $x+d x$ bestimmen, werden unbestimmt für $x=\gamma$; sie sind dann zu ersetzen durch das System:

*) Plücker, N. Geom. d. R, S. 177. 


$$
\begin{aligned}
& (\gamma d x)=0, \\
& (\varphi d x)=0, \\
& (\psi d x)=0, \\
& (h d x)=0, \\
& \left(d x^{2}\right)=0 .
\end{aligned}
$$

Aus denselben ergiebt sich, dass $\gamma$ Doppelerzengende der Gílächa ist und zugleich von ihren beiden consecutiven Erzeugenden geschnitten wird. Ausserdem ist aber $\hat{\gamma}$ eine $n p-1$-fache Leitgerade der Fläche. Der Rang derselben ist nach wie vor durch die Gleichung (6)

$$
P=\left(\left(a_{x} b-b_{x} a\right) \varphi \psi \gamma x k\right)=0
$$

bestimmt. $P$ verschwindet für $x=\gamma$, während $\frac{\partial P}{\partial x_{i}}$ einen vestimmten Werth behält. Da auch $\Sigma \frac{\partial P}{\partial x_{i}} d x_{i}$ nicht verschwindet, findet für de Rang der Fläche keine weitere Erniedrigung statt; er ist demnach:

$$
2 n p(n+p-1)-2 \text {. }
$$
- chung

Der Grad der krummen Doppelcarve $D$ ist daher durch die Glei-

$$
D=\frac{n p}{2}(3 n p-1)-n p(n+p)+1
$$

gegeben. Das Geschlecht der Fläche bestimmt sich noch immer nach (8)

$$
P=n p(n+p-3) \text {. }
$$

IV.

Die singulären Erzeugenden der windschiefen Fläche.

Zwei consecutive Erzeugende der Fläche schneiden sich im Allgemeinen nicht. Damit $x$ von $x+d x$ getroffen werde, muss nach II. die Bedingung

$$
\left(d x^{2}\right)=-(k x)^{2} \nabla=0
$$

erfüllt sein. $\quad V=0$ stellt einen Complex vom Grade $2(m+n+p-3)$ vor, welcher mit den erzeugenden Complexen $4 m n p(m+n+p-3)$ singuläre Erzeugende der Fläche bestimmt*). Der Durchschnittspunkt von $x$ and $x+d x$ liegt dabei auf der Doppeleurve; wir bezeichnen ihn wie üblich als Cuspidalpunkt der Fläche**).

*) Vgl. Lüroth a. a. O.; Klein, Math. Ann. Bd. V, S. 292.

*) Aus der Zahl $4 m n p(m+n+p-3)$ der Cospidalpunkte erhält man leicht die Bestimmung mehrerer anderer, für die Theorie der Liniengebilde wichtiger Zahlen

1) Ordnung und Classe der Brennfäche von zwei Complexer $\Phi=0, \psi=0$. Eine solche Fläche ist in sich dualistisch; sie entsteht, als Punktgebilde betrachtet. 
Befinden sich unter den Complexen zwei lineare $a_{x}, b_{x}$, so gehört die Linienfläche einem ganzen Büschel linearer Complexe $a_{x}+\lambda b_{x}=0$ an. In dem Letzteren befinden sich im Allgemeinen zwei specielle; die Fläche hat die Axen $\alpha, \beta$ derselben zu $p$-fachen Leitlinien, auf denen je $2 p(p-1)$ Cuspidalpankte liegen.

Inshesondere aber können die beiden Axen $\alpha, \beta$ zusammenfallen. Die Fläche gehört dann einer speciellen Congruenz an, man kann sie erzengen durch den Complex $\psi$, den speciellen linearen $\alpha_{x}$ und einen anderen linearen, der die Axe $\alpha$ in sich enthält. Sie hat dann zwei

durch die Schnitte unendlich naher Geraden der Congruenz $\Phi=0, \Psi=0$, als Ebenengebilde durch die Ebenen dieser Geraden, wobei aber im Allgemeinen die betrefende Ebere ihren Tangentialpunkt nicht im Sebnittpunkte der beiden Geraden hat. Vgl. Pasch, Habilitationsschrift, Giessen 1870.

Die Zahl der singulären Erzeugenden der Linienfläche, welche von $\Phi, \psi$ und einem speciellen linearen Complexe gebildet wird, ist nun gleich der Summe der Zabl der Punkte der Brennfläche, welche auf' der Axe des letzteren liegen, und der Ebenen der Brennfläche, welche durch die Axe gehen, $d$. b. gleich der Summe von Ordnung und Classe der Brennfïche. Da beide Zahlen gleich sein müssen, so hat man

$$
2 n p(n+p-2)
$$

als Ordmungs- und Classenzahl der Brenufläche zweier Complexe nten und pien Grades (eines Strahlensystems [np]).

2) Ordnung wnd Clewse der singulären Fläche eines Complexes $F=0$. Einem Complexe $F=0$ rom $n^{\text {ten }}$ Grade ist zugeordnet der Complex $\Phi=\Sigma f_{i}^{2}=0$ vorn $2(n-1)^{\text {ters }}$ Grade, welcher mit ihm die Congruenz der singulären Linien des Complexes betimmt. Damit wird zugleich jeder singulären linie $x$ eine andere Linie mit den Coordinaten $y_{i}=f_{i}$ zugeordnet. Beide schneiden sich im singulären Punkte von $x$, welcher ein Punkt der singulären Fläche des Complexes ist, während (xy) die Tangentíalebene dieses Punktes, die singuläre Ebene rorstellt. Für die LinienAäche, welche von $F, \Phi$ and aem linearen speciellen $\Psi=0$ mit der Axe $\alpha$ erzengt wird, ist nun:

$$
V=(f a)\left[2(f \varphi)(\varphi a)-\varphi^{2} \cdot(f a)\right] .
$$

Der Factor ( $f a$ ) gleich Null gesetzt, bestiumt mit den gegebenen Complexen $4 \cdot n(n-1)^{3}$ singuläre Erzeugende der Linienfiäche. Fùr dieselben schneiden sawabl $x$ als $y$ die Axe $a$. Es liegt also entøeder der singuläre Punkt von $x$ aut der Axe, oder die singnäre Ebene $x y$ geht darch dieselbe. $4 n(n-1)^{2}$ ist daher die Summe von Ordnung and Classe der singulären Fläche des Complexes. Mar hat daher den Satz:

Ordnung und Classe der singularen Fläche eines Complecces vam wten Grade ist

$$
2 n(n-1)^{2} \text {. }
$$

Die wämliche Zahl ist anf ganz anderem Wege von $\mathrm{Clebsch}$ gefunden worden. (Vgl. dessen Aufsatz (diese Annalen Bd. V) Ueber die Plücker'schen Complexe und die Singularitätenfiachen derselben.)

Dex andere Factor von $V$ bezieht sich auf Ordnung und Classe einer aweiten Fläche, welche mit der besprochenen die vollständige Brenafläche der Congruenz $F \Phi$ ausmacht.

Oramung und Classe aieser Filäche ist daher

$$
2 n(n-1)\{5 n-7) \text {. }
$$


unendlich nahe $p$-fache Leitlinien. Dem entsprechend geht die Determinante $V$ in ein Quadrat über; es giebt also nur $2 p(p-1)$ - in gewissem Sinne doppeltzählende - singuläre Linien oder Cuspidalpunkte. Diese Bemerkung ist übrigens nur ein specieller fall eines allgemeineren Theorems, welches Herr Klein*) bereits erwähnt hat.

Die Zahl der singulären Ebenen verringert sich, wenn die Fläche eine der in $\$$ III. betrachteten Singularitäten besitzt.

Für eine Doppelerzengende findet, wie wir sahen, die Beziehung:

$$
\mu_{1} f_{i}+\mu_{2} \varphi_{i}+\mu_{3} \psi_{i}=x_{i}
$$

statt. Multiplicirt man die sechs Gleichungen (1) der Reihe nach mit $f_{i} \varphi_{i} \psi_{\imath}$ und summirt jedesmal nach $i$, so ergeben sich die Gleichungen:

$$
\left\{\begin{array}{l}
\mu_{1} f^{2}+\mu_{2} f \varphi+\mu_{3} f \psi=0 \\
\mu_{1} f \varphi+\mu_{2} \varphi^{2}+\mu_{3} \varphi \psi=0 \\
\mu_{1} f \psi+\mu_{2} \varphi \psi+\mu_{3} \psi^{2}=0
\end{array}\right.
$$

Die Determinante derselben ist $V=0$. Der Complex $V$ verschwindet daher für jede Doppelerzeugende der Fläche. Eine weitere Singularität besitzt derselbe im Allgemeinen nicht. Multiplicirt man nämlich die Gleichungen (1) mit $f_{i k}, \varphi_{i k}, \psi_{i k}$ und summirt wieder nach $i$, so ergiebt sich:

$$
\left\{\begin{array}{l}
\mu_{1} f_{i} f_{i k}+\mu_{2} \varphi_{i} f_{i k}+\dot{\mu}_{3} \psi_{2} f_{i k}=f_{k}, \\
\mu_{1} f_{i} \varphi_{i k}+\mu_{2} \varphi_{i} \varphi_{i k}+\mu_{3} \psi_{i} \varphi_{i k}=\varphi_{k} \\
\mu_{1} f_{i} \psi_{i k}+\mu_{2} \varphi_{i} \psi_{i k}+\mu_{3} \psi_{i} \psi_{i k}=\psi_{k}
\end{array}\right.
$$

Werden jetzt die Gleichungen (3) der Reihe nach mit

$$
2 \mu_{1}(m-1), 2 \mu_{2}(n-1), 2 \mu_{3}(p-1)
$$

multiplicirt and addirt, so entsteht:

$$
\text { (4) } \begin{aligned}
R & =\mu_{1}^{2} \frac{\partial f^{2}}{\partial x_{k}}+\mu_{2}{ }^{2} \frac{\partial \varphi^{2}}{\partial x_{k}}+\mu_{3} 2 \frac{\partial \psi^{2}}{\partial x_{k}}+2 \mu_{1} \mu_{2} \frac{\partial f \varphi}{\partial x_{k}}+2 \mu_{1} \mu_{3} \frac{\partial f \psi}{\partial x_{k}}+2 \mu_{2} \mu_{3} \frac{\partial \varphi \psi}{\partial x_{k}} \\
& =2\left[\mu_{1}(m-1) f_{k}+\mu_{2}(n-1) \varphi_{k}+\mu_{3}(p-1) \psi_{k}\right] .
\end{aligned}
$$

Andererseits zeigt die Differentiation von $V$, dass

$$
\frac{\partial V}{\partial x_{k}}=R\left|\begin{array}{ll}
f^{2} & f \varphi \\
f \varphi & \varphi^{2}
\end{array}\right|
$$

Der Complex $V$ hat die Doppelerzeugende also im Allgemeinen zur einfachen Linie, da $\frac{\partial \boldsymbol{V}}{\partial x_{k}}$ weder verschwindet, noch proportional mit $x_{i}$ ausfällt. Der Werth von $R$ zeigt aber, dass

$$
\Sigma \frac{\partial \nabla}{\partial x_{k}} d x_{k}=0 \text {. }
$$

*) Math. Ann. Bd. $\nabla$, S. 292. 
Demnach enthäli $V$ die beiden consecutiven Erzeugenden der Linienfläche. Die Zahl der singulären Erzengonden wird daher durch das Auftreten einer Doppeterzeugenden um vier vermandert.

Sind dagegen $F \Phi \Psi$ von gleichem Grade $m$, so ist $R=2(m-1) x_{k}$, also $\frac{\partial y}{\partial x_{k}} \equiv x_{k}$. Die Doppelerzengende $x$ ist dann selbst Doppellinie des Complexes $V$, welche von den consecutiven Erzeugenden keine in sich enthält*). Die Verminderung der Zahl der singulären Erzengenden bleibt daher die nämliche. Dasselbe gilt auch in dem Falle, wenn einer der Complexe selbst eine Doppellinie besitzt:

Wenn zwei oder drei der erzengenden Complexe die Gerade $x$ zur Doppellinie haben, so wird diese zuglejch vier-, respective sechsfache Linie des Complexes $F$. Die Verminderung der Zahl der singulären Erzeugenden beträgt daher mindestens 16 , resp. 48 , doch scheinen ausserdem noch specielle Beziehungen des Complexes $V$ zu der windschiefen Fläche stattzufinden, deren algebraische Bestimmung schwierig sein dürfte.

In speciellen Fällen können zwei singuläre Erzeugende consecutiv sein. Dazu ist erforderlich, dass mit $V$ zugleich

$$
\left(d x d^{2} x\right)
$$

verschwinde. Daza ist eine Bedingung zwischen den Coefficienten von $F \Phi \psi$ hinreichend, welehe für den Fall $F=a_{x}, \Phi=b_{x}$ das Resultat der Elimination der $x$ aus den vier Gleichungen zweiten Grades:

$$
U_{3}=0, \quad \nabla=0, \quad\left(x^{2}\right)=0, \quad \Psi=0
$$

und den linearen Gleichungen

sein würde.

$$
a_{x}=0, \quad b_{x}=0
$$

Die Fläche wird dagegen developpabel, wenn der Complex $V$ alle den drei Erzeugenden gemeinschaftlichen Geraden in sich exthält. Wir gehen jedoch auf die Betrachtung dieses Falles nicht weiter ein, da er sich besser an die Theorie der Brennflächen anschliesst.

*) In diesem Falle kann nan die erzeugenden Complexe durch irgend drei lineare Combinationen derselben ersetzen. Man erkennt daraus obne weiteres, wovon man sich übrigens auch direct überzeugen kann, dass die Determinante $V$ eine Combinante der drei Formen $F \Phi \psi$ ist.

**) Die am Schluss des $§$ III betrachtete Fläche $\gamma, \Phi, \Psi$ besitzt allerdings nur

$$
4 n p(n+p-2)-6
$$

singuläre Erzeugende. Von diesen haben $2 n p(n+p-2)$ lhre Caspidalpunkte auf der $n \dot{p}+1$-fachen Leitlinie der Fläche. Die Erniedrigrung um sechs (anstatt vier) ist eine Folge daron, dass unter den singulären Erzengenden sick zwei ansgezeichnete befinden, welche doppelt zu zählen sind. 
V.

Die Haupttangenten der windschiefen Fläche.

Die Haupttangenten bilden in Folge der dualistischen Erzeugang der Fläche ein Strahlensystem gleicher Ordnung und Classe. Die Classe desselben ist durch die Zahl der Inflexionen des ebenen Schnittes gegeben, bestimmt sich also yach den Formeln von Plücker durch

$$
3 N(N-2)-6 \dot{D}=6 \operatorname{mn} p[m+n+p-2] .
$$

Man kann diese Zahl auf folgende Weise auch direct bestimmen. Eine Gerade $y$, welche Haupttangente der Fläche ist, muss drei consecutive Erzeugende derselben schneiden, also den Bedingungen

$$
(y x)=0, \quad(y d x)=0, \quad\left(y d^{2} x\right)=0, \quad\left(y^{2}\right)=0
$$

genügen. Wir fragen nach der Zahl der Haupttangenten, welche zwei willkührliche Gerade $a, b$ schneiden, fügen also den Gleichnngen (1) die beiden

hinzu.

$$
a_{y}=0, \quad b_{y}=0
$$

Die Elimination der $y$ liefert die Determinante

$$
\left(\Omega: x d x d^{2} x a b\right)=0 \text {. }
$$

Man elimirirt ans derselben die Differentiale durch zweimalige

\begin{tabular}{|c|c|c|c|c|c|c|c|}
\hline$f^{2}$ & $f \varphi$ & $f \psi$ & \multicolumn{4}{|c|}{$0(m-1) U_{1}$ af $b f$} & \\
\hline$f \varphi$ & $\varphi^{2}$ & $\varphi \psi$ & 0 & $-1)$ & $a \varphi$ & $b \varphi$ & \\
\hline$f \psi$ & $\varphi \psi$ & $\psi^{2}$ & 0 & $-1)$ & $a \psi$ & $b \psi$ & \\
\hline 0 & 0 & 0 & 0 & $V$ & $a_{x}$ & $b_{x}$ & $=0$. \\
\hline-1$)$ & $-1)$ & $-1) U_{3}$ & $V$ & $a$ & 0 & 0 & \\
\hline$a f$ & $a \varphi$ & $a \psi$ & $a_{2}$ & 0 & 0 & 0 & \\
\hline$b f$ & $b \varphi$ & $b \psi$ & $b_{x}$ & 0 & 0 & 0 & \\
\hline
\end{tabular}
Multiplication mit $\Delta=\left(d x^{2}\right)$, wobei sich der bactor $\Delta^{2}$ ohne weiteres wieder absondert und exhält so die Gleichung:

Dasselbe stellt einen Complex $6(m+n+p-3)^{\text {ten }}$ Grades vor, welcher mit den erzeugenden Complexen $F \Phi \psi 12 m n p(n+n+p-3)$ Erzeugende der Linienfläche bestimmt, für welche jedesmal eine der ihnen zugehörigen Haupttangenten $a$ und $b$ schneidet. Man kann dies Resultat auch so aussprechen:

Der Grad der windschiefen Fläche, welche von den Haupttangenten gebildet wird, die einem linearen Complexe angeliören, ist

$$
12 m n p(m+n+p-3) \text {. }
$$

Wenn $a$ und $b$ sich schneiden, so repräsentiren die

$$
12 m n p(m+n+p-3)
$$


Erzeugenden solche Haupttangenten, die entweder in der Ebene $a b$ liegen oder durch den Punkt $a b$ gehen, wonach man wieder die vorhin angeführte Zahl für Classe and Ordnung des Strahlensystems der Haupttangenten erhält.

Die Determinante (4) nimmt eine sehr einfache Gestalt an, wenn einer der Complexe, etwa $F$, linear ist. Alsdann ist $D_{1}=0$. Setzt man noch roraus, dass $a b$ irgend zwei Gerade sind, welche $F$ angehören, so ist

womit sich (4) reducirt auf:

$$
a f=0, \quad b f=0,
$$

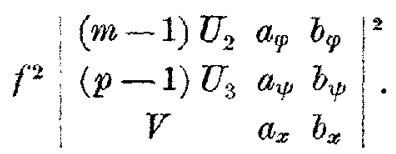

Jede Schnittlinie der vier Complexe $F \Phi \Psi$ und (5) zählt also doppelt. In der That wird, wenn $y$ eine in der Ebene $a b$ liegende Haupttangente der Erzeugenden $x$ ist, die conjugirte Polare von $y$ iu Bezug auf den linearen Complex $F$ ebenfalls Haupttangente von $x$ sein, aber durch den Punkt $a b$ gehen. Es gehören mithin zu jeder Geraden $x$ zwei Haupttangenten.

Wir wenden uns jetzt zur Betrachtung der Haupttangentencurven auf windschiefen Flächen. Wir gehen dabei aus von dem Satze, den Herr Lie zuerst in folgender Form ausgedrückt hat*):

Wenn eine Fläche ihre eigene reciprole Polare hinsichtich sines linearen Complexes ist, so enthält dieselbe eine Haupttangentencurve, deren Tangenten dem Complexe angehören.

Im Anschluss an das Vorige drücken wir diesen Satz so aus: Gehört eine Linienfläche einem linearen Complexe an, so ist die Linienfläche der Haupttangenten, welche ebenfalls Gerade des Complexes sind, developpabel und bildet mit ihrer Rüclikehrcurve eine Haupltangentencurve der ersten Fläche.

Einen liniengeometrischen Beweis dieses Satzes kann man in der folgenden Form geben:

Sind $x, x+d x, x+2 d x+d x^{2}, x+3 d x+3 d x+d^{3} x$ vier consecutive Erzengende der windschiefen Fläche, welche dem Complexe $F=\gamma_{x}$ angehört, so ist:

$$
(\gamma x)=0, \quad(\gamma d x)=0, \quad\left(\gamma d^{2} x\right)=0, \quad\left(\gamma d^{3} x\right)=0 .
$$

Eine Haupttangente $y$ der Fläche, welehe $\gamma$ ebenfalls angehört, genügt den Bedingungen (1), während ausserdem

*) Lie, Partielle Differentialgle"chungen und Complexe, Math. Ann. Bd. V, S. 179. Einen geometrischen Beweis des Lie'schen Satzes gab Herr Klein (Math. Ann. Bd. $V, S, 274$ ). 


$$
(\gamma y)=0
$$

ist. Die consecutive Haupttangente $y+d y$ ist dann bestimmt durch

$$
\begin{gathered}
(x d y)=0, \quad(d y d x)=0, \quad\left(d y d^{2} x\right)+\left(y d^{3} x\right)=0, \quad(\gamma d y)=0, \\
(y d y)=0, \quad(R d y)=0
\end{gathered}
$$

Bildet man jetzt den Ausdruck $\left(a y^{2}\right)$, dessen Verschwinden anzeigt, dass $y$ von $y+d y$ geschnitten wird, so ergiebt sich:

$$
\left(d y^{2}\right)=(\Omega: x d x \gamma k y) \equiv(\Omega: d x \gamma y)=\left|\begin{array}{ccc}
\left(d x^{2}\right) & 0 & 0 \\
0 & \left(\gamma^{2}\right) & 0 \\
0 & 0 & 0
\end{array}\right|=0
$$

$\left(d y^{2}\right)$ verschwindet also in der That, womit der Satz bewiesen ist. Aus dem Lie'schen Satze folgt num unmittelbar unter Berücksichtigung von (4):

Der Rang der algebraischen Haupttangentencurve dex Flache, welche dem linearen Complexe $F=\gamma x$ angehört, ist gleich:

$$
12 p n(p+n-2) \text {. }
$$

Es ist nun leicht, auch Ordnung und Classe dieser Carve zu bestimmen. Da alle Tangenten der Haupttangentencurve dem linearen Complexe angebören, so ist diese eine Complexcurve desselbep. Sie ist daher von gleieher Ordnung und Classe. In der That ist es eine Eigensehaft aller Curven, deren Tangenten Gerade eines linearen Complexes sind, gleiche Ordnung und Classe zu besitzen. Es sei nämlich $N$ die Ordnung der Complexcurve. In einer willkührlichen Eibene $e$ liegen dann $N$ Curvenpunkte. Die Schmiegungsebenen derselben sind aber Ebenen des linearen Complexes, gehen daher dürch einen in $e$ gelegenen Punkt, nämlich denjenigen, welcher durch den linearen Complex der Ebene $e$ zugeordnet ist.

Betrachtet man jetzt die Haupttangentencurve als von den Centren solcher Büschel gebildet, deren Strahlen zugleich Tangenten der Fläche und Linien des Complexes $\gamma$ sind, so ergiebt sich:

In jeder Ebene liegen so viel Punkte der Haupttangentencurve als Tangenten der Fläche vorhanden sind, die dem linearen Complexe angehören und einen Punkt der Haupttangentencurve exthalten. Die Zahl dieser Punkte ist daher im Allgemeinen gleich der Classe des eberen Sehnittes der Näche ${ }^{*}$, Ordnung und Classe der Haupttangentencurve ist daher im Allgemeinen dureh den Rang der Fläche gegeben, d. h. gleich

$$
2 n p(n+p-1) \text {. }
$$

*) Es ist dabei zo beachten, dass die Zahl der Tangenten an die Schnittcurve von einem nicht willkührlich zu wählenden Punkte aus zu bestimmen ist. In besonderen tällen kann daher die Ordnung der Haupttangentencurve auch kleiner sein als die Classe des ebenen Schnittes. 
Eine Complexcurve kann ferner im Allgemeinen keine stationären Ebenen enthalten. Denn es müssen dann drei consecutive Tangenten derselben in einer Ebene liegen, was vermöge der Erzeuguug der Curve nur dann möglich ist, wenn sie zugleich durch einen Punkt geher. Aus dem dualistischen Charakter der Complexcurve folgt dann, dass sie auch keine Spitzen enthält.

Insbesondere für den vorliegenden Fall kann man sich von der der letzteren Behauptung auf folgende Weise überzengen. Auf jeder Erzeugenden $x$ liegen zwei Punkte der Haupttangentencurve, die Schnittpunkte von $x$ mit den beiden Geraden $y$, welche durch die Gletchungen (1) and $\gamma y=0$ bestimmt sind. Für eine Spitze der Haupttangentencurve müssten jedenfalls diese beiden Punkte coincidiren, $d$. h. die Gleichungen eine Doppelwurzel $y$ besitzen. Die letztere Bedingung führt auf

$$
\left(\Omega: x d x d^{2} x y\right)=0,
$$

d. h. auf die singulären Erzeugenden der Fläche.

Wir weisen jetzt nach, dass die Haupttangentencurve in den Cuspidalpunkten der Fläche stationäre Tangenten besitzt.

Dazu betrachten wir zunächst die Zahl der Punkte, welche die Haupttangentencurve mit einer dureh die Generatrix a gehenden Ebene $e$ gemein hat. Bezeichnet $\nu$ den Grad der Fläche, $d$ ihre Doppelcurve, so ist die in der Ebene $e$ liegende Schnittcurve eine Curve $\nu-1^{\text {ter }}$ Ordnung mit $d-(n-2)$ Doppelpunkten. Die Zahl der Tangenten, welche sich von dem auf a liegenden Centrum des in $e$ befindlichen Strahlbüschels ziehen lassen, ist:

$$
(\nu-1)(\nu-2)-2 a+2(n-2) \text {. }
$$

Rechnet man die beiden auf $a$ liegenden Punkte der Haupttangentencurve hinzu, so ist die Anzahl der Punkte derselben in der Ebene $e$

$$
n(n-1)-2 d \text {, }
$$

wie es sein muss. Es sei jetzt $a$ eine singuläre Erzengende; die Schnittcurve berührt dieselbe im Cuspidalpunkte der Fläche. Es existiren daher in der Ebene $e$

$$
(n-1)(n-2)-2 d+2(n-2)-1=n(n-1)-2 d-3
$$

nicht auf $a$ gelegene Punkte der Haupttangentencurve.

Daher liegen auf a im Cuspidalpunte drei zusammenfallende weitere Punlte derselben, d. h. die singuläre Erzeugende ist stationäre Tangente im Cuspidalpunkte.

Fallt endlich die schneidende Ebene e mit der parabolischen Ebene der Fläche zusammen, so ist die Schnitteurve vom $n-2^{\text {ten }}$ Grade mit $a-2(n-2)$ Doppelpunkten, und berührt $a$ im Cuspidalpunkte. Da ferner das Centrum des Strahlbüschels in der Ebene $e$ mit dem letzteren zusammenfält, so ist die Zahl der Tangenten an die Curve: 


$$
(n-2)(n-3)-2 d+4(n-2)-2=n(n-1)-2 d-4 \text {. }
$$

Die parabolische Ebene enthält daher vier zusammenfallende Punkte der Haupttangentencurve, sie ist Schmiegungsebene derselben.

Man kann diesen Betrachtungen noch die Bemerkung hinzufügen, dass auf einer Doppelerzeugenden 4 Punkte der Haupttangentencurve liegen. Für eine Rückkehrgeneratrix rücken dieselben paarweise zusammen, dieselbe ist Doppeltangente der fraglichen Curve.

Aus den angestellten Betrachtungen ergiebt sich nun:

Die Haupttangentencurve besitzt $4 n p(n+p-2)$ stationäre Tangenten, welche mit den singuläron Exzeugenden dej. Fläche zusammenfallen. Wir werden weiterhin zeigen, dass ausserdem noch andere stationäre Tangenten vorhanden sind.

Wir weisen jetzt nach, wie man die Ordnung und Classe der Haupttangentencurve auf analytischem Wege bestimmen kann.

Ess sei $x$ eine Erzeugende der Fläche, $y$ eine ihr zugeordnete Haupttangente, für welche die Gleichungen (1) bestehen. Die Ebene $x y$ ist dann zugleich die Schmiegungsebene der Haupttangentencurve, deren Tangente $y$ ist. Ein willkührlicher Strahl des Büschels $x y$ ist ferner $x+\lambda y$. Damit derselbe zwei willkührliche Gerade $a b$ schneide $_{\curlywedge}$ müssen die Gleichungen

$$
\begin{aligned}
& a_{x}+\lambda a_{y}=0 \\
& b_{x}+\lambda b_{y}=0
\end{aligned}
$$

bestehen. Aus denselben folgt:

$$
a_{x} b_{y}-b_{x} a_{y}=0 \text {. }
$$

Soll ausserdem $y$ dem linearen Complexe $F=\gamma x$ angehören, so ist

$$
\gamma y=0 \text {. }
$$

Die Elimination der $y$ aus den Gleichungen (1), (6), (7) liefert

$$
\left(\Omega: x d x d^{2} x \gamma \quad a_{x} b-b_{x} a\right)=0 \text {. }
$$

Zieht man von der siebenten Horizontal- oder Vertikalreihe der Determinante (8) die Summe der mit $x_{1} x_{2} \cdots x_{6}$ multiplicirten ersten sechs analogen Reihen ab, so sondert sich aus (8) der Factor. $\left(d x^{2}\right)\left(d x^{2}\right)$ aus, welcher dèn Forderungen des Problems fremd ist. Darnach geht (8) über in:

$$
\left(\Omega: d x \gamma \quad a_{x} b-b_{x} a\right)=0 .
$$
mit $\Delta^{2}$

Man entfernt aus (9) endlich die Differentiale durcb Multiplication und erhält so:

$$
\Delta=(d x \gamma \varphi \psi x k)
$$




$$
\left|\begin{array}{ccccc}
\gamma^{2} & \gamma \varphi & \gamma \psi & \gamma^{2} & p_{1} \\
\gamma \varphi & \varphi^{2} & \varphi \psi & \varphi \gamma & p_{2} \\
\gamma \psi & \varphi \psi & \psi^{2} & \psi \gamma & p_{3} \\
\gamma^{2} & \varphi \gamma & \psi \gamma & 0 & 0 \\
p_{1} & p_{2} & p_{3} & 0 & 0
\end{array}\right|=0
$$

wo zur Ablürzong gesetzt ist:

$$
\begin{aligned}
& p_{1}=a_{x} b_{y}-b_{x} a_{y}, \\
& p_{2}=a_{x} b_{\varphi}-b_{x} a_{\varphi}, \\
& p_{3}=a_{x} b_{\psi}-b_{x} a_{\psi} .
\end{aligned}
$$

Man kann endlich noch annehmen, dass $a, b$ zwei Gerade des Complexes $\gamma_{x}$ sind, wodurch die Gleichung (10) übergeht in :

$$
\left|\begin{array}{ccc}
\varphi^{2} & \varphi \psi & p_{2} \\
\varphi \psi & \psi^{2} & p_{3} \\
p_{2} & p_{3} & 0
\end{array}\right|=0 \text {. }
$$

(11) repräsentirt einen Complex rom Grade

$$
2(m+n-1) \text {, }
$$

welcher $4 m n(m+n-1)$ Erzengende der Fläche bestimmt. Wenn man $a, b$ als zwei sich schneidende Geraden voraussetzt, so liegt entweder ein Punkt der Haupttangentencurve in der Ebene $(a b)$ oder eine Schmiegungsebene derselben enthält den Punkt $(a b)$. Die Aṇzahl beider Falle ist daher $2 m n(n+n-1)$, wie schon oben gefunden wurde.

Man übersieht leicht, dass die gegebenen Zahlen für Rang, Classe, Ordnung und stationäre Tangenten der Haupttangentencurve auch dann noch bestehen bleiben, wenn die Fläche zwei linearen Complexen angehört, an deren Stelle man zwei specielle substituiren kann. Dem Büschel linearer Complexe $\alpha_{x}+\lambda \beta_{x}=0$ entsprechend erhät man dann alle Haupttangentencurven der Fläche. Sie sind daher algebraische Curven, deren charalteristische Zahlen die folgenden sind*):

$$
\begin{aligned}
& \text { Rang }=12 m(m-1), \\
& \text { Classe } \\
& \text { Ordnung }\}=2 m^{2}, \\
& \text { stationäre Tangenten } \left.4 m(m-1)^{* *}\right) .
\end{aligned}
$$

Diese Zahlen modificiren sich aber erheblich, wenn die Fläche zwei linearen Complexen, die eine specielle Congruenz gemein haben, angehört. Als erzengende Complexe wählen wir dann den speciellen $\alpha_{x}=0$, den allgemeinen $\beta_{x}=0$, wobei $(\beta \alpha)=0$ und $\Psi=0$. Aus

*) Vgl. eine Bemerkung von Herrn Klein (Math. Ann. Bd. V, S. 23), sowie die schon vorher citirte Arbeit von Lie (daselbst S. 179).

*) In die Zahl $4 m(m-1)$ sind die anderweitigen stationären Tangenten nicht eingerechnet. Vgl. \& VII. 
der Determinante (4) ergiebt sich durch Substitation der gehörigen Grössen unmittelbar zur Bestimmung des Ranges derjenigen Haupttangentencurve, welche dem Complexe $\alpha_{x}+\lambda \beta_{x}=0$ angehört:

\begin{tabular}{|c|c|c|c|c|c|c|c|}
\hline .0 & 0 & $\alpha \psi$ & 0 & 0 & 0 & $b \alpha$ & \\
\hline 0 & $\beta^{2}$ & $\beta \psi$ & 0 & 0 & $\lambda \beta^{2}$ & $b \beta$ & \\
\hline$\alpha \psi$ & $\beta \psi$ & $\psi^{2}$ & $0(z$ & -1) $U_{3}$ & $\theta$ & $b \psi$ & \\
\hline 0 & 0 & 0 & 0 & $V$ & 0 & $b \dot{x}$ & $=0$ \\
\hline 0 & 0 & -1) $U_{3}$ & $V$ & 0 & 0 & 0 & \\
\hline 0 & $\lambda \beta^{2}$ & $\theta$ & 0 & 0 & 0 & 0 & \\
\hline$b a$ & $b \beta$ & $b \psi$ & $b x$ & 0 & 0 & 0 & \\
\hline
\end{tabular}

Dabei ist $\Theta=\alpha \psi+\lambda \beta \psi$ gesetzt. Wir schreiben (12) in der Form: (13)

$$
A_{11} U_{3}{ }^{2}(p-1)^{2}+2(p-1) U_{3} V A_{12}+A_{22} V^{2}=0 \text {. }
$$

Man sieht nun safort, dass $A_{11}=0$, während

$$
A_{12} \equiv(b \alpha) \lambda^{2}\left(\beta^{2}\right)\left(\beta^{2}\right)(\alpha \psi) b x \text {. }
$$

Da auch $V \equiv(\alpha \psi)^{2}\left(\beta^{2}\right)$, so sondert sich aus der Gleichung (13) der Factor $(\alpha \psi)^{3}$ aus, welcher sich auf die singulären Linien bezieht und der Frage fremd ist. Der Rang der algebraischen Haupttangentencurven ist daher:

$$
6 \rho(p-1) \text {. }
$$

Um die Ordnung, respective Classe derselben zu bestimmen, sind in (10) die gehörigen Werthe zu substibuiren. Es entsteht dann:

$$
\left|\begin{array}{ccccc}
0 & 0 & \kappa \psi & 0 & p_{1} \\
0 & \beta^{2} & \beta \psi & \lambda \beta^{2} & p_{2} \\
\alpha \psi & \beta \psi & \psi^{2} & \Theta & p_{3} \\
0 & \lambda \beta^{2} & \Theta & 0 & 0 \\
p_{1} & p_{2} & p_{3} & 0 & 0
\end{array}\right|=0 .
$$

Aber aus dieser Gleichung sondert sich der lineare Factor $p_{1}$ ab. Daher ist die Ordnung und Classe der Hauptangentencurven gleich

$$
p(2 p-1) \text {. }
$$

Man kann dies letztere Resultat auch geometrisch wie folgt begründen. Die ebene Schnittcurve der Fläche ist eine Curve $2 p^{\text {ter }}$ Ordnung mit zwei unendlich nahen $p$-facben Punkten, also von der Classe $2 p^{2}$. Eine Gerade des Strahlbüschels, mit dem Centrum 0 , welches der Complex $\alpha_{x}+\lambda \beta_{x}=0$ in der Ebene der Schnittcurve bestimmt, berührt aber diese Curve in dem $p(+p)$-fachen Punkte, es giebt also noch*)

*) Vgl. Cayley, Second Memoir on skew surfaces, otherwise scrolls Phil. Trans. 1864, p. 559. Ueber die Bezeichnung $p(+q)$-facher Punkt vergl daselbst p. 56t, sowie Cremona, Theorie der Oberfächen S. 59. 


$$
2 p^{2}-p=p(2 p-1)
$$

weitere Tangenten, welche von $O$ auslaufen, auf denen je ein Punkt der durch $\alpha_{x}+\lambda \beta_{x}=0$ bestimmten Haupttangenteneurve liegt.

Wir figen endlich noch die Bemerkung hinzu, dass die Hauptangentencurven dieser speciellen windschiefen Flächen in den Cuspidalpunkten derselben keine stationären Tangenten haben. Die Schmiegungsebenen der Haupttangentencurve nämlich, welche durch einen auf $\alpha$ gelegenen Punkt $A$ hindurchgehen, erhält man durch die Schnittpunkte der durch $A$ gehenden durch den betreffenden Complex zugeordneten Ebene, welche den Strahl $\alpha$ in sich enthält. Die $2 p(p-1)$ auf $\alpha$ liegenden Cuspidalpunkte der Fläche, durch welche jede Haupttangentencurve hindurchgeht, müssen also als Punkte zählen, in denen dieselbe wirkliche Schmiegungsebenen besitzt.

Die Zahl der anderweitigen stationären Tangenten der Haupttangentencurve werden wir im folgenden $\$$ bestimmen.

Wir schliessen hieran die folgende Betrachtung. Wenn eine windschiefe Fläche einem linearen Complexe nicht angehört, so besitzt sie nur $2 m n p$ discrete Tangentenebenen, welche einem willkübrlichen linearen Complexe angehören, d. h. für welche jede durch den Berührungspunkt gezogene Flächentangente zugleich Complexgerade ist. Ein Büschel linearer Complexe giebt erst wieder zu einer Curve auf der Fläche Veranlassung, deren Puakte die Berührungspunkte solcher Ebenen sind, die Complexen derselben in dem eben bezeichneten Sinne angehören. Es ist leicht, die Ordnung einer derartigen Curve zu bestimmen.

Es sei das Büschel linearer Complexe

$$
a_{x}+\lambda b_{x}=0 \text {. }
$$

Damit die Erzeugende $x$ und die ihr zugehörige Haupttangente $y$ Geraden eines der Complexe (15) seien, muss

$$
\begin{aligned}
& a_{x}+\lambda b_{x}=0, \\
& a_{y}+\lambda b_{y}=0
\end{aligned}
$$

sein. Darans folgt wieder Gleichung (6). Damit ferner ein willkührlicher Strahl des Büschels $(x y)$ die beiden beliebigen Geraden $a, \beta$ schneide, ist

$$
\alpha_{x} \beta_{y}-\alpha_{y} \beta_{x}=0
$$

zu setzen. Die Elimination der $y$ aas (1), (6), (16) liefert die Relation:

oder

$$
\left(\Omega: x d x d^{2} x \quad b_{x} a-a_{x} b \quad \beta \alpha-\alpha_{x} \beta\right)=0 \text {, }
$$

$$
\left(\Omega: d x \quad b_{x} a-a_{x} b \quad \beta_{x} \alpha-\alpha \beta\right)=0,
$$

oder endlich durch Maltiplication mit $\Delta^{2}$ : 
wo

$$
\left\{\begin{array}{l}
p_{1}=b_{x} a_{f}-a_{x} b_{f}, \quad q_{1}=\beta_{x} \alpha_{f}-\alpha_{x} \beta_{f}, \\
p_{2}=b_{x} a_{\varphi}-a_{x} b_{\varphi}, \quad q_{2}=\beta_{x} \alpha_{\varphi}-\alpha_{x} \beta_{\varphi}, \\
p_{3}=b_{x} a_{\psi}-a_{x} b_{\psi}, \quad q_{3}=\beta_{x} \alpha_{\psi}-\alpha_{x} \beta_{\psi} .
\end{array}\right.
$$

Die Determinante (17) ist daher vom Grade $2(n+n+p-1)$ und liefert als Grad der fraglichen Curve

$$
2 m n p(n+n+p-1) \text {. }
$$

VI.

Die Curve vierpunktiger Berïhrung auf den windschiefen Flächen.

Eine jede windschiefe Fläche besitzt eine Curve vierpunktiger Berührung, welche, soviel mir bekannt, bisher nicht Gegenstand der Untersuchung gewesen ist. Je vier consecutive Erzeugende bestimmen zwei Gerade, welche sie schneiden, d. h. zwei im Allgemeinen discrete vierpunktige Tangenten, und damit zwei Punkte der Curve. Wenn dieselben coincidiren, so berührt die Curve die betreffende Frzeugende. Wir werden im Folgenden den Grad derselben, sowie die Zahl ihrer Berührungen mit den Erzengenden bestimmen und dabei diejenigen Eliminationen vollständig durehführen, zu denen dieses Problem Veranlassung giebt.

Eine Gerade $y$, welche vier consecutive Erzeugende

$$
x, \quad x+d x, \quad x+2 d x+d^{2} x, \quad x+3 d x+3 d^{2} x+d^{3} x
$$

schneidet, erfüllt die Bedingungen:

$$
0=y x_{i}^{2}, \quad 0=(y d x), \quad 0=\left(y d^{2} x\right), \quad 0=\left(y d^{3} x\right), \quad 0=\left(y^{2}\right) .
$$

Sie bestimmt mit $x$ zwei ebene Strahlbüschel $y+\lambda x$. Verlangt man, dass ein Strahl derselben zwei willkührliche Gerade schneide, welche wir $b, a$ nennen, so ist

$$
\left(a_{y} b_{x}-b_{x} a_{y}\right)=0
$$

Die Elimination der $y$ liefert die Gleichung:

$$
\left(\Omega: x d x d^{2} x d^{3} x \quad a_{x} b-b_{x} a\right)=0 \text {. }
$$

Aus derselben sind die Differentiale zu eliminiren. Es gelingt dies für die ersten und zweiten Differentiale $d x, d^{2} x$ sofort durch Multiplication mit $\Delta^{2}$, wobei sich wie oben $\Delta^{2}$ wieder als Factor aussondert. 
A. Voss.

-Zugleich wird dabei der unwesentliche Factor $(k x)^{4}$ entfernt, dessen Coefficienten $k$ die homogenen Differentiale verbinden, so dass

$$
\begin{aligned}
& k d x=0, \\
& k d^{2} x=0, \\
& k d^{3} x=0 .
\end{aligned}
$$

Man erhält so in Räcksicht auf II. (10):

$$
\mid \begin{array}{ccccccc}
f^{2} & f \varphi & f \psi & 0 & (m-1) U_{1} f d^{3} x p_{1} \\
f \varphi & \varphi^{2} & \varphi \psi & 0 & (n-1) U_{2} \varphi d^{3} x p_{2} \\
f \psi & \varphi \psi & \psi^{2} & 0 & (p-1) U_{3} \psi d^{3} x p_{3} \\
0 & 0 & 0 & 0 & V & x d^{3} x & 0 \\
(m-1) U_{1} & (n-1) U_{2} & (p-1) U_{3} & V & 0 & 0 & 0 \\
f d^{3} x & \varphi d^{3} x & \psi d^{3} x & x d^{3} x & 0 & 0 & 0 \\
p_{1} & p_{2} & p_{3} & 0 & 0 & 0 & 0
\end{array}
$$

Die $p_{1} p_{2} p_{3}$ haben dabei die in $\mathrm{I}$. (18) angegebene Bedeutung. Um nun aus (1) noch die dritten Differentiale zu entfernen, verfahren wir folgendermassen. Zunächst ergiebt sich durch Differentiation der Gleichungen II. (10):

$$
\left\{\begin{array}{l}
f_{i} d^{3} x_{i}+(m-1) f_{i k} d x_{k} d^{2} x_{i}=(m-1)(k x)^{2} \frac{\partial U_{1}}{\partial x_{2}} d x_{i} \\
\varphi_{i} d^{3} x_{i}+(n-1) \varphi_{i k} d x_{k} d^{2} x_{i}=(n-1)(k x)^{2} \frac{\partial U_{2}}{\partial x_{i}} d x_{i} \\
\psi_{i} d^{3} x_{i}+(p-1) \psi_{i k} d x_{k} d^{2} x_{i}=(p-1)(k x)^{2} \frac{\partial U_{3}}{\partial x_{i}} d x_{i}
\end{array}\right.
$$

Ferner ist

$$
\left\{\begin{aligned}
\left(x d^{3} x\right) & =-3\left(d x d^{2} x\right) \\
2\left(d x d^{2} x\right) & =-(k x)^{2} \frac{\partial V}{\partial x_{i}} d x_{i}
\end{aligned}\right.
$$

Um aus (2) noch die zweiten Differentiale zu entfernen, setzen wir:

$$
\left\{\begin{array}{l}
f_{i k} d x_{k} d x_{i}^{2}=\Pi_{1} \\
f_{i} d^{2} x_{i}=(m-1) U_{1}(k x)^{2} \\
\varphi_{i} d^{2} x_{i}=(n-1) U_{2}(k x)^{2} \\
\psi_{i} d^{2} x_{i}=(p-1) U_{3}(k x)^{2} \\
x_{i} d^{2} x_{i}=(k x)^{2} V \\
k_{i} d^{2} x_{i}=0 \\
d x_{i} d^{2} x_{i}=\Sigma
\end{array}\right.
$$

Die Determinante der sieben Gleichungen (4) muss versehwinden. Wir schreiben sie in der Gestalt: 


$$
-\Pi_{1} A_{1}-A_{2}(k x)^{2}-(k x)^{2} V A_{3}-\Sigma A_{4}=0 .
$$

Die Grössen $A$ haben in (5) die folgenden Werthe. Die Unterdeterminante $A_{1}$ ist offenbar gleich $-\left(d x^{2}\right)=(k x)^{2} V$. Der Werth von $A_{2}$ ist gegeben durch:

$$
A_{2}=\left[\begin{array}{cc}
f_{i k} d x_{k} & 0 \\
f_{i} & (m-1) U_{1} \\
\varphi_{i} & (n-1) U_{2} \\
\psi_{i} & (p-1) U_{3} \\
x_{i} & 0 \\
k_{i} & 0 \\
d x_{i} & 0
\end{array} .\right.
$$

In der Determinante rechts ist dabei von den sechs ersten nur die Verticalreihe mit dem Index $i$ hingeschrieben. Man erbält nun sofort:

$\Delta A_{2}=\left[\begin{array}{ccccccc}-U_{1}(k x)^{2} & f_{i} f_{i k} d x_{k} & \varphi_{i} f_{2 k} d x_{k} & \psi_{i} f_{i k} \alpha x_{k} & 0 & a_{1} & 0 \\ 0 & f^{2} & f \varphi & f \psi & 0 & f k(m-1) U_{1} \\ 0 & f \varphi & \varphi^{2} & \varphi \psi & 0 & 0 k_{i}(n-1) U_{2} \\ 0 & f \psi & \varphi \psi & \psi^{2} & 0 & 0 & \\ 0 & 0 & 0 & 0 & 0 & k x & 0 \\ 0 & k f & k \varphi & k \psi & k x k_{k}^{2} & 0 \\ (d x)^{2} & 0 & 0 & 0 & 0 & 0 & 0\end{array}\right.$.

wo zur Abkürzung gesëtzt ist:

Darnach ist:

$$
a_{1}=f_{i k} d x_{k} k_{k}
$$

$$
A_{2} \dot{\Delta}=-\Delta(k x)^{2}\left|\begin{array}{cccc}
f_{i} f_{i k} d x_{k} & \varphi_{i} f_{i k} d x_{k} & \psi_{i} f_{i k} d x_{k} & 0 \\
f^{2} & f \varphi & f \psi & (n-1) U_{1} \\
f \varphi^{-} & \varphi^{2} & \varphi \psi & (n-1) U_{2} \\
f \psi & \varphi \psi & \psi^{2} & (p-1) U_{3}
\end{array}\right|
$$

Setzt man zur Abkürzung:

$$
A_{2}=-(k x)^{2} \Omega_{k}^{\prime} d x_{k}
$$

so besteht, wie man sofort aus der Betrachtung ron $\Omega_{k}^{\prime} d x_{k}$ ersieht, die Beziehung:

$$
\Omega_{k}^{\prime} x_{k}=(m-1) U_{1} V
$$

Es ist ferner:

$$
A_{4}=f_{i k} d x_{i} d x_{k}=-(k x)^{2} U_{1} .
$$

Endlich hat man: 


$$
A_{3}=(k x)^{2}\left|\begin{array}{lllllllll}
f_{11} & f_{12} & f_{13} & f_{14} & f_{15} & f_{16} & \varphi_{1} & \psi_{1} & x_{1} \\
f_{21} & f_{22} & f_{23} & f_{24} & f_{25} & f_{26} & \varphi_{2} & \psi_{2} & x_{2} \\
f_{31} & f_{32} & f_{33} & f_{34} & f_{35} & f_{36} & \varphi_{3} & \psi_{3} & x_{3} \\
f_{41} & f_{42} & f_{43} & f_{44} & f_{45} & f_{46} & \varphi_{4} & \psi_{4} & x_{4} \\
f_{51} & f_{52} & f_{53} & f_{54} & f_{55} & f_{56} & \varphi_{5} & \psi_{5} & x_{5} \\
f_{61} & f_{62} & f_{63} & f_{64} & f_{65} & f_{66} & \varphi_{6} & \psi_{6} & x_{6} \\
\varphi_{1} & \varphi_{2} & \varphi_{3} & \varphi_{4} & \varphi_{5} & \varphi_{6} & 0 & 0 & 0 \\
\psi_{1} & \psi_{2} & \psi_{3} & \psi_{4} & \psi_{5} & \psi_{6} & 0 & 0 & 0 \\
d x_{1} & d x_{2} & d x_{3} & d x_{4} & d x_{5} & d x_{6} & 0 & 0 & 0
\end{array}\right| .
$$

Setzt man daher:

$$
A_{3}=(k x)^{2} \theta_{i}^{\prime} d x_{i}
$$

so ist

$$
U_{1}=\Theta_{i}^{\prime} x_{i}
$$

Aus der Gleichung (5), welche jetzt die folgende Gestalt angenommen hat:

$$
\Pi^{\prime} V=\Sigma U_{1}-\left(k_{k} x\right)^{2} V\left(\Theta_{i}^{\prime} d x_{i}\right)+(k x)^{2}\left(\Omega_{k}^{\prime} d x_{k}\right)
$$

hat man nun für die erste der Gleichungen (2) den folgenden Ausdruck:

$$
\frac{V}{(n-1)} f_{i} d^{3} x_{i}=-\Sigma U_{1}+(k x)^{2} V\left(\Theta_{i}^{\prime} d x_{i}\right)-(k x)^{2}\left(\Omega_{i}^{\prime} d x_{k}\right)+(k x)^{2} \nabla^{\hat{\partial} U_{i}} d x_{i}
$$
und dem analog:

$$
\begin{aligned}
& \frac{V}{(n-1)} \varphi_{i} d^{3} x_{i}=-\Sigma U_{2}+(k x)^{2}\left(\Theta_{i}^{\prime \prime} d x_{i}\right) V-(k x)^{2}\left(\Omega_{k}^{\prime \prime} d x_{k}\right)+(k x)^{2} V \frac{\partial U_{2}}{\partial x_{i}} d x_{i}, \\
& \frac{V}{(p-1)} \psi_{i} d^{3} x_{i}=-\Sigma U_{3}+(k x)^{2}\left(\Theta_{i}^{\prime \prime \prime} d x_{i}\right) V-(k x)^{2}\left(\Omega_{k}^{\prime \prime \prime} d x_{k}\right)+(k x)^{2} V_{\frac{\partial}{\partial} x_{i}} d x_{i}
\end{aligned}
$$

Dabei bedeuten $\theta^{\prime \prime}, \Theta^{\prime \prime \prime}, \Omega^{\prime \prime}, \Omega^{\prime \prime}$ diejenigen Ausdrücke, in welche $\Theta^{\prime}, \Omega^{\prime}$ durch Vertausehung von $f$ mit $\varphi$, respective $\psi$ übergehen.

Man hat daher ferner:

$$
\frac{V}{(k x)^{2}}\left(\frac{U_{2}}{m-1} f_{i} d^{3} x_{2}-\frac{U_{1}}{n-1} \varphi_{i} d^{3} x_{i}\right)=-V\left|\begin{array}{l}
U_{1} \Theta_{i}^{\prime} d x_{i} \\
U_{2} \Theta_{i}^{\prime \prime} d x_{i}
\end{array}\right|-\mid \begin{gathered}
U_{1}\left(\frac{\partial U_{1}}{\partial x_{i}}-\Omega_{i}^{\prime}\right) d x_{i} \\
U_{2}\left(V^{\partial} \frac{\partial U_{2}}{\partial x_{i}}-\Omega_{i}^{\prime \prime}\right) d x_{i}
\end{gathered}
$$

In dieser Gleichung ist nach II. (7) und VI. (10):

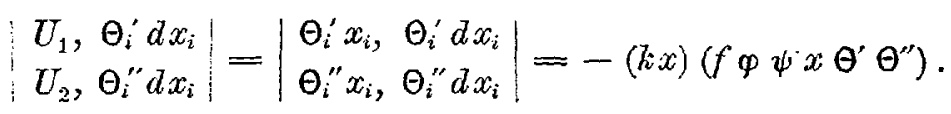

Es ist ferner:

$$
[2(m+n+p)-7] U_{1} \nabla=V\left(\frac{\partial U_{i}}{\partial x_{i}} x_{i}\right)-(m-1) U_{1} \nabla
$$

Sonach mit Berücksichtigung von II. (7) und VI. (7): 


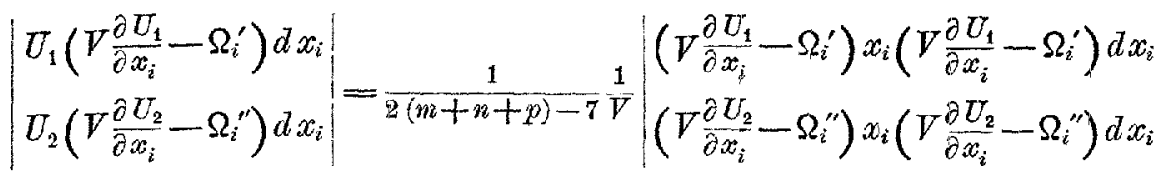

$$
\begin{aligned}
& =\frac{-(h x)}{V(2(m+n+p)-7)}\left(f \varphi \psi x V^{\prime} \frac{\partial U_{1}}{\partial x}-\Omega^{\prime} \quad V^{\frac{\partial U_{z}}{\hat{p} x}-\Omega^{\prime \prime}}\right) \text {. }
\end{aligned}
$$

Wir haben demnach endlich die folgenden Gleichungen:

$$
\left\{\begin{array}{l}
\frac{(n-1) U_{2} f_{i} d^{3} x_{i}-(m-1) U_{1} \varphi_{i} d^{3} x_{i}}{(n-1)(m-1)(k x)^{3}} \\
=\left(f, \varphi, \psi, x, \Theta^{\prime}, \Theta^{\prime \prime}\right)+\frac{1}{V^{2}} \frac{1}{2(m+n+p)-7}\left(f, \varphi, \psi, x, V \frac{\partial U_{1}}{\partial x}-\Omega^{\prime}, \nabla \frac{\partial U_{2}}{\partial x}-\Omega^{\prime \prime}\right), \\
\frac{(m-1) U_{1} \psi_{i} d^{3} x_{i}-(p-1) U_{3} f_{i} d^{3} x_{i}}{(m-1)(p-1)(k x)^{3}} \\
=\left(f, \varphi, \psi, x, \Theta^{\prime \prime \prime}, \Theta^{\prime}\right)+\frac{1}{V^{2}} \frac{1}{2(m+n+p)-7}\left(f, \varphi, \psi, x, V \frac{\partial U_{3}}{\partial x}-\Omega^{\prime \prime}, V \frac{\partial U_{1}}{\partial x}-\Omega^{\prime}\right), \\
\frac{(p-1) U_{3} \varphi_{i} d^{3} x_{i}-(n-1) U_{2} \psi_{i} d^{3} x_{i}}{(p-1)(n-1)(k, x)^{3}} \\
=\left(f, \varphi, \psi, x, \Theta^{\prime \prime}, \Theta^{\prime \prime}\right)+\frac{1}{V^{2}} \frac{1}{2(m+n+p)-7}\left(f, \varphi, \psi, x, V^{\partial U_{2}} \frac{1}{\partial x}-\Omega^{\prime \prime}, V^{\partial} \frac{U_{3}}{\partial x}-\Omega^{\prime \prime \prime}\right) .
\end{array}\right.
$$

Stellt man dagegen zu der Gleichung (11) noch den folgenden Ausdruck:

$$
x_{i} d^{3} x_{i}=-\Sigma+\frac{\partial V}{\partial x_{i}} d x_{i}
$$

so ist

$$
\begin{aligned}
& \frac{V f_{i} d^{3} x_{i}-(m-1) U_{1}\left(x_{i} d^{3} x_{i}\right)}{(k x)^{2}(n-1)}=\left(V \Theta_{i}^{\prime}-\Omega_{i}^{\prime}+V_{\partial U_{1}}^{\partial x_{i}}\right) d x_{i}-d x_{i} \frac{\partial V}{\partial x_{i}} U_{1} \\
& =\left|\begin{array}{cc}
V & \frac{\partial V}{\partial x_{i}} d x_{i} \\
U_{1}\left(\Theta_{i}^{\prime}+\frac{\partial U_{1}}{\partial x_{i}}-\frac{\Omega_{i}^{\prime}}{V}\right) d x_{i}
\end{array}\right| .
\end{aligned}
$$

Schreibt man die letzte Determinante endlich in der Gestalt:

$$
\frac{1}{2(m+n+p)-3}\left|\begin{array}{ll}
\frac{\partial V}{\partial x_{i}} x_{i} & \frac{\partial V}{\partial x_{i}} d x_{i} \\
\left(\Theta_{i}^{\prime}+\frac{\partial U_{i}}{\partial x_{i}}-\frac{\Omega_{i}^{\prime}}{V}\right) x_{i} & \left(\Theta_{i}^{\prime}+\frac{\partial U_{i}}{\partial x_{i}}-\frac{\Omega_{i}^{\prime}}{V}\right) d x_{i}
\end{array}\right|,
$$

so ergiebt sich: 
14)

$$
\left\{\begin{array}{c}
\frac{V f_{i} d^{3} x_{i}-m-i U_{1}\left(x_{i} d^{3} x_{i}\right)}{(k x)^{3}(m-1)} \\
=\frac{-1}{2(m+n+p)-3}\left(f \varphi \psi x \frac{\partial V}{\partial x_{i}}, \theta_{i}^{\prime}+\frac{\partial U_{1}}{\partial x_{i}}-\frac{\Omega_{i}^{*}}{V_{i}}\right) \\
\frac{V \varphi_{i} d^{3} x_{i}-n-1 U_{2}\left(x_{i} d^{3} x_{i}\right)}{(k x)^{3}(n-1)} \\
=\frac{-1}{2(m+n+p)-3}\left(f^{*} \varphi \psi x \frac{\partial V}{\partial x_{i}}, \theta_{i}^{\prime \prime}+\frac{\partial U_{2}}{\partial x_{i}}-\frac{\Omega_{i}^{\prime \prime}}{V}\right), \\
\frac{V \psi_{i} d^{3} x_{i}-p-1 U_{3}\left(x_{i} d^{3} x_{i}\right)}{(k x)^{3}(p-1)}-\frac{1}{2(m+n+p)-3}\left(f \varphi \psi x \frac{\partial V}{\partial x_{i}}, \theta_{i}{ }^{\prime \prime}+\frac{\partial U_{3}}{\partial x_{i}}-\frac{\Omega_{i}^{\prime \prime}}{V}\right) .
\end{array}\right.
$$

Die Ausdrücke rechterhand in den Gleichungen (14) enthalten, wie noch bemerkt werden mag, nur scheinbar den Nenner $V$. Denn die Determinante

$$
\left(f \varphi \psi x \Omega_{i}^{\prime} \frac{\partial V}{\partial x_{i}}\right)
$$

enthält selbst als Factor $V$, wie sich z. B. sofort zeigt, wenn man sie aufs Quadrat erhebt.

Die Gleichungen (14), (12) stellen alle Unterdeterminanten, welche aus den Gliedern der beiden vorletaten Reihen von (1) gebildet werden können, vollstündig von den Differentialen und unwesentlichen Factoren (kx) befreit vor. In der Determinantengleichung (1) Fommen ïbrigens nur die einfacheron Verbindungen, welche durch (14) ausgedrïcht sind, vor. Die Determinante (1) stellt demnach einen Complex rom Grade

$$
2[6(m+n+p)-19]
$$

vor, welcher die windschiefe Fläche in

$$
4 m n p(6(m+n+p)-19)
$$

Erzeugenden trifft. Nach unseren frïheren Erörterungen ist die Hälfte dieser Zahl der Grad der Curve der vierpuntigen Berïhrung.

Man erhälț dagegen die Zahl der vierpunktig berührenden Tangenten, welche eine willkührliche Gerade $z$ schneiden, wenn man in der Determinante (1) die letzte Horizontal- und Verticalreihe durch $z f z \varphi z \psi z x$ ersetzt. Daraus entnehmen wir den Satz:

Der Grad der von den vierpunktigen Tangenten gebildeten Linienfläche ist gleich

$$
4 m n p(6(m+n+p)-19) \text {. }
$$

Dieselbe hat mit der ursprünglichen Fläche die Curve der vierpunktigen Berührung gemein, welche als Durchschnittscurve vierfach zu zählen ist. Beide Flächen schneiden sich im Allgemeinen in einer zweiten Curve. 
Ersetzt man in der Determinante (1) die letzte Horizontal- und Verticalreihe durch $z f z \varphi z \psi z x$, so stellt dieselbe nach II. zugleich das Product der Gleichungen $y^{\prime} z=0, y^{\prime \prime} z=0$ der beiden speciellen linearen Complexe vor, deren Axen die beiden vierpunktigen Tangenten $y^{\prime}, y^{\prime \prime}$ sind. Diese Linien werden daher coincidiren, wenn der Kern der Determinante (1) verschwindet. Derselbe stellt einen Complex rom Grade

$$
4[3(m+n+p)-10]
$$

vor. Die Curve vierpunktiger Berïhrung besitzt also

$$
8 m n p[3(m+n+p)-10]
$$

Punlte, in denen sie von einer Erzeugenden der Fläche berithrt wird,

Es kann übrigens Linienflächen geben, für welche jede Erzengende zwei zusammenfallende vierpunktige Tangenten besitzt. Solche Flächen genügen einer partiellen Differentialgleichung, an deren vollstandiger Darstellung die Gleichungen (14) ausreichen. Dieselbe ist nämlich, wenn die Ausdrücke rechterhand in (14) mit $R_{1} R_{2}, R_{3}$, die Unterdeterminanten der Determinante $V$ mit $A_{i k}$ bezeichnet werden:

$$
\begin{gathered}
A_{11}=\mid \begin{array}{cc:c}
\varphi^{2} & \varphi \psi & \text { u. s.w. } \\
\varphi \psi & \psi^{2} & \text {. }
\end{array} \\
\Sigma R_{\imath} R_{k} A_{i k} \equiv 0 .
\end{gathered}
$$

Das Geschlecht der Curve vierpunktiger Berïhrung ist in diesem Falle gleich dem der Eläche.

Wir zeigen jetzt, wie sich mit Hülfe der gewonnenen Zahlen die Zahl der stationären Tangenten einer algebraischen Haupttangentencurve der Fläche vollständig bestimmen lässt.

Wenn nämlich die Fläche einem linearen Complexe $\Psi$ angehört, so kann man nach denjenigen Erzeugenden fragen, für welche eine der vierpunktigen Tangenten Gerade desselben ist. Je zwei Tangenten dieser Art sind aber conjugirte Polaren in Bezug auf den linearen Complex, sie coincidiren dahey in dem Falle, wo eine von ihnen zux Complexgeraden wird. In den $8 m n(3(n+n)-7)$ Berührungspunkten der Curve vierpunktiger Berührung mit den Erzeugenden der Fläche gehören demnach die vierpunktigen Tangenten dem linearen Complexe an. Aber diese viempuntitigen Tangenten sind dann zugleich Elemente der algebraischen Hauptangentencurve, welche durch den linearen Complex bestimmt ist, $d$. $h$. stationäre Tangenten derselben. Bezeichnet man daher durch $R, M, N, \Omega$ Rang, Ordnung, Classe, Zahl der stationären Tangenten der Curve, so gelten die folgenden Relationen: 


$$
\begin{aligned}
R & =12 m n(m+n-2) \\
M=N & =2 m n(m+n-1) \\
\Omega & =8 m n(3(m+n)-7)+4 m n(m+n-2) \\
& =9 m n(7(m+n)-16) .
\end{aligned}
$$

Die Haupttangentencurve schneidet dabei, wie sich sofort aus dem unter I. (1) entwickelten Satze ergiebt, jede Erzeugende in zwei Punkten, welche harmonisch liegen zu den beiden auf der letateren befindlichen Punkten der Curve vierpunktiger Berührung. Aus den von Zeuthen und Cayley entwickelten Formeln*):

$$
\begin{aligned}
& R=M(M-1)-2 h, \\
& M=R(R-1)-2 y-3 N-3 \Omega, \\
& N+\Omega=3(R-M), \\
& R=N(N-1)-2 g, \\
& N=R(R-1)-2 x-3 M-3 \Omega
\end{aligned}
$$

für die Singularitäten einer Raumcurve erhält man mit Benutzung der gegebenen Werthe die Zahlen $h, y, g, x$. Die dritte Formel ist übrigens eine Identität, eine Bemerkung, die als Controle der vorhergehenden Betrachtungen dienen kann. Insbesondere ist das Geschlecht der Haupttangentencurve

$$
\begin{aligned}
P & =2 m n[2(m+n)-5]+1 \\
& =4 P^{\prime}+2 m n .
\end{aligned}
$$

wo $P^{\prime}$ das Geschlecht der Fläche.

Wenn eine Fläche, für welche die partielle Differentialgleichung (15) besteht, einem linearen Complexe angehört, so sind alle vierpunktigen Tangenten Geraden des letzteren. Aber sie bilden zugleich stationäre Tangenten einer Haupttangentencurve. Die Curve vierpunktiger Berührung reducirt sich dann auf eine gerade Linie, während ausserdem noch eine krumme algebraische Haupttangentencurve vorhanden ist.

\section{VII.}

Ueber die vierpunktigen Tangenten specieller windschiefer Flächen.

Wenn die Fläche einem speciellen Complexe $F=\gamma x$ angehört, so besitzt sie eine $n p$-fache Leitlinie. Von den Cuspidalpunkten der Fläche liegen $2 n p(n+p-2)$ auf dieser Leitlinie, während die $.2 n p(n+p-2)$ anderen der krummen Doppelcurve angehören, welche

*) Vgl. Bulletin des Sc. Math. 1870, p. 150; Cremona, Theorie der OberAlächen S. $10 \mathrm{ff}$. 
die Fläche besitzt. Den Grad der letzteren erhält man, wenn man von dem Totalgrad der Doppelcurven

$$
n p[2 n p-(n+p)]
$$

die für $\frac{n p(n p-1)}{2}$ Doppelgerade zählende Leitlinie abzieht. Der Grad der eigentlichen Doppelcurve ist daher

$$
\frac{n p}{2}[3 n p-2(n+p)+1] \text {. }
$$

Da in einer beliebigen, durch die Leitlinie $\gamma$ gelegten Ebene nur $\frac{n p}{2}(n p-1)$ nicht auf der ersteren gelegene Punkte dieser Curve vorhanden sind, so erhält man als Zahl der Punlte, welche die Doppelcurve mit der Leitlinie gemein hat,

$$
n p[n p-(n+p)+1] \text {. }
$$

Die Leitlinie zählt zugleich $n p$-fach als Curve vierpunktiger Berïhrung. Den Beweis dafür kann man aus dem Umstande entnehmen, dass die Determinante VI. (I), für $f=y x,\left(\gamma^{2}\right)=0$ den Factor $a_{x} b_{\gamma}-b_{x} a_{y}=-p$ absondert, welcher sich auf die Leitlinie bezieht. Hiernach ergiebt sich als Grad der eigenthchen Carve vierpunltiger Bewizhrung

$$
3 n p[4(n+p)-9] \text {. }
$$

Wir bestimmen endlich noch die Zahl der Punkte, in denen diese Curve der Leitlinie begegnet. Da in jeder durch die Leitlinie gelegten Ebene $n p$ Erzeugenden also auch $n p$ Punkte der Curve liegen, die nicht auf der Axe gelegen sind, so muss die Curve mit der letzteren $4 n p(3(p+n)-7)$ Punkte gemein haben. Man überzeugt sich davon auch auf folgende Weise. Die Punkte, in denen die Curve der Leitlinie begegnet, sind offenbar auf solchen Erzeugepden gelegen, für welche die beiden vierpunktigen Tangenten coincidiren. Die Bedingung dieser Coincidenz ist aber das Verschwinden des Kerns der Determinante (1), welcher nach einfacher Redaction die Form

$$
\left|\begin{array}{ccc}
\gamma \varphi & \gamma \psi & 0 \\
(m-1) U_{2} & (p-1) U_{3} & V \\
\varphi d^{3} x & \psi d^{3} x & x d^{3} x
\end{array}\right|=0
$$

annimmt. Dieser Ausdruck repräsentirt einen Complex vom Grade $6(n+p)-14$, welcher mit den drei erzengenden Complexen $4 n p(3(n+p)-7)$ Erzeugende der Fläche bestimmt, für welche ein Punkt der eigentlichen Curve vierpunktiger Berïhrung auf der Leitlinie liegt.

Wir betrachten jetzt den Fall, wo unter den erzeugenden Complexen sich zwei specielle lineare $\alpha_{x} \beta_{x}$ befinden, deren Axen sich nicht schneiden. Alsdann kann offenbar ausser den beiden $p$-fach zählenden Leitlinien $\alpha, \beta$, welche die Fläche besitst, keine eigentliche Curve vier- 
punktiger Berührung vorhanden sein. Dies ergiebt sich auch aus der Betrachtung der Determinante VI. (1), welche sich auf

$$
\left|\begin{array}{ll}
\psi d^{2} x \psi d^{3} x \\
x d^{2} x x d^{3} x
\end{array}\right|^{2}(\alpha \beta)\left(a_{x} b_{c}-b_{x} a_{\alpha}\right)\left(a_{x} b_{\beta}-b_{x} a_{\beta}\right)=0
$$

reducirt, weil $U_{1}, U_{2}, f d^{3} x, \varphi d^{3} x$ sämmtlich verschwinden. Die beiden Factoren

$$
\begin{aligned}
& a_{x} b_{a}-b_{x} a_{c}=0 \\
& a_{x} b_{\beta}-b_{x} a_{\beta}=0
\end{aligned}
$$

beziehen sich auf die beiden Leitlinien, welche als Curven vierpunktiger Berührung ebenfalls $p$-fach zählen.

Der Factor

$$
\left|\begin{array}{ll}
\psi d^{2} x & \psi d^{3} x \\
x d^{2} x & x d^{3} x
\end{array}\right|
$$

gleich Null gesetzt, führt dagegen auf eine sehr merkwürdige Singularität dieser Classe von windschiefen Flächen. Da er nämlich die willkührlichen Grössen $b$, a gar nicht enthält, so liefert er eine Zahl besonderer Erzeugenden, welche unendlich viele vierpunktige Tangenten besitzen, deren drei consecutive Frzeugende also so gelegen sind, wie die Erzeugenden ein und derselben Art eines Hyperboloides. Solche Erzeugende mögen in Exmangelung einer passenderen Benennung als hyperbolische Erzeugende bezeichnet werden. Die Gleichungen (14) liefern dann die vollständige Darstellung dieses Factors in der Form:

$$
\left(\alpha \beta \psi x \frac{\partial V}{\partial x} \quad \Theta^{3}+\frac{\partial U_{3}}{\partial x}-\frac{\Omega^{3}}{V}\right)=0 .
$$

Derselbe stellt einen Complex rom Grade $2(3 p-4)$ vor. Die Anzahl der hyperbolischen Erzeugenden der Linienflüche mit zwei discreten windschiefen $p$-fachen Leitlinien ist daher

$$
4 p(3 p-4) \text {. }
$$

Die Singularitäten der algebraischen Haupttangentencurven der Fläche ergeben sich ohne weiteres aus den am Schlusse von § VI. angegebenen Formeln, wenn in denselben etwa $n=p, m=1$ gesetzt wirl. Jede Haupttangentencurve hat zwei stationäre Tangenten in den beiden (zu den Leitlinien harmonisch liegenden) Punkten, in denen sie einer hyperbolischen Erzeugenden begegnet.

Wir haben endlich noch den Fall zu beriücksichtigen, dass die windschiefe Fläche einem speciellen linearen $\alpha_{x}$ und dem linearen Complexe $\beta_{x}$ angehört, während $(\beta \alpha)=0$ ist. Der Complex, welcher die hyperbolischen Erzeugenden bestimmt, enthält dann den unwesentlichen Factor $(\omega \psi)$. Die Zahl der hyperbolischen Erzeugenden ist daher

$$
2 p(5 p-7) \text {. }
$$


Da ferner jede der algebraischen Haupttangenten den Erzeugenden nur in einem Punkte begegnet, so hat man für die Singularitäten derselben die folgenden Formeln:

$$
\begin{gathered}
R=6 p(p-1), \\
M=N=p(2 p-1), \\
\Omega=2 p(5 p-7), \\
R-2 P=2(M-1), \quad P=p^{2}-2 p+1 .
\end{gathered}
$$

Das Geschlecht der Haupttangentencurve muss mit dem Geschlechte der windschiefen Fläche identisch sein. In der That erhält man aus \& III. :

$$
P^{\prime}=p^{2}-2 p+1=P \text {. }
$$

Diese Curven besitzen daher $\frac{1}{2} p(p-1)\left(4 p^{2}-7\right)$ scheinbare Doppelpunkte u. s. w.

Man kann übrigens die Gleichung für die hyperbolischen Erzeugenden auch direct aufstellen. Damit überhaupt vier consecutive Erzeugende einem Hyperboloid, d. h. drei linearen Complexen angehören, müssen die Gleichungen

$$
\begin{aligned}
& c x=0, \\
& c d x=0, \\
& c d^{2} x=0 \\
& c d^{3} x=0
\end{aligned}
$$

drei wesentlich verschiedene Lösungen für die Coefficienten $c$ zulassen. Dazu aber ist erforderlich das Bestehen der 6 Gleichungen

$$
\mu_{1} x_{i}+\mu_{2} d x_{i}+\mu_{3} d^{2} x_{i}+\mu_{4} d^{3} x_{i}=0
$$

mit vier homogenen Unbekannten $\mu$. Es müssen also zwischen den $x$ drei wesentlich verschiedene Identitäten bestehen. Wenn nun die Fläche den Complexen $\alpha_{x} \beta_{x}$ angehört, so sind zwei derselben durch $\alpha_{x}=0, \beta_{x}=0$ selbst vertreten. Multiplicirt man endlich die sechs Gleichungen mit $\psi_{i}$ und summirt jedesmal wach $i$, so ergiebt sich

$$
\mu_{3} \psi d^{2} x+\mu_{4} \psi d^{3} x=0 \text {. }
$$

Dagegen durch Multiplication mit $x_{i}$

$$
\mu_{3} x d^{2} x+\mu_{4} x d^{3} x=0 .
$$

Die einzige erforderliche dritte Identität ist daher das Verschwinden der Determinante der letzteren beiden Gleichungen, welche mit dem obigen Ausdrucke identisch ist. Zugleich ist ersichtlich, dass diese Art von Erzeugenden im Allgemeinen nur bei den Flächen, welche 2wei linearen Complexen angehören, auftritt. Für eine beliebige windschiefe Fläche, welche durch den Sclnitt von $F \Phi \Psi$ gebildet wird, würde das Verschwinden der sämmtlichen Partialdeterminanten des Systemes 


$$
\left|\begin{array}{llll}
f d^{2} x & \varphi d^{2} x & \psi d^{2} x & x d^{2} x \\
f d^{3} x & \varphi d^{3} x & \psi d^{3} x & x \\
d^{3} x
\end{array}\right|
$$

erforderlich sein. Die äquivalenten Ausdrücke für diese Unterdeterminanten sind in $\S$ VI. vollständig gegeben.

\section{VIII.}

Deber ein Problem aus der Theorie der Ranmcurven.

Die Einführung und Elimination höherer homogener Differentiale, wie wir sie im $\S \nabla I$. durchgeführt haben, hat man bisher, wo die letzteren den zweiten Grad überstiegen, meist zu vermeiden gesucht und an Stelle derselben andere Differentiale eingeftihrt, welche nur vom ersten oder zweiten Grade sind. In der letzteren Weise ist z. B. von Clebsch ein Problem behandelt worden ${ }^{*}$, welches mit dem in \$ VI. entwickelten eine gewisse Verwandtschaft besitzt, die Bestimmung der Gleichung derjenigen Fläche, welche die vollständige Schnittcurve zweier Flächen $F=0, \Phi=0$ rom $m^{\text {ten }}$ und $n^{\text {ten }}$ Grade in den Wendungspunkten dieser Curve trifft. Aber dieser Weg führt bei unserem liniengeometrischen Problem zu keinem Resultate, weil die Curve vierpunktiger Berührung nicht eindeutig auf die Erzeugenden der Fläche bezogen werden kann. Es scheint daher nöthig, die höheren Differentiale beizubehalten. Man kann nun auch die f'rage nach den Wendungspunkten einer Raumeurve in einer ganz ähnlichen Weise behandeln, a. h. sich die Aufgabe stellen, aus der Determinante

$$
\Delta=\left(\begin{array}{llll}
x & d x & d^{2} x & d^{3} x
\end{array}\right)=0,
$$

welche fiur einen Inflexionspunkt verschwindet, die Differentiale $z u$ eliminiren. Das Resultat ist dann die Gleichung der gesuchten Fläche. Es mag gestattet sein, den Verlauf dieses Eliminationsprocesses zur Veranschaulichung der Rechnungen in $\S$ VI. hier noch auszuführen.

Zunächst gelten die Gleichungen:

$$
\left\{\begin{array}{c}
F=0, \quad \Phi=0, \\
f_{i} d x_{i}=0, \quad \varphi_{i} d x_{i}=0, \\
f_{i} d^{2} x_{i}+(m-1) f_{i k} d x_{i} d x_{k}=0, \quad \varphi_{i} d^{2} x_{i}+(n-1) \varphi_{i k} d x_{i} d x_{k}=0 \\
k_{i} d x_{i}=0, \quad k_{i} d^{2} x_{i}=0, \quad k_{i} d^{3} x_{i}=0
\end{array}\right.
$$

Wir setzen ferner

$$
\left\{\begin{array}{l}
(d x f \varphi b)=\left(d x^{2}\right)=\Delta^{\prime \prime}, \\
(f \varphi x d x)=\Delta^{\prime}
\end{array}\right.
$$

Dabei gilt dann die Beziehung

$$
\Delta^{\prime} \Delta^{\prime \prime}=-\left(d x^{2}\right)(k x)\left|\begin{array}{ll}
f^{2} & f \varphi \\
f \varphi & \varphi^{2}
\end{array}\right| .
$$

*) Crelle's Journal Bd. 63, S. 1 . 
Setzt man daher

so ist

$$
V=\left|\begin{array}{ll}
f^{2} & f \varphi \\
f \varphi & \varphi^{2}
\end{array}\right|
$$

Ausserdem hat man

$$
\Delta^{\prime}=-(k x) P \text {. }
$$

(4) $\quad f_{i k} d x_{i} d x_{k}=-(k x)^{2} U_{1}, \quad \varphi_{i k} d x_{i} d x_{k}=-\left(k_{k} x\right)^{2} U_{2}$,

wo $U_{1} U_{2}$ die Hess e'schen Determinanten von $f, \varphi$, gerändert respective mit $\varphi_{i} f_{i}$, bedeuten.

Die Gleichung der gesuchten Fläche nimmt eine sehr einfache Gestalt an, wenn man $\Delta$ mit $\Delta^{\prime \prime}$ multiplicirt. Es ergiebt sich

$$
\Delta \Delta^{\prime \prime}=(i x)\left(d x^{2}\right) F=0 \text {, }
$$

wo

$$
F=(f \partial x)\left(\varphi d^{2} x\right)-\left(f d^{2} x\right)\left(\varphi d^{3} x\right)=0 \text {. }
$$

Aus dem Ausdrucke $F$ sind nun noch die dritten Differentiale zu entfernen, da die zweiten nach (4) unmittelbar ersetzt werden können. Dazu verfährt man falgendermassen. Durch Differentiation von (2) entsteht:

$$
\left\{\begin{array}{l}
f_{i} d^{3} x_{i}+(m-1) f_{i k} d x_{k} d^{2} x_{i}=(m-1)(k x)^{2} \frac{\partial U_{1}}{\partial x_{i}} d x_{i} \\
\varphi_{i} d^{3} x_{i}+(n-1) \varphi_{i k} d x_{k} d^{2} x_{i}=(n-1)(k x)^{2} \frac{\partial U_{2}}{\partial x_{i}} d x_{i}
\end{array}\right.
$$

Um aus diesen Gleichungen die zweitep Differentiale zu entfernen, setzen wir:

$$
\left\{\begin{array}{l}
f_{i i} d x_{k} d^{2} x_{i}=\Pi_{1}, \\
f_{i} d^{2} x_{2}=(m-1)(k x)^{2} U_{1}, \\
\varphi_{i} d^{2} x_{i}=(n-1)\left(k_{x}\right)^{2} U_{2}, \\
k_{i} d^{2} x_{i}=0, \\
\varphi_{i k} d x_{k} d^{2} x_{i}=\Pi_{2} .
\end{array}\right.
$$

Hieraus entsteht durch Elimination der $d^{2} x_{i}$ :

(9) $\Pi_{1} A_{1}-(m-1)(k x)^{2} V_{1} \Omega_{4}+(n-1)(k x)^{2} U_{2} \Omega_{2}+\Pi_{2} A_{2}=0$.

Dabei ist

so dass:

$$
\begin{aligned}
& A_{2}=\left(f_{i k} d x_{k}, f_{i}, \varphi_{i}, k_{i}\right)=-(k x)^{2} U_{1}, \\
& A_{1}=(k x)^{2} U_{2}
\end{aligned}
$$

$$
\Pi_{1} U_{2}-\Pi_{2} U_{1}=(m-1) U_{1} \Omega_{1}-(n-1) U_{2} \Omega_{2} \text {. }
$$

Aber aus (7) hat man

$$
\text { (11) } \begin{aligned}
\frac{F}{(m-1 n-1} & =(k x)^{2}\left(U_{2} \frac{\partial U_{i}}{\partial x_{i}} d x_{i}-U_{1} \frac{\partial U_{2}}{\partial x_{i}} d x_{i}\right)-\left(U_{2} \Pi_{1}-\Pi_{2} U_{1}\right) \\
& =(k x)^{2}\left(U_{2} \frac{\partial U_{1}}{\partial x_{i}} d x_{i}-U_{1} \frac{\partial U_{2}}{\partial x_{i}} d x_{i}\right)-\left((m-1) U_{1} \Omega_{i}-(n-1) U_{2} \Omega_{2}\right) .
\end{aligned}
$$


A. Voss.

In den Ausdrücken $\Omega_{1} \Omega_{2}$ lassen sich nun die ersten Differentiale, die in ihnen noch enthalten sind, durch die ihnen proportionalen Unterdeterminanten ersetzen. Man hat

$$
\begin{aligned}
& \Omega_{1}=-\sum_{k}\left|\begin{array}{cccc}
\varphi_{1 k} & \varphi_{2 k} & \varphi_{3 k} & \varphi_{4 k} \\
\varphi_{1} & \varphi_{2} & \varphi_{3} & \varphi_{4} \\
k_{1} & k_{2} & k_{3} & k_{4} \\
f_{1 k} d x_{k} & f_{2 k} d x_{k} & f_{3 k} d x_{k} & f_{4 k} d x_{k}
\end{array}\right| d x_{k} \\
& \varphi_{11} \varphi_{12} \varphi_{13} \varphi_{14} f_{1} \varphi_{1} k_{1} \text {, } \\
& \begin{array}{lllllll}
\varphi_{21} & \varphi_{22} & \varphi_{23} & \varphi_{24} & f_{2} & \varphi_{2}{ }^{*} k_{2}
\end{array} \\
& \begin{array}{lllllll}
\varphi_{31} & \varphi_{32} & \varphi_{33} & \varphi_{34} & f_{3} & \varphi_{3} & z_{3}
\end{array} \\
& =-\Sigma \mid \begin{array}{llllllll}
\varphi_{41} & \varphi_{42} & \varphi_{43} & \varphi_{44} & f_{4} & \varphi_{4} & k_{4} & d x_{k}
\end{array} \\
& \begin{array}{lllllll}
\varphi_{1} & \varphi_{2} & \varphi_{3} & \varphi_{4} & 0 & 0 & 0
\end{array} \\
& \begin{array}{lllllll}
k_{1} & k_{2} & k_{3} & k_{4} & 0 & 0 & 0
\end{array} \\
& \begin{array}{llllllll}
f_{1 k} & f_{2 k} & f_{3 k} & f_{4 k} & 0 & 0 & 0
\end{array} \\
& \begin{array}{lllll}
\varphi_{11} & \varphi_{12} & \varphi_{13} & \varphi_{14} & f_{1}
\end{array} \\
& \begin{array}{lllll}
\varphi_{21} & \varphi_{22} & \varphi_{23} & \varphi_{24} & f_{2}
\end{array} \\
& =(k x)^{2} \Sigma \mid \varphi_{31} \varphi_{32} \varphi_{33} \varphi_{34} f_{3} d x_{k} . \\
& \varphi_{41} \varphi_{42} \varphi_{43} \varphi_{44} f_{4} \\
& \begin{array}{lllll}
f_{1 k} & f_{2 k} & f_{3 k} & f_{1 k} & 0
\end{array}
\end{aligned}
$$

Setzt man daher:

so ist

$$
\begin{aligned}
& \Omega_{1}=(k x)^{2} \Theta_{i}^{\prime} d x_{i}, \\
& \Omega_{2}=(k x)^{2} \Theta_{i}^{\prime \prime} d x_{i},
\end{aligned}
$$

Daher erhält man:

$$
\Theta_{i}^{\prime} x_{i}=U_{2}, \quad \Theta_{i}^{\prime \prime} x_{i}=U_{1}
$$

$$
\text { (12) } \begin{aligned}
& \frac{-F}{(k x)^{2}(m-1)(n-1)}= \mid \begin{array}{l}
U_{1}\left(\frac{\partial U_{1}}{\partial x_{i}}+(n-1) \Theta_{i}^{\prime \prime}\right) d x_{i} \\
U_{2}\left(\frac{\partial U_{2}}{\partial x_{i}}+(m-1) \Theta_{i}^{\prime}\right) d x_{i}
\end{array} \\
&=\frac{1}{3(m+n-3)}\left(\begin{array}{l}
\left(\frac{\partial U_{1}}{\partial x_{i}}+(n-1) \Theta_{i}^{\prime \prime}\right) x_{i}\left(\frac{\partial U_{1}}{\partial x_{i}}+n-1 \Theta_{i}^{\prime \prime}\right) d x_{i} \\
\left(\frac{\partial U_{2}}{\partial x_{i}}+(m-1) \Theta_{i}^{\prime}\right) x_{i}\left(\frac{\partial U_{2}}{\partial x_{i}}+m-1 \Theta_{i}^{\prime}\right) d x_{i}
\end{array}\right.
\end{aligned}
$$

Aus der rechten Seite entfernt man endlich die Differentiale vermöge der Identität

$$
\Delta^{\prime}\left(f_{i} \varphi_{i} c_{i} b_{i}\right)=V\left|\begin{array}{ll}
c_{x} & c_{d x} \\
b_{x} & b_{d x}
\end{array}\right|
$$

welche für die willkührlichen Grössen $c_{i} b_{i}$ die Gleichung:

$$
\left|\begin{array}{ll}
c_{x} & c_{d x} \\
b_{x} & b_{d x}
\end{array}\right|=-(k x)\left(f_{i} \varphi_{i} c_{i} b_{i}\right)
$$


liefert. Demnach ist

$$
F \equiv\left(f_{i}, \varphi_{i}, \quad \frac{\partial U_{1}}{\partial x_{i}}+(n-1) \Theta_{i}^{\prime \prime}, \quad \frac{\partial U_{2}}{\partial x_{i}}+(m-1) \Theta_{i}^{\prime}\right)=0
$$

die Gleichung der gesuchten Fläche und zwar in der bereits von Clebsch gegebenen Form.

IX.

Die Punkte fünfpunktiger Berührung auf der windschiefen Iläche.

Soll eine Gerade $y$ fünf consecutive Erzengende schreiden, so muss $\left(y^{2}\right)=0, \quad(y x)=0, \quad(y d x)=0, \quad\left(y d^{2} x\right)=0, \quad\left(y d^{3} x\right)=0, \quad\left(y d^{4} x\right)=0$ sein. Die Elimination der $y$ liefert sofort die Deterninante

$$
\left(\Omega: x d x \quad d^{2} x d^{3} x d^{4} x\right)=0 .
$$

Sie geht durch Multiplication mit $\Delta^{2}$ in die Determinante VI. (1) über, wenn man die letzte Horizontal- und Vertikalreihe derselben durch:

$$
f d^{4} x \varphi d^{4} x \psi d^{4} x \quad x d^{4} x \quad 000
$$

ersetzt. Berücksichtigt man die Gleichangen VI. (12), (14), so ist ersichtlich, dass an Stelle der vierten Differentiale noch ihre äquivalenten Ausdrücke einzufïhren sind. Dabei müsste sich der Factor $(k x)^{8}$ absondern lassen, vorausgesetzt, dass die Punkte fünfpunktiger Berührung überhaupt auf Erzeugenden liegen, welche vollständiger Schnitt ron vier Complexen sind. In diesem Falle würde die Zahl der fraglichen Punkte

$$
20 m n p(2(m+n+p)-7)
$$

sein. Die Reduction der Determinante, selbst in dem einfacheren Falle, wo der eine der Complexe linear ist, hat mir aber nicht gelingen wollen. Wahrscheinlich ist ubrigens, dass analog wie in der allgemeinen Flächentheorie, die fünfpunktigen Tangenten sich nicht durch den vollständigen Schnitt von vier Complexen darstellen lassen. Es pag noch bemerkt werden, dass im Allgemeinen die Linienflächen, welche unendlich vieler linearen Complexen angehören, keine fünfpunktigen Tangenten besitzen. Ihr Auftreten würde die Existenz von Erzeugenden bedingen, deren vier unmittelbar folgende mit ihnen demselben Hyperboloid angehören, also eine weit höhere Singularität vorstellen.

X.

Das osculirende Hyperboloid der windschiefen Flächen.

Die Erzeugenden des osculirenden Hyperboloides, welches zu der Erzeugenden $x$ der Fläche gehört, sind die Haupttangenten $y$ der letzteren, welche den Punkten von $x$ entsprechen. Man hat daher für die Erzeagenden des Hyperboloides die Gleichungen V. (1): 


$$
\left(y^{2}\right)=0, \quad(y x)=0, \quad(y d x)=0, \quad\left(y d^{2} x\right)=0 .
$$

Wir stellen die Gleichung dieses Hyperboloides in Liniencoordinaten dar. Damit eine Gerade z zwei unendlich nahe Erzengende $y$ schneide, muss das System der Gleichungen (1) mit

$$
(z y)=0
$$

eine Doppelwurzel besitzen. Es muss also die Determinante

$$
\left(\Omega: x d x d^{2} x z\right)
$$

verschwinden. Durch Multiplication mit $\Delta^{2}$ geht dieselbe über in:

$$
\begin{array}{cccccc}
f^{2} & f \varphi & f \psi & 0 & (m-1) U_{1} & f z \\
f \varphi & \varphi^{2} & \varphi \psi & 0 & (n-1) U_{2} & \varphi z \\
f \psi & \varphi \psi & \psi^{2} & 0 & (p-1) U_{3} & \psi z \\
0 & 0 & 0 & 0 & -V & x z \\
(m-1) U_{1} & (n-1) U_{2} & (p-1) U_{3} & V & 0 & 0 \\
f z & \varphi z & \psi z & x z & 0 & 0
\end{array}
$$

Die Gleichung (4) ist die Gleichung des osculirenden Hyperboloides in Liniencoordinaten. Sind die drei Complexe $F \Phi \psi$ linear, so reducirt sie sich, da $U_{1} U_{2} U_{3}$ verschwinden, auf die Complexgleichung des von denselben erzengten Hyperboloides, multiplicirt mit dem Factor $\left.V^{2}{ }^{*}\right)$. Befinden sich unter den Complexen zwei lineare specielle $\alpha_{x}, \beta_{x}$, so reducirt sich für $(z \alpha)=0,(z \beta)=0$ die Determinante (4) auf:

$$
\left|\begin{array}{cc}
\psi z & p-1 U_{3} \\
x z & V
\end{array}\right|=0
$$

Für eine singuläre Erzeugende der Fläche reducirt sich (4) auf $(x z)^{2} K, d$. h. auf den doppeltzählenden speciellen linearen Complex mit der Axe $x$. Das osculirende Hyperboloid wird endlich unbestimmt, wenn der Factor $K$ verschwindet, d. h. wenn:

$$
\left|\begin{array}{cccc}
f^{2} & f \varphi & f \psi & (m-1) U_{1} \\
f \varphi & \varphi^{2} & \varphi \psi & (n-1) U_{2} \\
f \psi & \varphi \psi & \psi^{2} & (p-1) U_{3} \\
(m-1) U_{1} & (n-1) U_{2} & (p-1) U_{3} & 0
\end{array}\right|=0
$$

eine Gleichung, welche aussagt, dass zwei singuläre Erzengende znsammengerückt sind.

Die Tangenten des osculirenden Hyperboloides, welche die Erzeugende $x$ schneiden, sind gegeben durch die Gleichungen: 


$$
(x z)=0\left|\begin{array}{cccc}
f^{2} & f \varphi & f \psi & f z \\
f \varphi & \varphi^{2} & \varphi \psi & \varphi z \\
f \psi & \varphi \psi & \psi^{2} & \psi z \\
f z & \varphi z & \psi z & 0
\end{array}\right|==0 .
$$

Der zweite Ausdruck ist für $(x z)=0$ das Quadrat einer in den $z$ linearen Function, nämlich

$$
\frac{1}{(k x)}(f \varphi \psi x k z)
$$

Er repräsentirt einen linearen Complex, dessen den Punkten von $x$ zugehôrige Ebenen zugleich Tangentenebenen der Fläche sind. Wenn dagegen $(x z)$ nicht verschwindet, so stellt die zweite Gleichung (6) ein Hyperboloid vor, welches jeder Erzengenden ebenfalls zugeordnet ist, aber von dem osculirenden resschieden ist. Während das osculirende Hyperboloid die Erzeugenden $x, x+d x, x+2 d x+d^{2} x$ zu Erzeugenden zweiter Art enthält, hat das Hyperboloid (6) im Allgemeinen nur $x, x+d x$ za solchen. Die Erzeugende $x+2 d x+d^{2} x$ ist nicht einmal eine der Tangenten der letzteren, dies wird vielmehr nur für besondere Erzeugende der Fläche stattfinden, für welche die Gleichung (5) besteht, deren Anzahl mithin

ist.

$$
\text { 4. } m n p(3(m+n+p)-10)
$$

Ebenso gehört zu den Tangenten des osculirenden Hyperboloides im Allgemeinen nicht die Erzeugende $x+3 d x+3 d^{2} x+d^{3} x$. Es findet dies, wie eine einfache Betrachtung zeigt, nur statt für diejenigen Erzeugenden, welche von der Curye vierpunktiger Berülaung berührt werden. Aus der für den Fall zweier linearer Complexe gültigen Formel $\left(4^{\mathrm{a}}\right)$ erhält man insbesondere, wenn anstatt $z d^{3} x$ gesetzt wird, wieder die Bedingsgleichung für die hyperbolischen Erzeugenden, wie zu erwarten war.

\section{XI.}

\section{Ueber windschiefe Flächen vom Geschlechte Null.}

Wir gehen jetzt zu einem zweiten Theile von Untersuchungen über, die sich auf windschiefe Flächen beziehen, welcher mit dem. - vorigen nur insofern zusammenhängt, als dabei die pämlichen Methoden der Betrachtung zu Grunde gelegt werden. Die windschiefen Flächen rom Geschlechte Null gestatten in der That eine wesentlich andere Behandlung. Man kann eine solche Fläche auffassen als Inbegriff der Verbindungsgeraden sich eindeutig entsprechender Punkte zweier ihrer ebenen Schnittcurven*). Die Erzeugenden sind alsdann rationale

-) Indem man die windschiefen Flächen beliebigen Geschlechtes von diesem Gesichtspunkte aus betrachtet, gelangt man zu einer wesentlich anderen linien- 
Functionen eines Parameters $\lambda$, welche man erhält, wenn man die Ausdriúcke $p_{i k}$ aus den Coordinaten entsprechender Punkte der beiden Curven bildet und ron ibnen zu den allgemeinen Linieucoordinatell $x$ übergeht.

Wir können daher die Gleichung der Fläche in der Form

$$
\varrho x_{i}=\varphi_{i} \lambda
$$

schreiben. Es bedeuten hier die $\varphi_{i}$ rationale Functionen $n^{\text {ten }}$ Grades von $\lambda$, zwisehen denen die Identität

$$
\left(\varphi^{2}\right)=0
$$

vermöge der Coefficienten, welche in $\varphi_{i}$ auftreten, besteht. In Folge dessen kann man durch Differentiation nach $\lambda$ aus (2) eine weitere Reihe von Identitäten

$$
\left(\varphi \frac{\partial \varphi}{\partial \lambda}\right)=0,\left(\frac{\partial \varphi}{\partial \lambda}\right)+\left(\varphi \frac{\partial^{2} \varphi}{\partial \lambda^{2}}\right)^{2}=0 \text { u. s. w. }
$$

ableiten. Es empfiehlt sich, die Gleichungen (1) durch Einführung eines zweiten Parameters $\mu$ homogen zu machen. Es gelten dann die bekannten Identitäten:

sowie auch

$$
n \varphi_{i}=\lambda \frac{\partial \varphi_{i}}{\partial \lambda}+\mu \frac{\partial \varphi_{i}}{\partial \underline{\mu}} \text { u. s. w. }
$$

$\left(\varphi \frac{\partial \varphi}{\partial \mu}\right)=0,\left(\frac{\partial \varphi}{\partial \lambda} \frac{\partial \varphi}{\partial \mu}\right)+\left(\varphi \frac{\partial^{2} \varphi}{\partial \mu \partial \lambda}\right)=0, \quad\left(\frac{\partial \varphi}{\partial \mu}\right)^{2}+\left(\varphi \frac{\partial^{2} \varphi}{\partial \mu^{2}}\right)=0 \cdots$

Wir bestimmen nun zunächst die Singularitäten der durch die Gleichungen (1) dargestellten Fläche.

Die Ordnung der Fläche ist n. Denn die Bedingung, unter welcher die willkührliche Gerade $y$ eine Erzeugende $x$ derselben schneidet, ist:

$$
(y \varphi)=0
$$

eine Gleichung $n^{\text {tea }}$ Grades für $\lambda$.

Der Rang der Fläche ist $2(n-1)$. Eine Tangente $y$ der Fläche muss den Gleichungen

oder

$$
(y x)=0, \quad(y d x)=0,
$$

$$
\left(y \frac{\partial \varphi}{\partial \lambda}\right)=0, \quad\left(y \frac{\partial \varphi}{\partial \mu}\right)=0
$$

genügen. Die Elimination von $\lambda$ aus den beiden letzteren liefert eine Gleichung $2(n-1)^{\text {ten }}$ Grades in $y$, welche die Fläche in Liniencoordinaten darstellt. Die Fläche enthält daher eine Doppelcurvè vom Grade $\frac{(n-1)(n-2)}{2}$, wie übrigens auch aus dèm Geschlecht des ebenen Schnittes gefunden werden konnte.

geometrischen Darstellung derselben überhaupt, über die ich bei einer demnächstigen Gelegenheit handeln werde. 
Man kann übrigens den Grad der Doppelcurve anch direct bestimmen, wie wir jetzt zeigen wollen. Zwei Erzeugende $x, x^{\prime}$, denen die Parameter $\lambda, \lambda_{1}$ entsprechen, bilden einen Punkt der Doppeleurve, wenn oder

$$
\left(x x^{\prime}\right)=0
$$

$$
\left(\varphi_{1} \varphi\right)=0 \text {. }
$$

Da aber $\left(\varphi^{2}\right),\left(\varphi_{1}^{2}\right)$ identisch versehwinden, kann an Stelle der Gleichung $\left(\varphi \varphi_{1}\right)=0$ gesehrieben werden:

$$
\Sigma\left(\varphi_{i}-\varphi_{i}^{\prime}\right)^{2}=0 .
$$

In dieser Form enthält die Gleichung (4) den abzusondernden Factor $\left(\lambda-\lambda_{1}\right)^{2}$ und ist dann in Bezug auf $\lambda$ und $\lambda_{1}$ vom $n-2^{\text {ten }}$ Grade, In dieser reducirten Gestalt wollen wir sie durch

$$
\Psi\left(\lambda \lambda_{1}\right)=0,
$$

bezeichnen. Aus (5) ersehen wir zunächst, dass jede Erzeugende von $(n-2)$ anderen getroffen wird oder $n-2$ Punkte der Doppeleurve enthält. Ein willkührlicher Strahl des Büschels $x x^{\prime}$ ist ferner $x+\varrho x^{\prime}$. Damit derselbe zwei willhürliche Geraden $a, b$ schneide, muss:

$$
a_{x} b_{x^{\prime}}-b_{x} a_{x^{\prime}}=0
$$

sein. Die letztere Gleichung, durch $\lambda-\lambda_{1}$ theilbar, ist in Bezug auf $\lambda, \lambda_{1}$ vom Grade $n-1$. Eliminirt man jetzt aus $(5)$, $(6)$ etwa $\lambda_{1}$, so entsteht eine Gleichung rom $2(n-2)(n-1)^{\text {ten }}$ Grade in $\lambda$. Die Hälfte dieser Zahl stellt demnach solche Punlite der Doppelcurve vor, für welche eine in der Elbene $x x^{\prime}$ - der Doppelebene der Fläche durch den Punkt $x x^{\prime}$ gezogene Gerade die $a, b$ schneidet. Schneiden sich also $a$ und $b$, so liegt entweder ein Punkt der Doppeleurve in der Ebene $a b$, oder es geht eine Doppelebene durch den gleichnamigen Pankt. Da die Ordnung der Doppelcurve gleich der Classe der Doppeldeveloppabeln ist, so ist jede dieser Zahlen gleich $\frac{(n-1)(n-2}{2}$.

Man kann auch die Discriminante der Gleichung (5) bilden. Sie sagt aus, dass eine Erzeugende $x$ won zwei unendlich nahen Erzeugenden getroffen wira, oder dass eine Erzeugende von der Doppelcurve berithrt wird. Die Zahl dieser ausgeweichneten Erzeugenden ist daher:

$$
2(n-2)(n-3) \text {. }
$$

Wir bestimmen ferner die Zahl der dreifachen Tangentenebenen, sowie die ihr gleiche der dreifachen Punkte der Fläche. Eine Erzeugende mit dem Parameter $\lambda$ gehört $z \mathfrak{u}$ einer der beiden Singularitäten, wenn für irgend zwei Wurzeln

\section{der Gleichung (5) die Beziehung}

$$
\lambda_{i}^{\prime} \lambda_{k}^{\prime}
$$

$$
\Psi\left(\lambda_{i}^{\prime}, \lambda_{k}^{\prime}\right)=0
$$


besteht. Es muss also die symmetrische Function

$$
\nu=\Pi\left(\Psi\left(\lambda_{i}^{\prime} \lambda_{k}^{\prime}\right)\right)
$$

verschwinden, welche man durch Multiplication der $\frac{(n-2)(n-3)}{2}$ Polynome $\Psi\left(\lambda_{i}^{\prime} \lambda_{k}^{\prime}\right)$ bilden kann. Man übersieht leicht, dass, wenn man $V$ als rationale Function der Coefficienten von (5) darstellt, dabei $\lambda$ höchstens im Grade $(n-2)^{2}(n-3)$ auftreten wird. Diese Zahl, vermindert um die Zahl der Erzeugenden, welche die Doppelcurve berühren, giebt als Rest:

$$
(n-2)(n-3)(n-4) \text {. }
$$

Derselbe bezieht sich auf die beiden dualistischen Singularitäten und zwar wieder auf jede dreifach. Daher ist

$$
\frac{1}{6}(n-2)(n-3)(n-4)
$$

die Zahl der dreifachen Punkte und Ebenen der Fläche.

Die singulären Erzeugenden der Fläche sind durch

$$
\left(\frac{\partial \varphi^{2}}{\partial \lambda}\right)=0
$$

gegeben. Die letztere Gleichung ist in Folge der Identität $\left(\varphi^{2}\right)=0$ rom Grade $2(n-2)$. Man erhält sie in dieser reducirten Gestalt, wenn $\operatorname{man}$ in (5) $\lambda=\lambda_{1}$ setzt, also:

$$
\psi(\lambda \lambda)=0 \text {. }
$$

Die Zahl der singulären Erzeugenden ist daher $2(n-2)$.

Die bisher bestimmten Zahlen sind zunächst unter der Voraussetzung gewonnen, dass die Fläche keine besonderen Singularitäten, wie Doppel- oder Rückkehrerzeugende besitzt. Hine Doppelerzeugende wird auftreten, wenn die sechs Gleichungen

$$
\varphi_{i} \lambda=\varphi_{i} \lambda^{\prime}
$$

für verschiedene Werthe von $\lambda, \lambda^{\prime}$ erfüllt werden können. Das Auftreten einer Doppelerzeugenden modificirt übrigens weder den Rang der Fläche noch die Zahl der singulären Erzengenden, während die Doppelcurve sich für jede um zwei Einheiten erniedrigt.

Wenn aber die Gleichungen

$$
\frac{\partial \varphi_{i}}{\partial \lambda}=\varrho \varphi_{i}
$$

bestehen, so wird jede Gerade $y$, welche die Fläche in der Erzengenden $x$ schneidet, dieselbe in zwei zusammenfallenden Punkten treffen. Die Geraden $y$ aber, welche ausserdem noch dem linearen Complexe

$$
\left(y \frac{\partial^{2} \varphi}{\partial \lambda^{2}}\right)=0
$$

angehören, schneiden die Fläche in je drei zusammenfallenden Punkten. Eine solche Erzeugende ist daher Rückkehrgeneratrix der Fläche, und die Gleichung (10) giebt die Rückkehrtangentialebenen derselben längs 
der Erzeugenden $x$ durch die Ebenen des linearen Complexes an, welche den Punkten von $x$ zlugeordnet sind. Das Vorhandensein einer Rückkehrgeneratrix aber hat zur Folge, dass bei der Bestimmung der Gleichung der Fläche in Liniencoordinaten ein linearer specieller Complex sich aussondert. Bezeichnet man daber dureh $d$, die Zahl der Doppelund Rückkehrgeneratricen, durch $D$ den Grad der krummen Doppelcurve, so ist der Rang der Fläche gegeben durch die identischen Ausdrücke

$$
2(n-1)-r=n(n-1)-2 D-2 d-3 r .
$$

Für eine Rückkehrgeneratrix verschwindet ferner nicht allein

sondern auch

$$
\Psi(\lambda \lambda)=\left(\frac{\partial \varphi^{2}}{\partial \lambda}\right)=0,
$$

$$
\frac{\partial \psi \lambda \lambda}{\partial \lambda}=\left(\frac{\partial \varphi}{\partial \lambda} \frac{\partial^{2} \varphi}{\partial \lambda^{2}}\right)=\left(\varphi \frac{\partial^{2} \varphi}{\partial \lambda^{2}}\right)=\left(\frac{\partial \varphi}{\partial \lambda} \frac{\partial \varphi}{\partial \lambda}\right)=0 .
$$

Es hat also $\Psi \lambda \lambda=0$ für jede Rückkehrgeneratrix eine Doppelwurzel. $\Psi \lambda \lambda=0$ kann andere Doppelwurzeln haben, welche sich auf zusammengefallene singuläre Erzeugende beziehen. Die Zahl der singulären Erzeugenden ist daher:

$$
2(n-2)-2 r \text {. }
$$

Wir wenden uns jetzt zur Betrachtung der Haupttangenten der Fläche. . An Stelle der Gleichungen

$$
(y \varphi)=0, \quad\left(y \frac{\hat{\partial} \varphi}{\partial \hat{\lambda}}\right)=0, \quad\left(y \frac{\partial^{2} \varphi}{\partial \lambda^{2}}\right)=0,
$$

welche eine Haupttangente $y$ charakterisiren, setzen wir die äquivalenten Gleichungen:

$$
\left(y \frac{\partial^{2} \varphi}{\partial \lambda^{2}}\right)=0, \quad\left(y \frac{\partial^{2} \varphi}{\partial \lambda \partial \mu}\right)=0, \quad\left(y \frac{\partial^{2} \varphi}{\partial \mu^{2}}\right)=0 .
$$

Die Zahl der Haupttangenten, welche zwei willkührliche Gerade $a, b$ schneiden, ist daher bestimmt durch die Gleichung:

$$
\left(\Omega: \frac{\partial^{2} \varphi}{\partial \lambda^{2}} \frac{\hat{\partial}^{2} \varphi}{\partial \lambda \partial \mu} \frac{\partial^{2} \varphi}{\partial \mu^{2}} a b\right)=0
$$

Die windschiefe Fläche, welche von Haupttangenten gebildet wird, die einem linearen Complexe angehören, ist daher vom Grade

$$
6(n-2)
$$

Ordnung und Classe des Strahlensystems der Haupttangenten ist somit gleich $3(n-2)$, was mit der directen Abzählung übereinstimmt.

Die Linien vierpunktiger Berührung genügen den Bedingungen:

$$
\left(y \frac{\partial^{3} \varphi}{\partial x^{3}}\right)=0,\left(y \frac{\partial^{3} \varphi}{\partial \lambda^{2} \partial \mu}\right)=0,\left(y \frac{\partial^{3} \varphi}{\partial \partial \partial \mu^{2}}\right)=0,\left(y \frac{\partial^{3} \varphi}{\partial \mu^{3}}\right)=0,\left(y^{2}\right)=0,
$$

Fügt man denselber $(y z)=0$ hinzu, so ergiebt sich: 
Die Ordnung der von den vierpunlitigen Tangenten gebildeten windschiefen Fläche ist gleich $8(n-3)$. Die gleiche Zahl solcher Tangenten gehört ẗberhaupt einem willkährlichen linearen Complexe an. Wir schliessen dann weiter: Die Zahl der Punkte, in denen die Curve vierpunktiger Berïhrung eine Erzeugende berïhrt, ist gleich $8(n-3)$.

Je zwei Erzeugende der Fläche der vierpunktigen Tangenten ordnen sich rational den Erzeugenden der gegebenen Fläche zu. Die erstere Fläche ist daher von hyperelliptischem Charakter, ihre sämmtlichen Erzeugenden lassen sich als Functionen ron $\lambda$ darstellen, in denen eine quadratische Irrationalität auftritt. Es ist leicht, diese Functionen selbst anzugeben. $Z u$ diesem $Z$ wecke bezeichnen wir die Determinante

$$
\left(\frac{\partial^{3} \varphi}{\partial \lambda^{3}} \frac{\partial^{3} \varphi}{\partial \lambda^{2} \partial \mu} \frac{\partial^{3} \varphi}{\partial \lambda \partial \mu^{2}} \frac{\partial^{3} \varphi}{\partial \mu^{3}} y \varrho\right)
$$

in welcher die Grössen $\varrho$ willkührliche Coefficienten vorstellen, durch $\Omega$.

Man hat unter dieser Voraussetzung aus den Gleichungen (11)

$$
y_{i}=\mu \frac{\partial \Omega}{\partial \varrho_{i}}
$$

oder, wenn die Unterdeterminanten von $\Omega$ nach $y_{i} \varrho_{k}$ dureh $\Omega_{i k}$ bezeichnet werden:

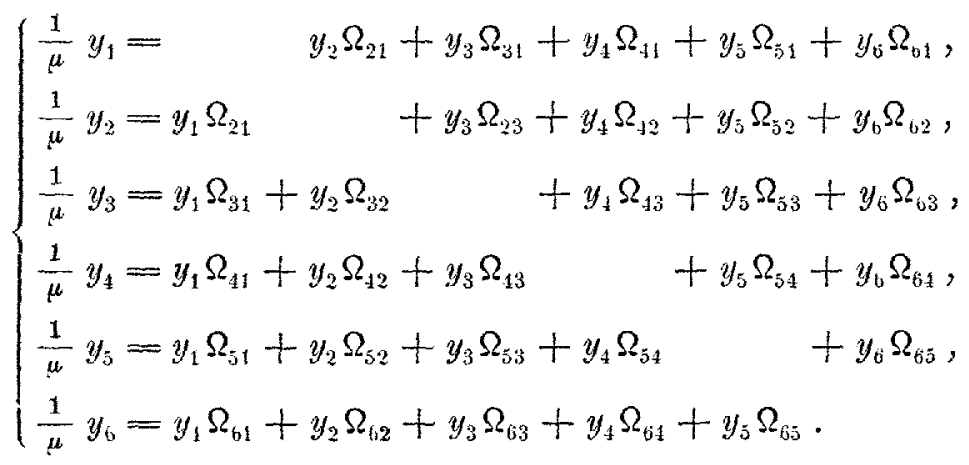

Die Gleichungen (12) geben, wenn $\mu$ bestimmt ist, die $y$ ausgedräckt durch rationale Functionen von $\lambda$ and $\mu$. Die Grösse $\mu$ bestimmt man auf folgende Weise. Man hat:

$$
\Omega^{2}=\left|\begin{array}{cccccc}
A_{11} & A_{12} & A_{13} & A_{14} & 0 & a_{1} \\
A_{21} & A_{22} & A_{23} & A_{24} & 0 & a_{2} \\
A_{31} & A_{32} & A_{33} & A_{34} & 0 & a_{3} \\
A_{41} & A_{42} & A_{43} & A_{44} & 0 & a_{4} \\
0 & 0 & 0 & 0 & 0 & \varrho y \\
a_{1} & a_{2} & a_{3} & a_{4} & \varrho y & \varrho^{3}
\end{array}\right|=-(\varrho y)^{2} \Delta^{\prime}
$$


Es bedeuten dabei die $A_{11} A_{12} \ldots$ die Ausdrüake

$$
{ }^{\prime} \Sigma\left(\frac{\partial^{3} \varphi_{i}}{\partial \lambda^{3}}\right)^{2} \quad \Sigma \frac{\partial^{3} \varphi_{i}}{\partial \lambda^{3}} \frac{\partial^{3} \varphi_{i}}{\partial \lambda^{2} \partial \mu} \ldots,
$$

$\Delta^{\prime}$ die Determinante:

$$
\begin{array}{llll}
A_{11} & A_{12} & A_{13} & A_{14} \\
A_{21} & A_{22} & A_{32} & A_{24} \\
A_{31} & A_{32} & A_{33} & A_{31} \\
A_{11} & A_{42} & A_{43} & A_{41}
\end{array} \mid,
$$

welche, gleich Null gesetzt, die Berührungspunkte der Curve vierpunktiger Berührung mit den Erzeugenden reprüsentirt. Man hat daher:

$$
\begin{aligned}
\Omega & =(\boldsymbol{\varrho} y) \sqrt{-\Delta^{\prime}}, \\
\frac{\partial \Omega}{\partial \rho_{i}} & =y_{i} \sqrt{-\Delta^{\prime}}=\frac{1}{\mu} y_{i}, \\
\mu & =\frac{1}{\gamma=\overline{\bar{\Delta}^{\prime}}} .
\end{aligned}
$$

Wir schliessen hieraus:

Das Geschlecht der Fläche, welche von den vierpunktigen Tangenten gebildet wird, ist gleich:

$$
P=4 n-13 .
$$

Die Zahl $P$ erniedrigt sicls jedesmal um eine Einheit, wern $\Delta^{\prime}=0$ eine Doppelwurzel hat. Für Flächen, welche einem tinearen speciellen Complexe $\gamma$ angehören, ist insbesondere $P$ immer gleich Null, wie sich auch daraus ergiebt, dass:

$$
\Delta^{\prime}=-\frac{1}{(\varphi \gamma)^{2}}\left[\frac{\partial^{3} \varphi}{\partial \lambda^{3}} \frac{\partial^{3} \varphi}{\partial \lambda^{2} \partial \varphi} \frac{\hat{\partial}^{3} \varphi}{\partial \lambda \partial \mu^{2}} \frac{\partial^{3} \varphi}{\partial \mu^{3}} \gamma \varrho\right]^{2} .
$$

Fügt man den Gleichungen (11) noch die folgende:

$$
a_{y} b_{x}-a_{x} b_{y}=0
$$

hinzu, so erhält man durch Elimination der $y$ eine Gleichung in $\lambda$, welche sich auf den Grad der Curve vierpunktiger Berührung bezieht. Wie die Abzählung lehrt, ist der Grad derselben

$$
5 n-12 \text {. }
$$

Anch diese Curve ist im Allgemeinen hyperelliptisch, ihr Geschlecht das nämliche, wie das der Fläche der vierpunktigen Tangenten, deren Erzeugenden ihre Pankte eindeutig zugeordnet sind. Sie kann ferner im Allgemeinen keine Spitzen haben, da auf jeder Erzeugenden der gegebenen Fläche nur zwei Punkte derselben liegen. Die Singularitäten der Curve viefpunktiger Berührung lassen sich daher ohne weiteres angeben. Man erhält z. B. für den Rang derselben die Zahl 
Die Punkte fünfpunktiger Berührung endlich sind bestimmt durch die Gleichung:

$$
\left(\Omega: \frac{\lambda^{4} \varphi}{\partial \lambda^{4}} \frac{\partial^{4} \varphi}{\partial \lambda^{3} \partial \mu} \frac{\partial^{4} \varphi}{\partial \lambda^{2} \partial \mu^{2}} \frac{\partial^{4} \varphi}{\partial \lambda \partial \mu^{3}} \frac{\partial^{4} \varphi}{\partial \mu^{4}}\right)=0 .
$$

Die Zahl der fünfuntitigen Tangenten ist daher $10(n-4)$.

Die letzthin bestimmten Singularitäten werden ersichtlich durch das Auftreten von Doppelerzeugenden nicht beeinflusst. Für eine windschiefe Fläche dritten Grades ist beispielsweise die Curve vierpunktiger Berührung rom dritten Grade, sie besteht bekanntlich aus einer doppelten und einer einfachen Leitgeraden. Für die windschiefen Flächen vierten Grades $p=0$ ist diese Curve, sowie die von den vierpunktigen Tangenten gebildete Fläche achter Ordnung. Erst die Flächen fünfter Ordnang enthalten zehn Punkte fünfpunktiger Berührung, ihre Doppelcurve einen dreifachen Punkt u. s. w.

\section{XII.}

Fortsetzung. Ueber windschiefe Flächen vom Geschlechte Null, welche linearen Complexen angehören ${ }^{*}$ ).

Die Coefficienteu gleich hoher Potenzen ron $\lambda$ in den Functionen $\varphi$ kann man als Coefficienten der Gleichungen von ebenso viel linearen Complexen auffassen. Wir nennen sie die Fundamentalcomplexe der Fläche, weil ihre Beschaffenheit charakteristisch ist für das Verhalten der letzteren. Zwischen den Invarianten und simultanen Invarianten*:) der Fundamentalcomplexe finden dann diejenigen Relationen statt, welche das identische Verschwinden von $\left(\varphi^{2}\right)=0$ erfordert. Unter ihnen befinden sich immer zwei specielle lineare Complexe, deren Coefficienten die von $\lambda^{\circ}$ und $\lambda^{n}$ sind, ihre $A x \in n$ sind zugleich diejenigen Erzengenden der Fläche, welche den Werthen $\lambda=0, \lambda=\infty$ entsprechen. Die Fläche degenerirt in eine ebene Curve $x^{\text {ter }}$ Classe oder einen Kegel $n^{\text {ter }}$ Ordnung (rom Gesehlecht Null), wenn je zwei Erzeugende sich schneiden. Dazu ist erforderlich, dass alle Fundamentalcomplexe speciell sind und mit einander in Inrolution liegen, $d$. h. dass ihre Axen entweder in einer Ebene liegen oder durch einen gemeinschaftlichen Punkt gehen ***).

Ebene Curven und Kegel beliebigen Geschlechtes lassen übrigens immer, wie wir hier noch bemerken, die folgende Darstellung in Linien-

*) Cremona, Rappresentazione di una classe di superficie gobbe sopra un piano e determinazione delle loro curve assintotiche. Ann. di Mat. Ser. II, t. 1, S. 248 .

**) Klein, Math. Ann. Bd. II, S. 201.

***) Der Begriff der Involution linearer Complexe ist von Herrn Klein erärtert Math. Ann. Bd. II, S. 201. 
coordinaten zu: Sind $\alpha, \beta, \gamma$ drei sich gegenseitig schneidende Gerade, so dass: .

$$
\begin{array}{ll}
\left(\alpha^{2}\right)=0, & \left(\beta^{2}\right)=0, \quad\left(\gamma^{2}\right)=0, \\
(\alpha \beta)=0, & (\beta \gamma)=0, \quad(\gamma \alpha)=0,
\end{array}
$$

so repräsentirt $\lambda \alpha+\mu \beta+\nu \gamma$ immer eine Gerade, welche $\alpha \beta \gamma$ ebenfalls schneidet. Eine homogene Gleichung $n^{\text {len }}$ Grades zwischen $\lambda \mu \nu$ bedeutet daher entweder eine Curve $n^{\text {ter }}$ Classe oder einen Kegel $n^{\text {ter }}$ Ordmung, dessen Erzengende durch

vorgestellt werden.

$$
\varrho y=\lambda \alpha+\mu \beta+\nu \gamma
$$

Wenn ein linearer Complex $\gamma_{x}$ mit allen Fundamentalcomplexen in Involution liegt, so gehören alle Erzeugenden der Fläche diesem Complexe an. Ist derselbe insbesondere speciell, so ist die Axe $\gamma$ desselben eine Leitlinie der Fläche. In diesem Falle bestehen die Gleichungen:

$$
\begin{aligned}
(\gamma \varphi)=0, \quad\left(\gamma \frac{\partial \varphi}{\partial \lambda}\right) & =0, \quad\left(\gamma \frac{\partial^{2} \varphi}{\partial \lambda^{2}}\right)=0 \cdots \text { u. s. w. } \\
\left(\gamma^{2}\right) & =0 .
\end{aligned}
$$

Zieht man eine Gerade $z$, welche $\gamma$ schneidet, so ist $\gamma+\rho z$ ein willkührlicher Strahl des Büschels $(\gamma z)$. Die Erzengendep, welche denselben treffen, sind bestimmt durch:

$$
(\gamma \varphi)+\varphi(z \varphi)=0 \text {, }
$$

oder durch $(z \varphi)=0$. Sie sind also ganz unabhängig ron $\rho, \mathrm{d}, \mathrm{h} . \mathrm{k}_{\mathrm{i}}$ derselben liegen in der Ebene $(\gamma z)$, während $7_{2}$ durch den Purkt $(\gamma z)$ gehen, wobei die Beziehung

$$
k_{1}+k_{2}=n
$$

stattfindet. Die Gerade $\gamma$ ist also $7_{2}$ - fache Leitlinie der Mläche. Ausserdem existirt noch eine krumme Doppelcurre. Bezeichnet man die Anzahl der Punkte, in denen dieselbe der Leitlinie begegnet, mit $\alpha$, so ist der Grad derselben

$$
\alpha+\frac{k_{2}\left(k_{2}-1\right)}{2} \text {. }
$$

Hat die Fläche $d, r$ Doppel - oder Rückkehrgeneratricen, so besteht die Gleichung:

$$
0=n^{2}-3 n+2-k_{2}\left(k_{2}-1\right)-k_{1}\left(k_{1}-1\right)-2 d-2 r-2 a,
$$

oder:

womit die Zahl $\alpha$ bestimmt ist.

$$
n-1+\alpha+d+r=k_{1} k_{2}
$$

Wenn die Fläche zwei linearen Complexen angehört, so existiren zwei Leitlinien, von denen die eine $k_{1}-$, die andere $k_{z_{2}}$-fach ist und ausserdem keine Doppeleurve. Es ist daher $a=0$, und

$$
n-1+d+r=k_{2} k_{z} \text {. }
$$


Wenn $d$ und $r$ gleich Null sind, so ist immer $k_{1}=1, k_{2}=n-1$.

Wir untersuchen jetzt die Haupttangentencurven solcher Flächen, die zunächst einem linearen Complexe angehören. Die ausgezeichnete algebraische Haupttangentencurve ist in diesem Falle von der Ordnung und Classe $2(n-1)-r$. Auf jeder Erzeugenden der Fläche liegen zwei Punkte derselben. Thre Tangenten müssen sich daher als Functionen ron $\lambda$ darstellen, welche eine quadratische Irrationalität enthalten. Wir haben damit den Satz:

Die algebraische Haupttangentencurve auf einer windschiefen Fläche vom Geschlechte Null, die einem linearen Complexe angehört, ist im Allgemeinen hyperelliptisch.

Die Darstellung der sämmtlichen Tangenten der Haupttangentencurve als functionen von $\lambda$ exhält man auf demselben Wege, welcher bei der Betrachtung der Fläche der vierpunktigen Tangenten eingeschlagen wurde. Gehört die Fläche dem linearen Complexe $\gamma_{x}=0$ an, so sind die Tangenten $y$ der genannten Curve charakterisirt durch:

$$
\left\{\begin{array}{l}
\left(y \frac{\partial^{2} \varphi}{\partial \lambda^{2}}\right)=0 \\
\left(y \frac{\partial^{2} \varphi}{\partial \lambda}\right)=0 \\
\left(y \frac{\hat{\partial}^{2} \varphi}{\partial \mu^{2}}\right)=0 \\
(\gamma y)=0, \\
\left(y^{2}\right)=0 .
\end{array}\right.
$$

Um die Auflösung dieser Gleichungen zu bewerkstelligen, setzen wir

Alsdann ist wieder

$$
\Omega=\left(\frac{\partial^{2} \varphi}{\partial \lambda^{2}} \frac{\partial^{2} \varphi}{\partial \lambda \partial \mu} \frac{\partial^{2} \varphi}{\partial \mu^{2}} \gamma y \varrho\right)
$$

$$
y_{i}=\mu \frac{\partial \Omega}{\partial e_{i}}
$$

und die $y$ proportional den Unterdeterminanten aus den Coefficienten der Gleichungen XI. (12). Zur Bestimmung von $\mu$ hat man:

$$
\Omega^{2}=\left|\begin{array}{cccccc}
B_{11} & B_{12} & B_{13} & 0 & 0 & a_{1} \\
B_{21} & B_{22} & B_{23} & 0 & 0 & a_{2} \\
B_{31} & B_{32} & B_{33} & 0 & 0 & a_{3} \\
0 & 0 & 0 & \left(\gamma^{2}\right) & 0 & a_{1} \\
0 & 0 & 0 & 0 & 0 & \varrho y \\
a_{1} & a_{2} & a_{3} & a_{4} & \varrho y & \varrho^{2}
\end{array}\right|=-(\varrho y)^{2}\left(\gamma^{2}\right) \Delta .
$$

Es bedeuten hier die $B_{11} B_{12} \cdots$ die Ausdrücke

$$
\Sigma\left(\frac{\partial^{2} \varphi_{i}}{\partial \lambda^{2}}\right)^{2}, \quad \Sigma \frac{\partial^{2} \varphi_{i}}{\partial \lambda^{2}} \frac{\partial^{2} \varphi_{i}}{\partial \lambda \partial \mu}, \cdots,
$$


$\Delta$ die Determinante:

$$
\left\{\begin{array}{lll}
B_{11} & B_{12} & B_{13} \\
B_{21} & B_{22} & B_{23} \\
B_{31} & B_{32} & B_{33}
\end{array}\right.
$$

Es ist aber $\Delta \mathrm{im}$ Wesentlichen identisch mit der dritten Patenz von $\left(\frac{\partial \varphi^{2}}{\partial \lambda}\right)$ oder $\Psi(\lambda \lambda)$. Denn man hat

$$
\left(\varphi \frac{\partial \varphi}{\partial \lambda} \frac{\partial^{2} \varphi}{\partial \lambda^{2}} \gamma y \varphi\right)=\frac{1}{n(n-1)^{2}}\left(\frac{\partial^{2} \varphi}{\partial \lambda^{2}} \frac{\partial^{2} \varphi}{\partial \lambda \partial \mu} \frac{\partial^{2} \varphi}{\partial \mu^{2}} \gamma y \varphi\right)
$$

woraus, wenn man auf beiden Seiten zum Quadrat erhebt:

Es ist daher

$$
(\Psi \lambda \lambda)^{3}=\frac{1}{n^{2}(n-1)^{4}} \Delta \text {. }
$$

$$
\mu=\frac{1}{(\Psi \lambda \lambda) n(n-1)^{2} V-(\bar{\psi} \lambda \bar{\lambda})\left(\gamma^{2}\right)} .
$$

Der Ausdruck $\Psi \lambda \lambda$ ist daher entscheidend für das Geschlecht der Haupltangentencurve, Man Kann ithn die Discriminante der Fläche nennen. Bezeichnet man die Zahl der Rückkehrgeneratricen derselben durch $x$, die der zusammengerückten singulären Erzeugenden durch $x$, so ist demnach das Geschlecht der Haupttangentencurve

$$
P=n-3-r-x \text {. }
$$

Man gewinnt daraus die folgenden Zahlen:

$$
\begin{aligned}
& M=N=2(n-1)-r, \\
& R=6(n-2)-4 r-2 x, \\
& \Omega=10 n-8 r-6 x-28 .
\end{aligned}
$$

Von den stationären Tangenten $\Omega$ liegen $2 n-4-2 r$ in den Cuspidalpunkten der Fläche, die übrigen $8(n-3)-6(x 7-x)$ beziehen sich auf die Berührungspunkte der Curve vierpunktiger Berührung.

Wenn die Fläche zwei linearen speciellen Complexen mit windschiefen, nicht zusammenfallenden Axen $\gamma^{\prime} \gamma^{\prime}$ angehört, so hat man zur Bestimmung einer beliebigen Haupttangentencurve die drei ersten Gleichungen (1) und:

$$
\begin{aligned}
\left(y \gamma^{\prime}\right)+\rho\left(\gamma^{\prime \prime} y\right) & =0, \quad\left(y^{2}\right)=0, \\
\left(\gamma^{\prime 2}\right) & =0, \quad\left(\gamma^{\prime \prime 2}\right)=0,
\end{aligned}
$$

zu nehmen, in denen $\varrho$ einen willkührlichen Parameter vorstellt, welcher je eine Haupttangentencurve individualisirt. An Stelle derselben kann man, um eine einfachere Lösung als die durch die Gleichungen XI. (12) gebotene zu erhalten, das folgende System benutzen, in welchem $\mu$ wieder gleich eins zu setzen ist. 


$$
\left\{\begin{array}{l}
\left(y-\frac{\partial^{3} \varphi}{\partial \lambda^{3}}\right)=-1 \\
\left(y \frac{\partial^{3} \varphi}{\partial \lambda^{2} \partial \mu}\right)=\lambda \\
\left(y \frac{\partial^{3} \varphi}{\partial \lambda \partial \mu^{2}}\right)=-\lambda^{2} \\
\left(y \frac{\partial^{3} \varphi}{\partial \lambda^{3}}\right)=\lambda^{3} \\
\left(\gamma^{\prime} y\right)=-\varrho \omega \\
\left(\gamma^{\prime \prime} y\right)=\omega \\
\left(y^{2}\right)=0
\end{array}\right.
$$

Die Elimination der $y$ aus diesen sieben Gleichungen liefert die folgende zur Bestimmung von $\omega$ :

$$
\left|\begin{array}{ccccccc}
0 & -1 & \lambda & -\lambda^{2} & \lambda^{3} & -\varrho \omega & \omega \\
-1 & A_{11} & A_{12} & A_{13} & A_{14} & 0 & 0 \\
\lambda & A_{21} & A_{22} & A_{23} & A_{24} & 0 & 0 \\
-\lambda^{2} & A_{31} & A_{32} & A_{33} & A_{34} & 0 & 0 \\
\lambda^{3} & A_{41} & A_{42} & A_{43} & A_{44} & 0 & 0 \\
-\rho \omega & 0 & 0 & 0 & 0 & 0 & \left(\gamma^{\prime} \gamma^{\prime \prime}\right) \\
\omega & 0 & 0 & 0 & 0 & \left(\gamma^{\prime} \gamma^{\prime \prime}\right) & 0
\end{array}\right|=0 \text {. }
$$

Die $A_{i k}$ sind Functionen $2(n-3)^{\text {ten }}$ Grades in $\lambda$. Bezeichnet man die Determinante der $A_{i k}$, gerändert mit $-1 \lambda-\lambda^{2} \lambda^{3}$ durch $\Delta^{\prime \prime}$, so geht (3) über in

$$
\Delta^{\prime \prime}\left(\gamma^{\prime} \gamma^{\prime \prime}\right)+2 \rho \omega^{2} \Delta^{\prime}=0
$$

Die Determinante $\Delta^{\prime}$ ist nach $\S$ XI. ein vollständiges Quadrat, während die Determinante $\Delta^{\prime \prime}$ im Wesentlichen mit $\Delta$ oder $\Psi \lambda \lambda$ identisch sein muss. In der That hat man:

$$
\left(\frac{\partial^{2} \varphi}{\partial \lambda^{2}} \frac{\partial^{2} \varphi}{\partial \lambda \partial \mu} \frac{\partial^{2} \varphi}{\partial \mu^{2}} \gamma^{\prime} \gamma^{\prime \prime} y\right)=\frac{-1}{(n-2)^{3}}\left|\begin{array}{ccc}
\frac{\partial^{3} \varphi_{i}}{\partial \lambda_{1}{ }^{3}} & -1 & 0 \\
\frac{\partial^{3} \varphi_{i}}{\partial \lambda^{2} \partial \mu} & \lambda & 0 \\
\frac{\partial^{3} \varphi_{i}}{\partial \lambda \partial \mu^{2}}-\lambda^{2} & 0 \\
\frac{\partial^{3} \varphi_{i}}{\partial \mu^{3}}+\lambda^{3} & 0 \\
\gamma_{i}^{\prime} & 0 & 0 \\
\gamma_{i}^{\prime \prime} & 0 & 0 \\
y & 0 & 0 \\
0 & 0 & 1
\end{array}\right|,
$$


ergiebt.

$$
\Delta=-\frac{1}{(n-2)^{6}} \Delta^{\prime \prime}
$$

Die sechs ersten Gleichungen (2) geben dann unmittelbar die Coordinaten $y$, ausgedrückt durch rationale Functionen von $\lambda$ und $\boldsymbol{\omega}$, wobei

$$
\omega=(n-2)^{3}(n-1)^{2} n \psi \lambda \lambda \sqrt{\frac{\psi_{\lambda} \lambda \overline{\lambda\left(\gamma, \gamma_{1}\right)}}{2 Q \Delta^{\prime}}}
$$

und damit die Darstellung aller der developpabelen Flächen, deren Rückkehrkanten die Haupttangenten sind, abhängig ron dem willkührlichen Parameter $\varrho$.

Die Singularitäten der Happttangentencurven sind durch die obigen Zahlen für $M, N, P, R, \Omega$ gegeben. Nur beziehen sich jetzt die stationären Tangenten, welche nicht in die Cuspidalpunkte fallen, paarweise auf die hyperbolischen Erzeugenden.

Windschiefe Flächen $n^{\text {ten }}$ Grades $p=0$, welche einem Büschel linearer Complexe mit discreten Directricen angelören, haben also $4(n-3)-3(r+x)$ hyperbotische Erzengende.

Es ist endlich noch der Fall zu erörtern, wo die Directricen coincidiren. Die sämmtlichen Haupttangentencurven sind dann gegeben durch die Gleichungen:

$$
\left\{\begin{array}{l}
\left(y \frac{\partial^{2} \varphi}{\partial \lambda^{2}}\right)=0, \\
\left(y \frac{\partial^{2} \varphi}{\partial \partial \partial \mu}\right)=0, \\
\left(y \frac{\partial^{2} \varphi}{\partial \mu^{2}}\right)=0, \\
\left(y \gamma^{\prime}\right)+\rho\left(\gamma^{\prime \prime} y\right)=0, \\
\left(y^{2}\right)=0 .
\end{array}\right.
$$

wo $\left(\gamma^{\prime 2}\right)=0,\left(\gamma^{\prime} \gamma^{\prime \prime}\right)=0$.

In Folge dessen ist für jeden Werth von $\lambda$ eine der Lösungen von $y$

$$
y_{i}=\gamma_{i}^{\prime} \text {. }
$$

Auf jeder Erzeugenden liegt daher nur ein Punkt der Haupttangentencurve. Es ist damit der Satz bewiesen:

Die Haupttangentencurven der windschiefen Flächen $p=0$ mit zusammenfallenden Leitlinien sind algebraische Ourven vam Geschlechte Null*).

Dasselbe zeigt auch die Untersuchung der Discriminante. Man hat nämlich:

$$
\left(\frac{\partial^{2} \varphi}{\partial \lambda^{2}} \frac{\partial^{2} \varphi}{\partial \lambda \partial \mu} \frac{\partial^{2} \varphi}{\partial \mu^{2}} \gamma^{\prime} \gamma^{\prime \prime} \varrho\right)^{2}=-\left(\gamma^{\prime} \varrho\right)^{2}\left(\gamma^{\prime \prime 2}\right) \Delta,
$$

so dass $\Delta$ ein vollständiges Quadrat ist.

*) Cremona a. a. 0 .

Mathematischo Annalen. VIII. 
A. Voss.

Bezeichnet man mit $k_{1}$ die kleinere der beiden Zahlen, welché die Multiplicität der beiden unendlich nahen Leitlinien ausdrücken, so ist Ordnung und Classe der Haupttangentencurven (Räckkehrgeneratricen nicht vorausgesetzt)

woraus

$$
M=N=2(n-1)-k_{1},
$$

$$
\begin{aligned}
& R=2 M-2=4 n-6-2 k_{1}, \\
& \Omega=4 n-10-2 k_{1} .
\end{aligned}
$$

Die windschiefen Flächen $n^{\text {ten }}$ Grades $p=0$ mit zwei unendlich nahen $k_{1}$ - wnd $k_{2}$-fachen Leitlinien $k_{1} \leq k_{2}$ luaben $4 s_{4}-10-2 k_{i_{1}}$ hyperbolische Erzeugende.

Man kann, wie wir zum Schluss noch bemerken wollen, ähnliche Formeln für die Singularitäten der Haupttangentencurven auch für windschiefe Flächen beliebigen Geschlechts, die Inearen Complexen angehören, aufstellen.

Die Fläche gehöre einem linearen Complex an, ihr Grad sei n, der Grad der Doppelcurve $D$, die Zahl der Doppel-, resp. Rückkehrgeneratricen $d, r$. Das Geschlecht der Fläche ist

$$
2 p=(n-1)(n-2)-2 d-2 v-2 D \text {. }
$$

Für Ordnung und Classe der Haupttangenteneurve ergiebt sich demnach:

$$
M=N=n(n-1)-2 d-3 r-2 D=2 p+2 n-2-r .
$$

Den Rang der Haupttangentencurre bestimmt man auf folgende Weise. Die sämmtlichen Haupttangenten bilden ein Strahlensystem, dessen Ordnung and Classe

$$
i=3 n(n-2)-6 d-8 r-6 D .
$$

Die Hauptangenten, welche dem linearen Complexe $C$ angehören und eine willkührliche Gerade $z$ schneiden, schneiden zugleich eine zweite Gerade, die conjugirte Polare von $z$ in Bezug auf $C$. Es bilden aber die Haupttangenten, welche $z$ schneiden, eine windschiefe Fläche der Ordnung $2 i$. Der Rang der Haupttangentencurve ist daher:

$$
R=6 n(n-2)-12 d-16 r-12 D .
$$

Das Geschlecht der Haupttangentencurve bestimmt sich aus der Formel

$$
\begin{aligned}
& R=2 P+2(N-1), \\
& P=3 n(n-2)-6 d-8 r-6 D-N+1, \\
& P=4 p+n-3-r .
\end{aligned}
$$

Daraus ergiebt sich die Zahl der stationären Tangenten 


$$
\begin{aligned}
\Omega & =3 R-4 N \\
& =28 p+10 n-8 r-28 .
\end{aligned}
$$

Es ist aber die Zahl der singulären Erzeugenden der windschiefen Hläche fermer:

$$
4 p+2 n-4-2 r \text {. }
$$

Subtrahirt man diese Zahl von $\Omega$, so erhält man endlich als Zahl der Berïhrungspuntite dex Curve vierpunktiger Berührung mit den Exzeugenden:

$$
8(n-3)+6(4 p-r) \text {. }
$$

Die entwiclelten Formeln bleiben anch dann noch gültig, wenn die Fläche zwei speciellen linearen Complexen mit discreten Axen angehört. Solche Flächen besitzen daher

$$
4(n-3)+3(4 p-r)
$$

lyperbolische Eraeugende.

Für Flächen mit zwei unendlich nahen, $k_{1}-, k_{2}$-fachen Leitlinien, sowie d, $r$ Doppel und Rücklehrgenesatricen gelten dagegen die folgenden Formeln:

$$
\begin{aligned}
P=p & =k_{1} k_{2}-d-r-n+1, \\
N=\not N & =2 p-r+2 n-2-k_{1}, \\
\Omega & =10(p-1)+4 n-2 r-2 k_{1},
\end{aligned}
$$

in welchen $\Omega$ die Zahl der hyperbolischen Erzeugenden angiebt*).

\section{XIII.}

\section{Developpabele Flächen vom Geschlechte Null.}

Die windschiefe Fläche, deren Erzeugende durch die Gleichungen

$$
\varrho x_{i}=\varphi_{i}
$$

dargestellt sind, ist abwickelbar, wenn je zwei consecutipe Erzeugende sich schneiden. Es müssen also die beiden Gleichungen:

$$
\begin{aligned}
& \left(\varphi^{2}\right)=0, \\
& \left(\frac{\partial \varphi^{2}}{\partial \lambda}\right)=0
\end{aligned}
$$

Identitäten sein. In Folge dessen hat man die weiteren Gleichungen:

$$
\left(\varphi \frac{\partial \varphi}{\partial \lambda}\right)=0,\left(\varphi \frac{\partial^{2} \varphi}{\partial \lambda^{2}}\right)=0,\left(\varphi \frac{\partial^{3} \varphi}{\partial \lambda^{3}}\right)=0,\left(\frac{\partial \varphi}{\partial \lambda} \frac{\partial^{2} \varphi}{\partial \lambda^{2}}\right)=0 \text { u. s.w. }
$$

Bei Anwendung homogener Parameter $\lambda \mu$ kann man ihnen noch die folgenden:

) Inzwischen habe ich die Ordnung der Curve vierpunktiger Berührang u. s. w. auf einer beliebigen (windschiefen) Fläche nier Oxdnung bestimmt, worauf ich bei einer anderen Gelegenheit zurückkommen werde. 


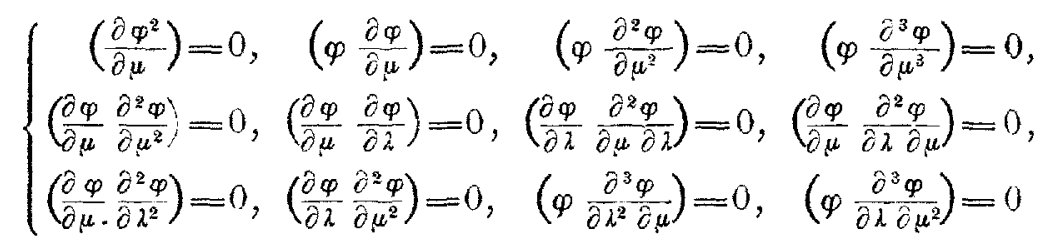

hinzufügen.

Die Gleichungen (1) sagen aus, dass jede Erzengende von $n-4$ anderen geschnitten wird. Bezeichnet man nämlich mit $\lambda$ den Parameter von $x$, mit $\lambda+x$ den einer zweiten Erzeugenden, welche $x$ schneidet, so ergiebt die Gleichung:

$$
\left(\varphi_{2} \varphi \lambda+k\right)=0=\left(\varphi \lambda^{2}\right)+\frac{x}{1}\left(\varphi \frac{\partial \varphi}{\partial \lambda}\right)+\frac{\lambda^{2}}{2 !}\left(\varphi \frac{\partial^{2} \varphi}{\partial \lambda^{2}}\right)+\frac{x^{3}}{3 !}\left(\varphi \frac{\partial^{3} \varphi}{\partial \lambda^{3}}\right)+\cdots
$$

ausser den vier Wurzeln $x=0$ noch $n-4$ andere, welche sich auf die auf $x$ liegenden Punkte der Doppelcurve beziehen. Es giebt daher eine bestimmte Zahl ron Erzeugenden, für welche nur $n-5$ Wurzeln dieser Gleichung von Null verschieden sind. Sie sind bestimmt durch

$$
\left(\varphi \frac{\hat{c}^{4} \varphi}{\hat{\partial} \hat{\lambda}^{4}}\right)=0
$$

und beziehen sich auf die stationären und Wendungspunkte der Rückkehreurve der developpabeln Wäche.

Bezeichnet man die $\mathrm{Zahl}$ dieser beiden Singularitäten respective durch $\alpha, \beta$, so zeigt die letzte Gleichung, dass

$$
\alpha+\beta=2 n-8 \text {. }
$$

Die Zahlen $\alpha, \beta$ dürfen nicht mehr, wie in den früheren Betrachtungen einander gleich gesetzt werden, weil eine developpable Fläche im Allgemeinen kein in sich dualistisches Gebilde ist."

Bezeichnet man den Grad der Rückkehrcurve durch $M$, so ist der Rang der Developpabeln

$$
2(n-1)-M \text {. }
$$

Diese Zahl stellt zugleich die Classe des ebenen Schnittes oder die Classe der Rückkehreurve vor. Man hat daher:

$$
\begin{aligned}
M+N & =2(n-1), \\
p & =0, \\
P & =n .
\end{aligned}
$$

Wir schliessen mit der folgenden Bemerkung. Der dereloppabeln Fläche ist eine zweite Fläche $(n-1)^{\text {ten }}$ Grades

$$
\rho y_{i}=\frac{\partial \varphi_{i}}{\partial \lambda}
$$

eindeutig zugeordnet. . Aus den Gleichungen (1) ersieht man unmittelbar, dass je drei consecutive Erzeugende derselben: 


\section{$y, \quad y+d y, \quad y+2 d y+d^{2} y$}

von der Erzeugeuden $x$ geschnitten werden. Die Fläche $y$ hat daher die Rückkehrcurve von $x$ zur Haupttangentencarve, ihre Erreugenden schneiden die letztere und verlaufen zugleich in der jedesmaligen Schmiegungsebene derselben. Denselben Charakter besitzt aber auch jede andere Fläche, deren Erzengende $z$ durch die Gleichung

oder auch

$$
\varrho z_{i}=\alpha x_{i}+\beta \frac{\partial \varphi_{i}}{\partial \lambda}
$$

$$
\varrho z_{i}=a \frac{\partial \varphi_{i}}{\partial \lambda}+b \frac{\partial \varphi_{i}}{\partial u}
$$

definirt werden. Die Grössen $a, b$ könmen dabei völlig willkührliche Functionen von $\lambda$ sein. Nimmt man sie insbesondere als constant an, so hat man den Satz:

Einer jeden abwickelbaren Fläche vom Geschlechte Null $n^{\text {ten }}$ Grades lässt sich ein "Büschel" windschiefer Flächen $n-1^{\text {ten }}$ Grades von gleichem Geschlechte zwordnen, welche die Rückkehrourve der gegebenen Fläche zur gemeinschaftluchen Hawpttangentencurve haben.

\section{XIV.}

\section{Betrachtung einiger bestimmter windschiefer Flächen.}

Auf die in den vorigen beiden Paragraphen entwickelten Vorstellnngen lässt sich eine Eintheilung der windschiefen Flächen $p=0$ begründen, in welcher die Fundamentalcomplexe eine wesentliche Rolle spielen. Wir ziehen es aber vor, van der weiteren Verfolgung der allgemeinen Betrachtungen zunächst absehend, einige bestimmte Linienflächen zu untersuchen, womit denn zugleich die Rïchtung der liniengeometrischen Methoden überhaupt genauer bezeichnet werden wird.

\section{A. Windschiefe Flächen zweiten Grades.}

Sie sind gegeben durch die Gleichungen

$$
\varrho x_{i}=a_{i}+b_{i} \lambda_{i}+c_{i} \lambda^{2}
$$

wobei

$$
\left(a^{2}\right)=0, \quad(a b)=0, \quad\left(c^{2}\right)=0, \quad(b c)=0, \quad\left(b^{2}\right)+2(a c)=0 .
$$

Die Fläche (1) ist ein Hyperboloid, dessen Erzeugende erster Art die $x$ sind. Die Fundamentalcomplexe

$$
a_{y}=0, \quad b_{y}=0, \quad c_{y}=0
$$

bestimmen dagegen die Erzeugenden zweiter Art. Stellt man dieselben in der Form:

$$
\varrho y_{i}=\alpha_{i}+\beta_{i} \mu+\gamma_{i} \mu^{2}
$$

dar, so ist 

$\left(\alpha^{2}\right)=0$,
$(\boldsymbol{\alpha} \boldsymbol{\beta})=0$,
$\left(\gamma^{2}\right)=0$,
$(\beta \gamma)=0$,
$\left(\beta^{2}\right)+2(\gamma \alpha)=0$

während zwischen den $\alpha \beta \gamma, a b c$ die Bedingungen

stattfinden.

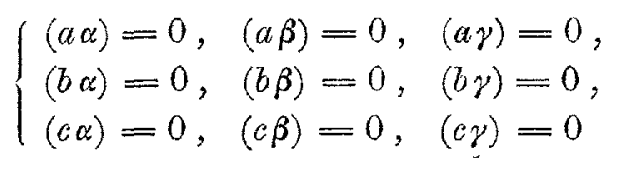

Die Gleichungen (1), (2) vermitteln die Abbildung sämmtlicher Erzeugenden der Fläche auf die ebenen Strahlbüschel $k_{1}+\lambda k_{2}, k_{1}+\mu k_{2}$. Das Doppelverhältniss von vier beliebigen Erzeugenden einer Art $x_{1}$, $x_{2}, x_{3}, x_{4}$, denen die Parameter $\lambda_{1}, \lambda_{2}, \lambda_{3}, \lambda_{4}$ entsprechen, ist daher dasselbe wie das der Stralilen

also gleich

$$
k_{1}+\lambda_{1} k_{2}, k_{1}+\lambda_{2} k_{2}, \quad k_{1}+\lambda_{3} k_{2}, \quad k_{1}+\lambda_{1} k_{2}
$$

$$
\delta=\frac{\lambda_{1}-\lambda_{3}}{\lambda_{2}-\lambda_{3}}: \frac{\lambda_{1}-\lambda_{4}}{\lambda_{2}-\lambda_{4}} .
$$

Man hat andererseits zwischen zwei Erzeugenden $x_{2} x_{k}$ die Beziehung:

$$
\varrho_{i} \varrho_{k}\left(x_{\iota} x_{k}\right)=\left(\lambda_{i}-\lambda_{k}\right)^{2},
$$

woraus, wie .schon in $\$$ II. gefunden wurde:

$$
\delta=\sqrt{\frac{\left(x_{1} x_{3}\right)}{\left(x_{2} x_{3}\right)}: \frac{\left(x_{1} x_{4}\right)}{\left(x_{2} x_{4}\right)} .}
$$

Infolge der Gleichungen (1), (2), (3) ist ferner durch die Gleichungen

$$
\varepsilon z_{i}^{\prime}=x_{i}-\rho^{\prime} y_{i}=a_{i}-\rho^{\prime} \alpha_{i}+\left(b_{i}-\rho^{\prime} \beta_{i}\right) \lambda+\left(c_{i}-\rho^{\prime} \gamma_{i}\right) \lambda^{2}
$$

ein Hyperboloid gegeben, dessen Erzeugende zweiter Art durch

(5) $\varepsilon z_{i}^{\prime \prime}=x_{i}-\varrho^{\prime \prime} y_{i}=a_{i}-\varrho^{\prime \prime} \alpha_{i}+\left(b_{i}-\varrho^{\prime \prime} \beta_{i}\right) \mu+\left(c_{i}-\varrho^{\prime \prime} \gamma_{i}\right) \mu^{2}$

bestimmt sind, wenn zwischen den Parametern $\rho^{\prime} \varrho^{\prime \prime}$ die Gleichung

stattfindet.

$$
\varrho^{\prime} \varrho^{\prime \prime}=-\frac{(a c)}{(\alpha \gamma)}=-\frac{\left(b^{2}\right)}{\left(\beta^{2}\right)}
$$

Durch eine Gleichung zwischen $\lambda$ und $\mu$, etwa $f(\lambda \mu)=0$, werden die Erzeugenden erster und zweiter Art auf eine gewisse Weise mit einander in Beziehung gesetzt. Ist dieselbe vom Grade $m_{1}$ in Bezug auf $\lambda$, vom Grade $m_{2}$ in Bezug auf $\mu$, so schneiden sich die zugehörigen Erzengenden in einer Curve $m_{1}+m_{2}^{\text {ter }}$ Ordnung, welche jeder Erzeugenden erster Art in $m_{2}$, jeder von der zweiten Art in $m_{1}$ Punkten begegnet. Den Discriminanten von $f$ in Bezug auf $\mu \lambda$, welche in Bezug auf diese Grössen von den Graden $2 m_{1}\left(m_{2}-1\right), 2 m_{1}\left(m_{2}-1\right)$ sind, entsprechend, wird jede dieser Curren $2 m_{1}\left(m_{2}-1\right)$ Erzeugenden erster, $2 m_{2}\left(m_{1}-1\right)$ zweiter Art berühren.

Eine bilineare Beziehung zwischen $\lambda, \mu$ stellt daher einen auf dem 
Hyperboloide gelegenen Kegelschnitt vor. Setzt man insbesondere $\lambda=\mu$, so stellen die Gleichungen (4), (5) eine einfach anendliche Schaar von Hyperboloiden vor, welche das gegebene in dem Kegelschnitte $\lambda=\mu$ berihren. Für jedes Hyperboloid hat dabei das Doppelverhältniss dieser beiden Erzeugenden, die von einem beliebigen Punkte des Kegelschnittes auslaufen, zu den entsprechenden Erzeugenden des gegebenen den constanten Werth

$$
\delta=\frac{e^{\prime}}{e^{\prime \prime}} .
$$

Für den besonderen Werth $\rho^{\prime 2}=-\frac{\left(b^{2}\right)}{\left(\beta^{2}\right)}$ geben die Gleichungen (4), (5) die Gleichung des Berührungskegelschnittes, sowie die des längs desselben berührenden Kegels.

\section{B. Windschiefe Flächen dritten Grades.}

Für dieselben ist

$$
\begin{gathered}
\omega x_{i}=a_{2}+\lambda \vec{b}_{\imath}+\lambda^{2} c_{2}+\lambda^{3} d_{t} \\
\left(a^{2}\right)=0, \quad(a b)=0,\left(d^{2}\right)=0,(d c)=0, \\
\left(b^{2}\right)+2(a c)=0, \quad\left(c^{2}\right)+2(b d)=0, \\
(a d)+(b c)=0 .
\end{gathered}
$$

Die Fläche hat zwei singuläre Erzeugende, bestimmt durch die Gleichung

$$
\left(b^{2}\right)+4(b c) \lambda+\lambda^{2}\left(4\left(c^{2}\right)+6(b d)\right)=0 .
$$

Dieselben coincidiren, wenn die Discriminante

$$
4(b c)^{2}-\left(b^{2}\right)\left(d^{2}\right)
$$

verschwindet. Diesen Fall ausgeschlossen, kann man den Parameter $\lambda$ so gewählt denken, dass die Werthe $\lambda=0, \lambda=\infty$ die singulären Erzeugenden selbst darstellen. Unter dieser Voraussetzung bestehen die Identitäten

$$
\begin{gathered}
\left(a^{2}\right)=0, \quad(a b)=0, \quad\left(b^{2}\right)=0,\left(d^{2}\right)=0,(b d)=0,(d c)=0, \quad(a c)=0, \\
(a d)+(b c)=0 .
\end{gathered}
$$

Die Fläche gehört unendlich vielen linearen Complexen an, deren Coefficienten $\gamma$ bestimmt sind durch die Gleichungen:

$$
(\gamma a)=0, \quad(\gamma b)=0,(\gamma c)=0, \quad(\gamma d)=0 .
$$

Der Voraussetzung $4(b c)^{2}-\left(b^{2}\right)\left(d^{2}\right) \leqq 0$ entsprechend befinden sich unter ihnen immer zwei verschiedene specielle, deren Axen durch $\gamma^{\prime}, \gamma^{\prime \prime}$ bezeichnet werdep mögen. Dieselben bilden zwei Leitlinien der Fläche, von denen die eine einfach, die andere doppelt ist. Die Fläche ist auf ein Tetraeder bezogen, dessen Kanten, zu gegenüberliegenden Paaren geordnet, $a d, b c, \gamma^{\prime} \gamma^{\prime \prime}$ sind. Die Kante $\gamma^{\prime}$, welche durch den Schnitt ron $a b, c d$ geht, ist die Doppelgerade der Fläche. 
Die Gleichung $\Psi \lambda \lambda^{\prime}=0$ des $\$$ XI. (5) nimmt die Form $\lambda+\lambda_{1}=0$ an. Je zwei Parameterwerthe $\lambda$ and $-\lambda$ bestimmen daher Erzeugende, die sich in einem Punkte von $\gamma^{\prime}$ schneiden. Bezeichnet man ihre Coordinaten durch $x, x^{\prime}$, so ist $x+\varrho x^{\prime}$ ein beliebiger Strahl des Büschels $x x^{\prime}$. Soll derselbe gleichreitig $a$ und $c$ schneiden, so ist $1-\varrho=0$ zu setzen, während dem Strahle, welcher $b$ und $d$ schneidet $1+\rho=0$ entspricht. Es ist damit der folgende Satz bewiesen:

Die beiden Erzeugenden, welche sich in einem Punkte der Doppelgeraden kreuzen, schneiden die einfache Leitlinie in zwei Punlten, die harmonisch sind zu den Puntiten, in denen die letztere von den singulären Erzeugenden getroffen wird.

Man hat ferner:

Es ist daher

$$
\begin{aligned}
& \rho a_{x}=(a d) \lambda^{3}, \\
& \varrho b_{x}=(b c) \lambda^{2}, \\
& \rho c_{x}=(b c) \lambda, \\
& \varrho d_{x}=(a d) .
\end{aligned}
$$

$$
a_{x} d_{x}-b_{x} c_{x}=0
$$

Die Fläche gehört also nach $\$ 1$ : einem speciellen Reye'schen Complexe an.

Die Haupttangenten derselben sind ferner bestimmt durch die Gleichungen :

$$
\begin{aligned}
3 a_{y}+\lambda b_{y} & =0, \\
b_{y}+\lambda c_{y} & =0, \\
c_{y}+3 \lambda d_{y} & =0 .
\end{aligned}
$$

Schreibt man dieselben in der form

so ist

$$
\left\{\begin{array}{l}
a_{y}=-\lambda^{3} \\
b_{y}=3 \lambda^{2} \\
c_{y}=-3 \lambda \\
a_{y}=1
\end{array}\right.
$$

$$
9 a_{y} d_{y}-b_{y} c_{y}=0 \text {. }
$$

Die Hauptlangenten der Fläche schneiden also das durch die Fundamentalcomplexe gebildete Tetraeder in constantem Doppelverhälniss.

Die Gleichung einer beliebigen Haupttangentencurve erhält man nach XII. (5), indem man den Gleichungen (7) die folgenden

$$
\begin{aligned}
& \gamma_{y}^{\prime}=-\rho \omega, \\
& \gamma_{y}^{\prime \prime}=\omega, \\
& \left(\gamma^{2}\right)=0
\end{aligned}
$$

hinzufügt. Den Werthen 


$$
\begin{aligned}
& \Delta^{\prime}=(b c)^{1}, \\
& 0-\lambda^{3} 3 \lambda^{2}-3 \lambda \quad 1 \\
& \begin{array}{lllll}
-\lambda & 0 & 0 & 0 & (a d)
\end{array} \\
& \Delta^{\prime \prime}=\begin{array}{lllll}
3 \lambda^{2} & 0 & 0 & (b c) \quad 0 & =16(b c)^{3} \lambda^{3}
\end{array} \\
& \begin{array}{lllll}
-3 \lambda & 0 & \text { (bo) } & 0 & 0
\end{array} \\
& 1 \text { (ad) } 0000
\end{aligned}
$$

entsprechend hat man daher

$$
\rho \omega^{2}=-8 \frac{\left(\gamma^{\prime} \gamma^{\prime \prime}\right)}{(b c)} \lambda^{3}
$$

Unter Voraussetzung dieses Werthes ron $\omega$ sind die $y$ die Unterdeterminanten nach $\mu_{i}$ der folgenden Determinante:

so dass:

$$
\Omega=\begin{array}{ccccccc}
\mu_{4} & \mu_{3} & \mu_{3} & \mu_{4} & \mu_{5} & \mu_{6} & 0 \\
a_{1} & a_{2} & a_{3} & a_{4} & a_{5} & a_{6} & -d^{3}, \\
b_{1} & b_{2} & b_{3} & b_{4} & b_{5} & b_{6} & 3 \lambda^{2} \\
c_{4} & c_{2} & c_{3} & c_{4} & c_{5} & c_{6} & -3 \lambda^{2} \\
d_{4} & d_{2} & d_{3} & d_{4} & d_{5} & d_{6} & 1 \\
\gamma_{4}^{\prime} & \gamma_{2}^{\prime} & \gamma_{3}^{\prime} & \gamma_{4}^{\prime} & \gamma_{5}^{\prime} & \gamma_{6}^{\prime} & -\varrho \omega \\
\gamma_{1}^{\prime \prime} & \gamma_{2}^{\prime \prime} & \gamma_{3}^{\prime \prime} & \gamma_{4}^{\prime \prime} & \gamma_{5}^{\prime \prime} & \gamma_{6}^{\prime \prime} & \omega
\end{array}
$$

(9)

$$
y_{i} \equiv \frac{\partial \Omega}{\partial \mu_{i}} \text {. }
$$

Die Determinante $\Omega$ reducirt man durch Multiplication mit

$$
\left(a b c d \gamma^{\prime} \gamma^{\prime \prime}\right)=\left(\gamma^{\prime} \gamma^{\prime \prime}\right)(b c)^{2} \text {. }
$$

Dadurch entsteht:

$$
\begin{aligned}
& 0 \quad(\mu a)(\mu b)(\mu c)(\mu d)\left(\mu \gamma^{\prime}\right)\left(\mu \gamma^{\prime \prime}\right) \\
& \begin{array}{lllllll}
-\lambda^{3} & 0 & 0 & 0 & (a d) & 0 & 0
\end{array} \\
& \begin{array}{lllllll}
3 \lambda^{2} & 0 & 0 & (b c) & 0 & 0 & 0
\end{array} \\
& \Omega \equiv \begin{array}{ccccccc}
-3 \lambda & 0 & (b c) & 0 & 0 & 0 & 0 \\
1 & (a d) & 0 & 0 & 0 & 0 & 0
\end{array}, \\
& \begin{array}{lllllll}
-\rho \omega & 0 & 0 & 0 & 0 & 0 & \left(\gamma^{\prime} \gamma^{\prime \prime}\right)
\end{array} \\
& \begin{array}{lllllll}
0 & 0 & 0 & 0 & 0 & \left(\gamma^{\prime} \gamma^{\prime \prime}\right) & 0
\end{array}
\end{aligned}
$$

oder:

$\Omega=\left[\left(\mu \gamma^{\prime}\right)-\rho\left(\mu \gamma^{\prime \prime}\right)\right] \omega(b c)-\left(\gamma^{\prime} \gamma^{\prime \prime}\right)\left[\lambda^{3}(\mu d)+3 \lambda^{2}(\mu c)-3 \lambda(\mu b)-\left(\mu a^{\prime}\right]\right.$.

ES ist demnach:

$$
y_{i}=\left(\gamma_{i}^{\prime}-\gamma_{i}^{\prime \prime} \varrho\right) \omega(b c)-\left(\gamma^{\prime} \gamma^{\prime \prime}\right)\left[\lambda^{3} d_{i}+3 \lambda^{2} c_{i}-3 \lambda b_{i}-a_{i}\right] .
$$

Setzt man endlich:

$$
\lambda=\lambda_{1}{ }^{2},
$$


und demgemäss

$$
\omega=2 \lambda_{1}^{3} \sqrt{\frac{-2\left(\gamma^{\prime \prime} \gamma^{\prime \prime}\right)}{\rho(b c)}}=2 \lambda_{1}^{3} \Theta
$$

$$
y_{i}=\left(\gamma_{i}^{\prime}-\rho \gamma_{i}^{\prime \prime}\right) \Theta \lambda_{1}{ }^{3}-\left(\gamma_{1} \gamma_{1}\right)\left[\lambda_{1}{ }^{6} d_{i}+3 \lambda_{1}{ }^{4} c_{i}-3 \lambda_{1}{ }^{2} b_{i}-a_{i}\right]=\psi_{i} .
$$

Damit sind die sämmtlichen Tangenten derjenigen Haupttangentencurve, welche dem Parameter $\rho$ entspricht, als rationale Functionen sechsten Grades ausgedrückt. Zu jeder Erzeugenden der windschiefen Fläche gehören zwei Tangenten der Curve, diejenigen, welche den Werthen $\lambda_{1}$ und $-\lambda_{1}$ entsprechen. Man kann sich auch leicht davon überzeugen, dass die Function $\psi$ den folgenden Bedingungen:

$$
\left(\psi^{2}\right)=0, \quad\left(\frac{\partial \psi^{2}}{\partial \lambda}\right)=0, \quad(\varphi \psi)=0, \quad\left(\psi \frac{\partial \varphi}{\partial \lambda}\right)=0, \quad\left(\psi \frac{\partial^{2} \varphi}{\partial \lambda^{2}}\right)=0
$$

genügt. In der Gleichung (10) der developpabeln Fläche der Tangenten der Haupttangentencurve treten $\lambda_{1}^{5}$ und $\lambda_{1}$ nicht auf. Die Tangenten, welche den Werthen $\lambda_{1}=0, \lambda_{1}=\infty$ entsprechen, sind daher Rückkehrgeneratricen der developpabeln, d. h. stationäre Tangenten der Haupttangentencurve.

Die Haupttangentencurven der windschiefen Flächen dritter Ordnung sind daher rationale Curven vierter Ordnung und Classe sechsten Ranges mit zwei stationären Tangenten, welche mit den singulären Erzeugenden der Fläche coincidiren*).

Zugeordnet im Sinne des $\$$ XIII. sind der Developpabeln (10) unendlich viele Hlächen vierter Ordnung:

$$
\varrho z_{i}=\frac{\partial \psi_{i}}{\partial u_{1}}+k \frac{\partial \psi_{z}}{\partial \underline{u}_{1}}
$$

wo

$$
\begin{aligned}
& z_{i}^{\prime}=\frac{\partial \psi_{i}}{\partial \lambda_{1}}=-\left(\gamma^{\prime} \gamma^{\prime \prime}\right)\left[\lambda_{1}{ }^{4} d_{i}+2 \lambda_{1}^{2} c_{i}-b_{i}\right]+\left(\gamma_{i}^{\prime}-\gamma_{i}^{\prime \prime} \varrho\right) \lambda_{1} \Theta, \\
& z_{i}^{\prime \prime}=\frac{\partial \psi_{i}}{\partial \mu_{1}}=-\left(\gamma^{\prime} \gamma^{\prime \prime}\right)\left[\lambda_{1}{ }^{4} c_{i}-2 \lambda_{1}{ }^{2} b_{i}-a_{i}\right]+\left(\gamma_{i}^{\prime}-\gamma_{i}^{\prime \prime} \varrho\right) \lambda_{1}^{3} \Theta .
\end{aligned}
$$

Die beiden besonderen Flächen $z^{\prime} z^{\prime \prime}$ entstehen, wenn man durch jeden Punkt der Haupttangentencurve in der Schmiegungsebene derselben die beiden Geraden zieht, welche die beiden singulären Frzeugenden der windschiefen Fläche schneiden. Der Beweis dafür ist in den beiden Gleichungen

enthalten.

$$
\begin{aligned}
& \left(d z^{\prime}\right)=0, \\
& \left(a z^{\prime \prime}\right)=0
\end{aligned}
$$

*) Vgl. Clebseh, Ueber die Steiner'sche Fläche Crelle's Journal Bd. 67. Der rationale Charakter der Haupttangentencurven ist übrigens eine unmittelbare Folge davon, dass die Diseriminante $\sqrt{\Delta}=\sqrt{(\Psi \lambda \lambda)^{3}}$ (§ XII.) als Radical nur eine rationale Function zweiten.Grades, d, h. keine wesentliche Irrationalität exthält. 
Die Flächen $z$ bilden ein Büschel von windschiefen Flächen vier. ter Ordnung, welches eine sehr merkwürdige Beziehung zur Fläche dritter Ordnung besitzt. Jede dieser Elächen nämlich hat eire Doppelerzeugende, welche den Werthen

$$
\lambda_{1}= \pm \frac{1}{\sqrt{-k}}
$$

entspricht. Alle Doppelerzengenden dieser Art bilden eine Fläche, deren Erzeugende $t_{2}$ man erhält, wenn man in (11) an Stelle von $\lambda_{1}$ diesen Werth substituirt. $E_{s}$ ist daher

$$
\varrho t_{i}=d_{i}-k c_{i}+k^{2} b_{i}-k^{3} a_{i} \text {. }
$$

Die von den Doppelerzeugenden gebildete Fläche ist daher identisch mit der gegebenen windschiefen Fläche dritter Ordnung. Jede der Flächen $z$ gehört ferner unendlich vielen linearen Complexen an, welche zwei zusammenfallende Directricen besitzen. Die Coordinaten der letzteren sind, wie man leicht sieht:

$$
\rho u_{2}=d_{i}+h_{c_{i}}+k^{2} b_{\imath}+k^{3} a_{i} \text {. }
$$

Sie gehören also ebenfalls der nämlichen Fläche dritter Ordnung an. $t$ und $u$ sind conjugirte Erzeugende derselben, welche sich in einem Punkte der Doppelgeraden kreazen. Wir fassen diese Beperkungen in dem folgenden Satze zusammen:

Alle durch (11) dargestellten windschiefen Flächen vierter Ordnung besitzen also lauter algebraische Haupttangentencurven vienter Ordnung und Classe sechsten Ranges vom Geschlechte Null. Jede Fläche hat eine der Erzengenden der windschiefen Fläche dritter Ordnung zur Doppelgeneratrix und die its conjugirte zur Leitlinie, welche als Vereinigung von zwei Doppelgeraden aufzufassen ist. Für die beiden Flächen $k=0$, $k=\infty$ wird die Doppelgeneratrix zur Rückkehrgeneratrix.

Wir haben jetzt noch den Fall zu untersuchen, in welchem die windschiefe Fläche zusammenfallende singuläre Erzeugende besitzt. Unter der Voraussetzung

$$
4(b c)^{2}-\left(b^{2}\right)\left(c^{2}\right)=0
$$

fallen aber die beiden Geraden $\gamma^{\prime}, \gamma^{\prime \prime}$ ebenfalls zusammen. Die Fläche gehört also einem Büschel lineurer Complexe mit zwei wendlich nahen Leitlinien, von denen eine doppelt ist, an "Wählen wir den Parameter $\lambda$ so, dass $\lambda=0$ der singulären Erzeugenden entspricht, so ist zu setzen :

$$
\begin{gathered}
(a c)=0,(b c)=0,(c d)=0, \quad(a b)=0,\left(a^{2}\right)=0,\left(b^{2}\right)=0,\left(d^{2}\right)=0, \\
\left(c^{2}\right)+2(b d)=0 .
\end{gathered}
$$

Die Fläche gehört insbesondere dem speciellen Complexe mit der Axe $a$ an, welche gleichzeitig singuläre Erzeugende und Doppellinie 
ist. Die Gerade a mag die Axe der (Cayley'schen Linienfläche, genannt werden. Man hat ferner:

Daraus folgt:

$$
\begin{aligned}
& \rho a_{x}=0, \\
& \rho b_{x}=(b d) \lambda^{3}, \\
& \rho c_{x}=\left(c^{2}\right) \lambda^{2}, \\
& \rho d_{x}=(b d) \lambda .
\end{aligned}
$$

$$
4 b_{x} d_{x}-c_{x}^{2}=0 \text {. }
$$

Die sämmtlichen Erzeugenden der Fläche gehören also einem Complexe zweiten Grades an. Da derselbe ein specieller ist, so folgt weiter: Alle Erzeugenden der Fläche sind Tangenten eines Hyperboloides. Die Erzeugenden des letzteren sind dargestellt durch durch

$$
\underline{\rho} z_{i}=b_{i}+\lambda_{i}+d_{i} \lambda^{2} \text {. }
$$

Man erhält die Gleichung derjenigen developpabeln Flächen, welche zu Rückkehreurven die Haupttangentencurven der Fläche haben, wenn man nach den Vorschriften des \& XII. die $y$ als rationale Functionen von $\lambda$ ausdrückt. Indessen ist es überflüssig, diese Rechnung selbst durchzuführen. Die Theorie der abwickelbaren Flächen vierter Ordnung zeigt nämlich, dass die Gleichungen

$$
\varrho y_{i}=\frac{a_{i}}{4}+\frac{b_{i}}{3} \lambda+\frac{c_{i}}{2} \lambda^{2}+d_{i} \lambda^{3}+h_{i} \lambda^{1},
$$

in denen $k$ einen Parameter vorstellt, welcher den Bedingungen

$$
\left\{\begin{array}{c}
\left(k^{2}\right)=0, \quad(k d)=0, \quad(k c)=0, \quad(k b)=0, \\
3(k a)+(b d)=0
\end{array}\right.
$$

zu genügen hat, alle Haupttangenteneurven der Fläche repräsentiren. In der That kann man leicht verificiren, dass vermöge der Gleichungen (13) die Fläche (12) abwickelbar ist und jede ihrer Erzeugenden drei consecutive der Cayley'schen Fläche schneidet. Die Haupttangentenourven sind also vom vierten Range, dritter Ordnung und Classe, wie das auch aus den allgemeinen Formeln des $\S$ XII. herrorgehi, es sind Raumeurven dritter Ordnung*).

Zugeordnet sind ferner der Developpabeln (12) alle windschiefen Flächen

$$
z_{i}=\left(k a_{i}+\frac{b_{i}}{3}\right)+\left(k b_{i}+c_{i}\right) \lambda+\left(k c_{i}+3 d_{i}\right) \lambda^{2}+\left(k d_{i}+4 k_{i}\right) \lambda^{3}
$$

Da für jede derselben (12) eine Haupttangentencurve ist, so sind alle Flächen z ebenfalls Cayley'sche Linienflächen dritter Ordnung. Ihre Axen sind durch den Ausdruck

*) Vgl. die schon citirte Arbeit von Clebsch Crelle's Journal Bd. 67. 


$$
t_{i}=\frac{a_{i}}{4}+\frac{k_{i}}{3} k+\frac{c_{i}}{2} k^{2}+d_{i} k^{3}+k_{i} k^{2}
$$

gegeben, welcher mit der rechten Seite der Gleichungen (12) identisch ist. Man hat daher den folgenden Satz:

Die Haupttangentencwrven der Cayley'schen Fläche dritter Ordnung werden von den Axen zweifach unendlich vieler anderer Cayley'scher Flächen dritter Ordnung getildet, welche mit der gegebenen je eine Haupttangentencurve gemein haben.

Andere specielle windschiefe Flächen dritten Grades existiren nicht. Denn die Bedingung, dass die duxch den Ausdruck

$$
\varrho x_{i}=a_{i}+\lambda b_{i}+\lambda^{2} c_{i}+\lambda^{3} d_{i}
$$

dargestellte Fläche eine Doppelgeneratrix erhalte oder abwickelbar sel, führt auf das Verschwinden sämmtlicher Iuvarianten der Fundamentalcompiexe, d. h. auf Kegel oder ebene Curven.

\section{c. Windschiefe Flächen vierter Ordnung rom Geschlechte Null,}

Ihre Erzeugenden sind gegeben aureh die Gleichungen

$$
\varrho x_{i}=a_{i}+b_{i} \lambda+c_{i} \lambda^{2}+d_{i} \lambda^{3}+e_{i} \lambda^{4}
$$

unter Voraussetzung der Bedingungen:

$$
\left\{\begin{array}{cc}
\left(a^{2}\right)=0, \quad(b a)=0, & \left(e^{2}\right)=0, \quad(d c)=0, \\
2(a c)+\left(b^{2}\right)=0, & 2(c e)+\left(a^{2}\right)=0, \\
(a d)+(b c)=0, & (b e)+(c d)=0, \\
2(a c)+\left(c^{2}\right)+2(b d)=0 .
\end{array}\right.
$$

Dhe windschiefen Wlächen vierter Ordnung vom Creschlechte Null gehörex immer einem linearen Complexe $\gamma_{x}$ an, dessen Coefficienten $\gamma$ bestimmt sind durch die Gleichungen:

$$
(\gamma a)=0, \quad(\gamma b)=0, \quad(\gamma c)=0, \quad(\gamma d)=0, \quad(\gamma e)=0 .
$$

Sie enthalten daher in Allgemeinen eire algebraische Haupttangentencurve sechster Ordnung und Classe, zwölften Ranges mit zwölf stationären Tangenten vom Geschlechte Eins. Von den stationären Tangenten gehören vier den Cuspidalpunkten der Fläche an, während die anderen acht durch die Punkte der Eläche hindurchgehen, in denen ihre Erzeugenden von der Curve vierpunktiger Berührung berührt werden. Diese letatere ist vom achten Grade, viefundzwanzigsten Ranges vom Geschlechte Drei u. s. w.; sie kann zugleich angesehen werden als die vierfach zu zähleude Berührungscurve der Linienfläche der vierpunktigen Tangenten, deren Grad ebenfalls acht ist. Die Doppelcurre der Fläche ist die Raumeurve dritter Ordnung, welche acht Erzeugende berührt. Die drei Doppelebenen der Fläche, welche ron drei willkïhrlichen Punkten der Doppelcurve auslaufen, schneiden sich in demjenigen 
Punkte der durch jene drei bestimmten Ebene, welcher das Centrum des in der letzteren liegenden dem Complexe $\gamma$ angehörenden Büschels ist*). Jede Erzeugende bestimmt mit den vier Cuspidalpunkten der Fläche vier Ebenen, deren Doppelverbältniss dasselbe ist, wie das der vier Punkte, in denen sie selbst ron den parabolischen Ebenen der Fläche getroffen wird.

Die Parameter $\lambda_{1} \lambda_{2}$ zweier Erzeugenden $x, y$, welche sich in einem Punkte der Doppelcurve kreuzen, sind durch die Gleichung

oder:

$$
\Psi\left(\lambda_{1} \lambda_{2}\right)=0
$$

$$
\begin{array}{r}
(a c)+(a d)\left(\lambda_{1}+\lambda_{2}\right)+(a e)\left(\lambda_{1}+\lambda_{2}\right)^{2}+(b d) \lambda_{1} \lambda_{2}+(b e) \lambda_{1} \lambda_{2}\left(\lambda_{1}+\lambda_{2}\right) \\
+(c e) \lambda_{1}^{2} \lambda_{2}^{2}=0
\end{array}
$$

verbunden. Wir bezeichnen (15) als Gleichung der Doppelcurve. Die singulären Erzeugenden sind durch die Gleichung

(16) $\Psi \lambda \lambda=(a c)+2(a d) \lambda+4(a e) \lambda^{2}+2(b e) \lambda^{3}+(b d) \lambda^{2}+(c e) \lambda^{4}=0$

gegeben, deren linke Seite zugleich das Geschlecht der Haupttangentencurve charakterisirt. Man wird im Allgemeinen annehmen können, dass die Werthe $\lambda=0, \lambda=\infty$ sich auf singuläre Erzengende beziehen. Es treten dann zu den Gleichungen (14) noch die folgenden:

$$
\begin{cases}(a c)=0, & (c e)=0, \\ \left(b^{2}\right)=0, & \left(d^{2}\right)=0 .\end{cases}
$$

Es können aber von den singulären Erzeugenden auch einige oder alle zusammenfallen.

Die Erzengende $\lambda=0$ zählt für zwei singuläre, wenn

$$
\begin{array}{ll}
(a d)=0, & (a c)=0, \quad(c e)=0, \\
\left(b^{2}\right)=0, \quad(b c)=0, \quad\left(d^{2}\right)=0 .
\end{array}
$$

Treten zu diesen Gleichungen noch die folgenden

$$
(b e)=0,(c d)=0 \text {, }
$$

so gilt auch $\lambda=\infty$ für zwei singuläre Erzeugende.

Die Erzeugende $\lambda=0$ wird dagegen dreifich zu rechnen sein, wenn

$$
\begin{array}{ll}
(a c)=0, & (b d)=0, \quad 4(a e)+(b d)=0, \\
\left(b^{2}\right)=0, \quad(b c)=0, & 2\left(c^{2}\right)+(b d)=0 .
\end{array}
$$

Sie kann endlich vierfach zählen, wenn

$$
\begin{aligned}
& (a c)=0, \quad(a d)=0,4(a e)+(b d)=0, \quad(b e)=0, \\
& \left(b^{2}\right)=0, \quad(b c)=0,2\left(c^{2}\right)+(b d)=0, \quad(c d)=0 \text {. }
\end{aligned}
$$

*) Es ist dies nur ein specieller Fall eines allgemeineren Theorems, welches für jede einem linearen Complexe angehörige windschiefe Fläche gilt. 
In den letzteren Fällen ist die Haupttangentencurve immer vom Geschlechte Null. Der lineare Complex, welchem die llläche angehört, geht in keinem derselben in einen speciellen über. Eigentliche windschiefe Flächen der letzteren Art mit vier zusammenfallenden singulären Erzeugenden, welche einem speciellen linearen Complexe angehören, giebt es überhaupt nieht. Denn es wäre dann der letzten Reihe von Gleichungen noch $(a e)=0$ hinzuzufügen, womit zugleich die Fläche in einen Kegel oder in eine ebene Curve degenerirt. Wir werden daher ohne Beeinträchtigung der Allgemeinheit zwei discrete singuläre Erzeugende voraussetzen dürfen, denen die Parameter $\lambda=0, \lambda=\infty$ entsprechen.

An den Umstand, dass die Fläche immer einem linearen Cornplex angehört, kann man nun eine Classification derselben anknüpfen, welche, wie wir jetzt zeigen wollen, zu den pämlichen Resultaten führt, wie die Untersuchung, welche Herr Cremona über die verschiedenen Arten der windschiefen Flächen vierter Ordnung angestellt bat*).

In der That gehören alle Flächen vierter Ordnung $p=0$, für welche der lineare Complex ein allgemeiner ist, zur ersten Classe won Cremona. Die Fläche wird dagegen einem speciellen linearen Complex angehören, wenn die Determinante

$$
\begin{array}{lllll}
\left(a^{2}\right) & (a b) & (a c) & (a d) & (a e) \\
(a b) & \left(b^{2}\right) & (b c) & (b d) & (b e) \\
(a c) & (c b) & \left(c^{2}\right) & (c d) & (c e) \\
(a d) & (d b) & (d c) & \left(d^{2}\right) & (d e) \\
(a e) & (b e) & (e c) & (e d) & \left(e^{2}\right)
\end{array}
$$

verschwindet. Unter Berücksichtigung von (14) and (17) geht dieselbe über in:

$$
(b d)[(a d)(b e)-(a e)(b d)]\left[2(a d)(b e)+\left(c^{2}\right)(a e)\right] \text {. }
$$

Die Gleichung der Doppelcurve enthält die Grössen $\lambda_{1} \lambda_{2}$ nur in den Verbindungen $\lambda_{1}+\lambda_{2}, \lambda_{1} \lambda_{2}$. Sie wird daher in zwei lineare Factoren zerfallen, wenn ihre Determinante

oder

$$
\mid \begin{aligned}
& 2(a c)(a d) \quad(b d) \\
& (a d) 2(a e)(b e)=0, \\
& (b d)(b e) 2(e c) \mid
\end{aligned}
$$

ist.

$$
(b d)[(a d)(b e)-(a e)(b d)]=0
$$

*) Cremona, Sulle superficie gobbe di quarto ordine A. dell' accademia di Bologna t. VIII. 
Unter Voraussetzung von (19) zerfällt offenbar die Doppelcurve in zwei Gebilde niederer Ordnung. Die Gleichung (18) zeigt, dass die Fläche in diesem Falle immer eine Leitlinie, die Axe des Complexes $\gamma_{x}$ besitzt. Die Doppelcurve zerfällt daher in die Doppelgerade $\gamma$ und einen Doppelkegelschnitt. Es mag die Bemerkung hinzugefügt werden, dass die Doppelcurve sich auch in uiedere Gebilde auflösen kann, ohne dass die Gleichung (15) sich in lineare Factoren spaltet. Ihre Theile bestehen dann aber immer aus algebraisch gleichwerthigen Gebilden, d. h. aus einzelnen Doppelgeraden.

Wir betrachten zunächst den Fall $(b d)=0$. Die Gleichung der Doppelcurve ist dann

$$
\left(\lambda_{1}+\lambda_{2}\right)\left[(a d)+(a e)\left(\lambda_{1}+\lambda_{2}\right)+b e\left(\lambda_{1} \lambda_{2}\right)\right]=0 .
$$

Die Parameter einer Erzengenden, die sich in einem Punkte der Doppelcurve kreuzen, sind daher bestimmt durch die Gleichungen:

$$
\begin{gathered}
\lambda_{1}+\lambda_{2}=0 \\
(a d)+(a e)\left(\lambda_{1}+\lambda_{2}\right)+(b e) \lambda_{1} \lambda_{2}=0 .
\end{gathered}
$$

Von denselben bezieht sich die erstere auf die Doppelgerade der Fläche. Denn zwei Erzeugende $x^{\prime} x^{\prime \prime}$ mit den Parametern $\lambda$ und $-\lambda$ :

geben

$$
\begin{aligned}
& \varrho_{1} x_{i}^{\prime}=a_{i}+\lambda b_{i}+\lambda^{2} c_{i}+\lambda^{3} d_{i}+\lambda^{4} e_{i}, \\
& \varrho_{2} x_{i}^{\prime \prime}=a_{i}-\lambda b_{i}+\lambda^{2} c_{i}-\lambda^{3} d_{i}+\lambda^{4} e_{i}
\end{aligned}
$$

$$
\varrho_{1} x_{i}^{\prime}-\varrho_{2} x_{i}^{\prime \prime}=2 \lambda\left(b_{i}+\lambda^{2} d_{i}\right) \equiv b_{i}+\mu d_{i} .
$$

Die Fläche selbst ist auf vier gerade Linien $a b e d$ bezogen, von denen $a b, b d$, de sich in den Punkten $O, O^{\prime \prime}, O^{\prime}$ schneiden. Die Gleichung (20) zeigt, dass in jeder Doppelebene, welche zur Doppelcurve $\lambda_{1}+\lambda_{2}=0$ gehört, ein Strahl liegt, welcher dem Strahlbüschel $b d$ angehört, also durch den festen Punkt $O^{\prime \prime}$ geht. Diese Doppelebenen hüllen also einen Kegel zweiten Grades mit der Spitze $O^{\prime \prime}$ ein, eine Eigenschaft, welche die Doppelgerade charakterisirt. Die Gerade $0 O^{\prime}$ ist ferner die Axe des speciellen Complexes, dem die Fläche angehört, stellt also die Doppelgerade selbst dar. Sie ist zugleich die Axe der Doppelebenen, welche von den Punkten des Doppelkegelschnittes auslaufen. Die Punkte $O, O^{\prime}$ sind zwei Cuspidalpunkte der Fläche.

Die Ebene des Doppelkegelschnittes ist-bestimmt durch die beiden anderen Cuspidalpunkte, welche aus der Gleichung

$$
(a d)+2(a e) \lambda+(b e) \lambda^{2}=0
$$

gefunden werden, und den Punkt, in welchem derselbe die Doppelgerade $O O^{\prime}$ schneidet. Der letztere ist dadurch charakterisirt, dass ron ihm zwei Erzeugende auslaufen, deren Ebene durch die Doppel- 
gerade selbst geht. Man bestimmt die Parameter dieser beiden Frzeugenden, indem man in der Gleichung

$$
(a d)+(a e)\left(\lambda_{1}+\lambda_{2}\right)+(b e) \lambda_{1} \lambda_{2}=0
$$

$\lambda_{1}+\lambda_{2}=0$ setzt, und erhält

also

$$
\lambda^{2}=\frac{(a d)}{(b e)}
$$

Im zweiten Falle

$$
\lambda= \pm \sqrt{\frac{(a d)}{(b e)}}
$$

$$
(a d)(b e)-(a e)(b d)=0
$$

löst sich die Gleichung der Doppelcurve in die beiden Factoren

$$
\left\{\begin{aligned}
(a b)\left(\lambda_{1}+\lambda_{2}\right)+(b e) \lambda_{1} \lambda_{2} & =0 \\
(b d)+(b e)\left(\lambda_{1}+\lambda_{2}\right) & =0
\end{aligned}\right.
$$

auf. Der zweite Factor stellt wiederum die Doppelgerade vor. Bildet man nämlich den Ausdruck

$$
\begin{aligned}
\rho_{1} x_{i}^{\prime}-\rho_{2} x_{i}^{\prime \prime}=b_{i}+c_{i}\left(\lambda_{1}+\lambda_{2}\right) & +d_{i}\left(\left(\lambda_{1}+\lambda_{i}\right)^{2}-3 \lambda_{1} \lambda_{2}\right) \\
& +\sigma_{i}\left(\lambda_{1}+\lambda_{2}\right)\left(\left(\lambda_{1}+\lambda_{2}\right)^{2}-2 \lambda_{1} \lambda_{2}\right),
\end{aligned}
$$

so ergiebt sich sofort, dass rechterhand nur eine lineare Function von $\lambda_{1} \lambda_{2}$ vorhanden ist, da $\lambda_{1}+\lambda_{2}$ durch eine Constante ersetat werden kann (21). Man kann daher schreiben:

$$
\varrho_{1} x_{i}^{\prime}-\varrho_{2} x_{i}^{\prime \prime}=\alpha_{i}+\beta_{i} \lambda_{1} \lambda_{2} \text {. }
$$

Der Durchschnittspunkt der beiden Geraden $\alpha, \beta$ ist dalier wieder die Spitze eines Kegels, der von den Doppelebenen gebildet wird, die von der Doppelgeraden:

$$
(b d)+(b e)\left(\lambda_{1}+\lambda_{2}\right)=0
$$

auslaufen. Doppelgerade und Doppelkegelschnitt haben auch hier einen Punkt gemein, dessen Erzeugende durch das System von Werthen $\lambda_{1} \lambda_{2}$ dargestellt sind, welches die beiden Gleichungen (21) befriedigt.

Die Gleichung (19) charakterisint daher die Flächen der zweiten Classe von Cremona.

Wenn endlich in (18)

$$
2(a d)(b e)+\left(c^{2}\right)(a e)=0
$$

so gehört die Fläche einem speciellen linearen Complexe an, während die Doppelcurve nicht in ungleichartige Gebilde zerfällt. Es ist dann entweder eine einfache Leitlinie $\gamma$ vorhanden, und die Doppeleurve eine Raumcurve dritten Grades, während die Doppelebenen ein dreifaches Ebenenbüschel mit der Axe $\gamma$ bilden - Cremona VII oder die Leitlinie ist dreifach und die Doppeldeveloppable eine Fläche dritter Classe - Crem ona VIII - Beide Fälle stehen sich dualistisch in der folgenden Weise gegenüber. Im ersten liegen immer 
je drei Erzeugende in einer durch die Leitlinie gehenden Ebene, im zweiten gehen sie durch einen gemeinschaftlichen, auf jener Leitlinie liegenden Punkt.

Die Axe des speciellen Complexes kann endlich eine Erzeugende der Fläehe sein. Unter der Voraussetzung, dass $\lambda=0$ dieselbe darstelle, hat man dann die Bedingungen:

$$
\begin{array}{rll}
\left(a^{2}\right)=0, \quad(a b)=0, & (a c)=0, & (a d)=0, \quad(a e)=0, \\
\left(e^{2}\right)=0, \quad(d e)=0, & \left(b^{2}\right)=0, & (b c)=0, \\
2(b d)+\left(c^{2}\right)=0, & \left(d^{2}\right)=0, & (c e)=0 .
\end{array}
$$

Die Gleichung der Doppelcurve ist dann

$$
(b d)+(b e)\left(\lambda_{1}+\lambda_{2}\right)=0 \text {, }
$$

während zwei der singulären Erzeugenden mit der Erzeugenden $a$ coincidiren.

Je zwei Erzeugende, die sich in einem Punkte der Doppelcurve schneiden, liegen dann entweder mit $a$ in einer Ebene, die Doppeldeveloppable besteht aus dem dreifachen Ebenenbüschel $a$, oder je zwei Erzeugende dieser Art gehen durch einen Punkt ron $a$, die Doppelcurve besteht dann aus der dreifach zählenden Geraden $a$, während die Doppeldeveloppable ein Kegel zweiten Grades ist, zu welchem ein Ebenenbüschel durch a hinzu zu rechnen ist. Es sind dies die Fälle, welche bei Cremona dureh III und IV bezeichnet sind. Die windschiefen Flächen vierter Ordnung $p=0$ können auch unendlich vielen linearen Complexen angehören. Dazu ist erforderlich, dass einer der Coefficienten der Gleichung (14) eine lineare Combination der anderen vier ist. In allen diesen Fällen hat die Fläche zwei Leitgerade, alle Haupttangentencurven sind algebräische Curven, deren Tangenten sich im Allgemeinen rational durch elliptische Functionen ausdrücken lassen. Es ergeben sich nun die folgenden Fälle:

1) Die Fläche hat eine Doppel- oder Rückkehrgeneratrix. Um die Doppelgeneratrix in Evidenz treten zu lassen, nehmen wir an, dass die Parameter $\lambda=0, \lambda=\infty$ sich auf dieselbe beziehen. Die Gleichung der Fläche ist dann:

während:

$$
\varrho x_{i}=a_{i}+b_{i} \lambda+c_{i} \lambda^{2}+a_{i} \lambda^{3}+a_{i} \lambda^{4},
$$

$$
\begin{aligned}
\left(a^{2}\right)=0, \quad(a b)=0 & ,(a d)=0, \quad(a d)=0,(b c)=0, \\
& \left(b^{2}\right)+2(a c)=0, \\
& \left(c^{2}\right)+2(b d)=0, \\
& \left(d^{2}\right)+2(a c)=0 .
\end{aligned}
$$

Die beiden Leitlinien $\gamma^{\prime} \gamma^{\prime \prime}$, welche die Doppelcurve vertreten, sind bestimmt durch 


\section{(22)}

$$
\left(\gamma^{2}\right)=0, \quad(\gamma \alpha)=0, \quad(\gamma b)=0, \quad(\gamma c)=0, \quad(\gamma d)=0 .
$$

Beide sind discrete Doppellinien der Fläche, so lange die Determinante von (22) nicht verschwindet. Die fläche hat vier Cuspidalpunkte, bestimmt durch die Gleichung:

$$
\Psi \lambda \lambda=a c\left(1+\lambda^{1}\right)+(b d) \lambda^{2}=0 .
$$

Den Rang der Haupttangentencurven bestimmt man durch die Zahl ihrer Tangenten, welche eine willsührliche Gerade $z$ schneiden, d. h. indem man das Eliminationsresultat der Gleichungen

$$
\begin{gathered}
\left(y \frac{\partial^{2} \varphi}{\partial \lambda^{2}}\right)=0, \quad\left(y \frac{\partial^{2} \varphi}{\partial \lambda \partial \mu}\right)=0, \quad\left(y \frac{\partial^{2} \varphi}{\partial \mu^{2}}\right)=0, \\
(y z)=0, \quad y \gamma^{\prime}+\rho\left(y \gamma^{\prime \prime}\right)=0, \\
\left(y^{2}\right)=0
\end{gathered}
$$

bildet. Man erhält damit eine Controle für die Formeln des $\S$ XII., welche ebenfalls den Rang bestimmen. Es ergiebt sich so:

Die Hauptiangentencurven auf den windschiefon Flächen $p=0$ mit Doppelgeneratrix sind vom Geschlechte Eins, sechstex Ordnung und Classe, zwölften Runges, mit zwölf stationären Tangenten, won denen vier in die Cuspidalpurhte der Fläche fallen. Die Fläche hat daher vier hyperbolische Eraengende.

Die Parameter der letateren bestimrnt man auf tolgende Weise. Damit die Gleichungen:

$\left(y \frac{\partial^{3} \varphi}{\partial \lambda^{3}}\right)=0, \quad\left(y \frac{\hat{\partial}^{3} \varphi}{\partial \lambda^{2} \partial \mu}\right)=0, \quad\left(y \frac{\partial^{3} \varphi}{\partial \mu^{2} \partial \lambda}\right)=0, \quad\left(y \frac{\partial^{3} \varphi}{\partial \mu^{3}}\right)=0, \quad\left(y^{2}\right)=0$, oder

(23) $4 a_{y}+\lambda b_{y}=0, \quad 3 b_{y}+2 c_{y} \lambda=0, \quad 2 c_{y}+3 \lambda d_{y}=0, \quad d_{y}+4 a_{y} \lambda=0$ unendlich viele Werthe von $y$ zulassen, müssen die sechs Gleichungen:

$$
\mu_{i}\left(4 a_{i}+b_{\imath} \lambda\right)+\mu_{i}\left(3 b_{i}+2 c_{i} \lambda\right)+\mu_{3}\left(2 c_{i}+3 \lambda d_{i}\right)+\mu_{4}\left(d_{i}+4 a_{i} \lambda\right)=0
$$

bestehen können. Dies führt auf das Verschwinden einer Determinante von vier homogenen Gleichungen für die Coefficienten $\mu$, welche man erhält, wenn man die letzteren Gleichungen mit $a_{i} b_{i} c_{k} d_{i}$ multiplicirt and nach $i$ summirt, d. h. auf die Bedingung:

$$
\begin{aligned}
& \begin{array}{llll}
0 & \lambda & 1 & 0
\end{array} \\
& \begin{array}{llll}
\left(b^{2}\right) \lambda & 3\left(b^{2}\right) & 3(b d) \lambda & (b d)
\end{array} \\
& \begin{array}{lll}
4(a c) 2\left(c^{2}\right) \lambda & 2\left(c^{2}\right) & 4(a c) \lambda,
\end{array}=0 \text {, } \\
& (b d) \lambda \quad \beta(b d) \quad 3\left(d^{2}\right) \lambda \quad\left(d^{2}\right)
\end{aligned}
$$

welche reducirt die Gleichung

$$
\lambda^{1}=1
$$

liefert. Es ist übrigens von vornherein evident, dass die Gleichungen (23) für die Werthe 


$$
\lambda= \pm 1, \lambda= \pm i
$$

nur drei von einander wesentlich verschiedene darstellen. Diese Flächen bilden die fünfte Classe von Cremona.

Von den Flächen der fünften Cremona'schen Classe sind nicht wesentlich diejenigen verschieden, welche eine Rückkehrgeneratrix und zwei discrete Leitlinien besitzen. Aber in Ansehung der Haupttangentencurven und der hyperbolischen Erzeugenden zeigen dieselben ganz andere Eigenschaften, wie wir jetzt nachweisen werden. Form

Die Gleichung der Flächen mit Rückkehrgeneratrix $a$ kann in der

$$
\varrho x_{i}=a_{i}(1+\lambda)+c_{i} \lambda^{2}+d_{i} \lambda^{3}+e_{i} \lambda^{4}
$$

geschrieben werden, während

$$
\begin{aligned}
& \left(a^{2}\right)=0, \quad\left(e^{2}\right)=0, \quad(d e)=0, \quad(a c)=0, \quad(a d)=0, \\
& 2(c e)+\left(d^{2}\right)=0 \text {, } \\
& 2(a e)+\left(c^{2}\right)=0 \text {, } \\
& (a e)+(c d)=0, \quad 2(c d)=-2(a e)=\left(e^{2}\right) \text {. }
\end{aligned}
$$

Man kann zu diesen Bedingungen noch $\left(d^{2}\right)=0,(e c)=0$ hinzufügen. Die Fläche hat zwei singuläre Erzeugende, von deuen die eine dann dem Werthe $\lambda=\infty$ entspricht. Sie ist auf die drei Geraden $a, d, e$ bezogen, $d$ wird von $a$ und $e$ geschnitten. Durch den Cuspidalpunkt $e, d$ geht die eine Leitlinie $\gamma^{\prime}$, durch den Punkt ( $a d$ ) die andere. $\mathrm{Zu}$ den vierpunktigen Tangenten gehören zunächst die Linien, welche den drei Complexen

$$
a_{y}=0, \quad d_{y}=0, \quad c_{y}=0
$$

gemeinschaftlich sind. Dieselben bilden aber kein Hyperboloid, sondern zwei ebene Büschel, von denen das eine die Gerade $a \gamma^{\prime}$, das andere $a \gamma^{\prime \prime}$ enthält. Die Rückkehrgeneratrix ist daher keine eigentliche hyperbolische Erzeugende, vielmehr hat die Fläche nur die Erzetgende $\lambda=-4$ zur hyperbolischen.

Zur Untersuchung des Geschlechtes der Haupttangentencurven ist der Ausdruck $\Psi \lambda \lambda$ zu bilden. Da derselbe den Werth

$$
\text { (cd) } 4 \lambda^{2}(2 \lambda+1)
$$

annimmt, so sind die Haupttangentencurven rational.

Man erhält ferner als Rang derselben die Zahl 8. Sie sind daher rationale Curven fuinfter Ordnung und Classe achten Ranges, mit vier stationären Tangenten und sechs scheinbaren Doxpelpunlten.

Wir kehren jetzt wieder zu dem allgemeineren Falle einer Doppelgeneratrix zurück, Die beiden Doppelgeraden $\gamma^{\prime} \gamma^{\prime \prime}$ werden coincidiren, wenn die Determinante 
oder:

$$
\begin{array}{cccc}
0 & 0 & (a c) & 0 \\
0 & \left(b^{2}\right) & 0 & (b d) \\
(a c) & 0 & \left(c^{2}\right) & 0 \\
0 & (b d) & 0 & \left(d^{2}\right)
\end{array}
$$

$$
(a c)^{2}\left(\left(b^{2}\right)\left(d^{2}\right)-(b d)^{2}\right)
$$

verschwindet. Es sind demrach wieder zwei Fälle zu unterscheiden.

Wenn erstens

so folgt

$$
\left(b^{2}\right)\left(d^{2}\right)-(b d)^{2}=0
$$

$$
(b d)= \pm\left(b^{2}\right)
$$

Unter Voraussetzung des positiven Zejehens ist die Gleichung der Doppelcurve

$$
\left(\lambda_{1} \lambda_{2}-1\right)^{2}=0
$$

Die beiden Erzeugenden $x^{\prime} x^{\prime \prime}$, welche sich in einem Punkte derselben kreuzen, sind daher:

$$
\begin{aligned}
& \varrho_{1} x_{i}^{\prime}=a_{i}+b_{i} \lambda+c_{i} \lambda^{2}+d_{i} \lambda^{3}+a_{i} \lambda^{4}, \\
& \varrho_{1,} x_{i}^{\prime \prime}=a_{i} \lambda^{4}+b_{i} \lambda^{3}+c_{i} \lambda^{2}+a_{i} \lambda+a_{i} .
\end{aligned}
$$

Daraus ergiebt sich

$$
\varphi^{\prime} x_{i}^{\prime}-\varphi_{2} x_{i}^{\prime \prime}=b-d .
$$

Die Doppelebere enthält daher immer den festen Strahl $b-d$, welcher zugleich die allen linearen Complexen, denen die Fläche angehört, gemeinschaftliche Leitlinie bildet. Die Fläche besitzt daher die Doppelgeneratrix a zmd zwei dieselbe schneidende unendlich nahe Doppelgeraden $b-d$, welche immer mit den beiden sich auf ithen hreuzenden Erzengenden in einer Ebone liegen. Von den vier hyperbolischen Er. zeugenden

$$
\lambda= \pm 1, \lambda= \pm i
$$

sind die beiden ersten zu singulären Erzeugenden geworden. Die Haupttangentencurven sind daher rationale Curven vierter Ordmung und Classe, sechsten Ranges mit awei stationären Tangenten.

Diese Flächen bilden die sechste Classe bei Cremona, es sind dieselben, auf welche schon oben bei Betrachtung der Haupttangentencurven der windschiefen Fläche dritter Ordnung aufmerksam gemacht wurde.

Ist dagegen zweitens:

also

$$
(a c)=0
$$

$$
\left(b^{2}\right)=0,\left(d^{2}\right)=0,
$$

so coincidirt die Doppelgeneratrix mit den beiden unendlich nahen Leitlinien. Die Flache hat dann vier hyperbolische Eraengende, ihre 
Haupttangentencurven sind funfter Ordnung und Classe, achten Ranges mit vier stationären Tangenten (Cremona $X$ ).

Auch die Fläche mit Rückkehrgeneratrix kann unendlich nahe Leitlinien erhalten. Dabei treten zwei Fälle auf, die den eben behandelten analog sind. Im ersten vereinigt sich die hyperbolische Erzeugende mit der singulären, die Haupttangentencurven sind dann Raum. curven dritter Ordnung, im zweiten ist die Rückkehrgeneratrix mit den Leitlinien vereinigt und noch eine hyperbolische Erzeugende vorhanden, die Haupttangentencurven sind rationale Curven vierter Ordnung und Classe, sechsten Ranges mit zwei stationären Tangenten.

2) Die Fläche enthält heine Doppelerseugende. Von den beiden Leitlinien ist dann die eine dreifach, die andere einfach (Cremon a IX).

Wir erhalten demnach die folgende Uebersicht über die windschiefen Flächen vierter Ordnung vom Geschlechte Null:

A. Die Fläche gehört einem linearen Complexe an.

1) Der Complex ist allgemein Cremona $I$,

2) der Complex ist speciell.

a) Die Doppelcurve zerfällt in ungleichartige Gebilde, Cremona II.

b) Die Doppelcurve zerfallt nicht oder in gleichartige Gebilde, Cremona VII, VIII.

c) Die Axe des speciellen Complexes ist Erzengende der Fläche Cremana III, IV.

B. Die Fläche gehört unendlich vielen linearen Complexen an.

1) Das Complexbiischel hat discrete Directricen:

a) Flächen mit Doppel-oder Rückkehrgeneratrix, Cremona $V$,

b) Fläche ohne Doppelerzeugende, Cremona $I V$.

2) Das Complexbüschel hat zusammenfallende Directricen.

a) Fläche mit Doppel-oder Rïckiehrerseugenden, welche nicht mit den beiden unendlich nahen Leitlinien coincidirt, Cremona $V I$.

b) Fläche mit Doppel- oder Rückkehrgeneratrix, welche mit den beiden Leitlinien coincidirt, Cremona $X$.

Der Vollständigkeit halber sei hinzugefügt, dass die windschiefen Flächen vierter Ordnung rom Geschlechte Eins durch den Schnitt dreier Complexe erzeugt werden, von denen der erste zweiten Grades, die beiden anderen linear sind. Man hat daher zwei Classen dieser windschiefen Flächen zu unterscheiden, je nachdem die Directricen der beiden linearen Complexe verschieden sind oder zusammenfallen, wie dies auch Herr Cremon a gethan hat. Die Haupttangentencurven der ersten Classe sind algebraische Curven rom Geschlechte Acht, achter Ordnung und Classe, vierundzwanzigsten Ranges, mit vierzig stationären Tan- 
genten, von denen sich acht auf die singuläx'en, die übrigen zweiunddreissig auf die sechzehn hyperbolischen Erzeugenden der Fläche beziehen. Die Haupttangentencurven der zweiten Classe sind rom Geschlechte Eins, sechster Ordnung und Classe, zwölften Ranges rnit zwölf stationären Tangenten, von denen die zwölf hyperbolischen Erzeugenden der Fläche entsprechen.

Die abwickelbaren Flächen vierter Ordnung (rom Geschlechte Null) besitzen, da sie ebenfalls einem linearen Complexe angehören, eine in sich dualistische Rückkehrcurve. Die letztere kann daher nur die Raumcurve dritter Ordnung sein. Zugeordnet sind diesen Flächen Cayley'sche Linienflächen dritter Ordnung, welche die Rïckkehrcurve zur Haupttangentencurve haben. Diese Beziehung ist es, vermöge der man in den Stand gesetzt ist, den Ausdruck XIV, (12) für die Haupttangentencurven der Cayley'schen Fläche dritter Ordnung ohne weitere Rechnung hinzuschreiben.

Es ist ersichtlich, wie man in der bisher eingeschlagenen Richtung weiter gehen kaun. Die windschiefen Flächen fünfter Ordnung $p=0$ würden zunächst in drei Classen zerfallen, allgemeine, die einem linearen Complexe nicht angehören, zweitens Flächen eines linearen Complexes, endlich Flächen, die unendlich vielen Complexen angehören. Man übersieht ohne Weiteres, wie jeder dieser Fälle wieder eine Reihe besonderer einschliesst. Endlich treten noch abwickelbare Elächen auf, ihre Rückkehrcurven sind vierter Ordnung und Classe. Dieselben sind zugleieh Haupttangentencurven der ihnen zugeordneten Flächen vierten Grades, gehören aber nicht zur Classe der vorhin behandelten Haupttangentencurven, da die Fläche fünfter Ordnung einem linearen Complexe im Allgemeinen nicht angehört.

Göttingen, 6. F'ebruar 1874. 